\title{
THEORY OF THERMODYNAMIC MEASUREMENTS OF QUANTUM SYSTEMS FAR FROM EQUILIBRIUM
}

\author{
by
}

Abhay Shankar Chinivaranahalli Shastry

\author{
(B.) $\odot$ \\ A Dissertation Submitted to the Faculty of the \\ DEPARTMENT OF PHYSICS \\ In Partial Fulfillment of the Requirements \\ For the Degree of \\ DOCTOR OF PHILOSOPHY \\ In the Graduate College \\ THE UNIVERSITY OF ARIZONA
}




\section{THE UNIVERSITY OF ARIZONA GRADUATE COLLEGE}

As members of the Dissertation Committee, we certify that we have read the dissertation prepared by Abhay Shankar Chinivaranahalli Shastry

entitled Theory of thermodynamic measurements of quantum systems far from equilibrium

and recommend that it be accepted as fulfilling the dissertation requirement for the Degree of Doctor of Philosophy.

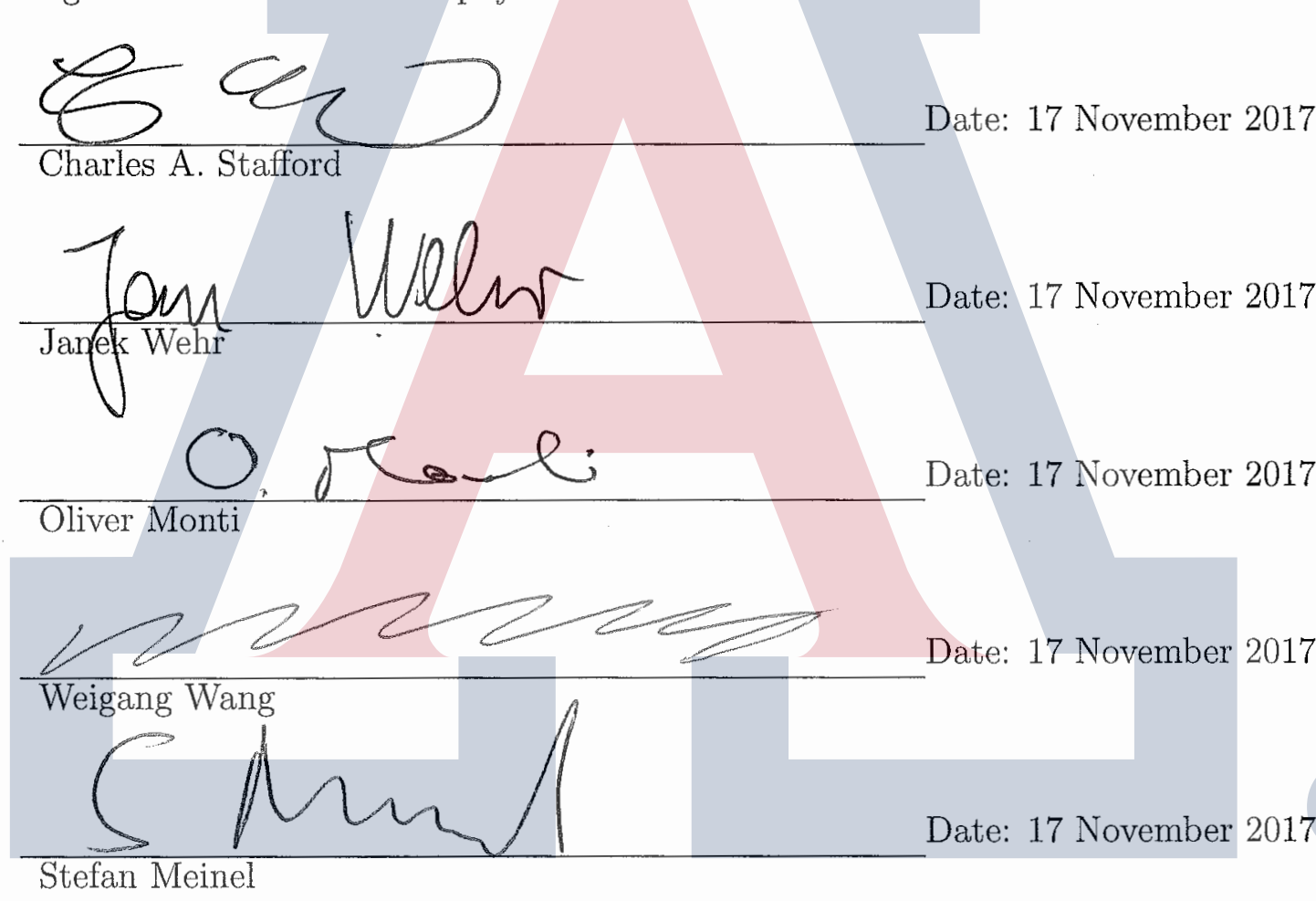

Final approval and acceptance of this dissertation is contingent upon the candidate's submission of the final copies of the dissertation to the Graduate College.

I hereby certify that I have read this dissertation prepared under my direction and recommend that it be accepted as fulfilling the dissertation requirement.
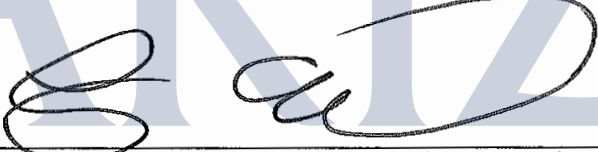

Dissertation Director: Charles A. Stafford

Date: 17 November 2017 


\section{STATEMENT BY AUTHOR}

This dissertation has been submitted in partial fulfillment of requirements for an advanced degree at the University of Arizona and is deposited in the University Library to be made available to borrowers under rules of the Library.

Brief quotations from this dissertation are allowable without special permission, provided that accurate acknowledgment of source is made. This work is licensed under the Creative Commons Attribution-No Derivative Works 3.0 United States License. To view a copy of this license, visit http://creativecommons.org/licenses/bynd/3.0/us// or send a letter to Creative Commons, 171 Second Street, Suite 300, San Francisco, California, 94105, USA.

SIGNED: Abhay Shankar Chinivaranahalli Shastry 


\section{ACKNOWLEDGEMENTS}

First and foremost, I am very grateful to my adviser Charles A. Stafford for his constant support and patient mentorship over the years. I have gained immensely from his in-depth knowledge of theoretical physics; I learned to critically examine scientific claims and hold my research to the highest objective standards. I will certainly miss our daily lunches and scientific discussions over coffee.

I'd like to thank my committee members Weigang Wang, Oliver Monti, Stefan Meinel and Janek Wehr for their careful reading of this thesis and their valuable feedback. I'd also like to thank Janek Wehr for his lectures in mathematical physics which helped me with parts of this work. I'm thankful also for the help I received from seniors in the group, Joshua Barr and Justin Bergfield, during my initial years. It's also been my great pleasure to work with enthusiastic undergraduate students Yiheng Xu, Marco Jimenez, Sosuke Inui and Marcus Rosales.

My parents, Sachidanand (Appa) and Kathyayini (Amma), have been incredibly supportive throughout my graduate studies and I am very grateful to them. Throughout my upbringing in India, my parents strived to afford me the unique luxury of freely pursuing my passion and have supported me constantly. I am also indebted to my sister Tejaswini who has always been concerned for my well-being.

I'd like to thank my colleagues Steve Steinke, Sarah Jones, Alex Abate, Matt Yankowitz, Pedro Espino, Rebekah Cross and Sophia Chen for their entertaining company and friendship; I'd especially like to thank Souratosh Khan and Adarsh Pyarelal for their steady friendship over the years. I'm grateful to Mike Strangstalein for his advice over the past year. Thanks to all the physics administrative staff, especially Bonnie Wood, Shane Smith, Gardie Lueders and Carmen Montijo, who have always been so eager to help; Thanks in particular to Sean Fleming (director of graduate studies) and Gardie, for the prompt help I received regarding my recent immigration-related matters. I'd also like to thank all my friends in the wider Tucson community who have made my stay so memorable!

I'm very grateful to all my teachers and friends from India. It's impossible to mention all the people who have helped me along the way in under one page. I'd like to thank my highschool friends Abhiram Muralidhar and Anandatheertha Suresh, my undergraduate friend Varun Narasimhachar, and my undergraduate adviser Tarun Deep Saini.

I am very grateful to my partner Shraddha Thumsi for her unwavering love and support over the past year.

Finally, I would like to thank the US Department of Energy for the financial support I received under award number DE-SC0006699. I believe the present thesis advances the scientific mission of the Department of Energy. 


\section{DEDICATION}

To my mother (Amma), who instilled in me scientific temper over dogmatism 


\section{TABLE OF CONTENTS}

LIST OF FIGURES . . . . . . . . . . . . . . . . . . . . . . . . . . . . . . . 9

LIST OF TABLES . . . . . . . . . . . . . . . . . . . . . . 15

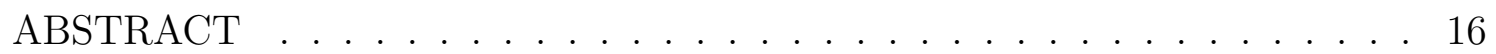

CHAPTER 1 Introduction . . . . . . . . . . . . . . . . . . . . . . . 17

CHAPTER 2 Temperature and Voltage . . . . . . . . . . . . . . . . . . 25

2.1 Introduction . . . . . . . . . . . . . . . . . . . . . 25

2.2 Expression for the Currents . . . . . . . . . . . . . . . . . . . 27

2.3 Local Measurements . . . . . . . . . . . . . . . . . . . . . . 30

2.3 .1 Example: Anthracene Junction . . . . . . . . . . . . . . . . . 32

2.3.2 Noninvasive Measurements . . . . . . . . . . . . . . . . . . . . 34

2.4 Uniqueness and the Second Law . . . . . . . . . . . . . . . . . . . . . . . . . . . 35

2.4 .1 Statements of the Second Law . . . . . . . . . . . . . . . . 36

2.4 .2 Uniqueness . . . . . . . . . . . . . . . . . . . . 43

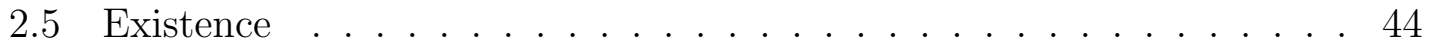

2.5 .1 Asymptotic Properties and Conditions for the Existence of a

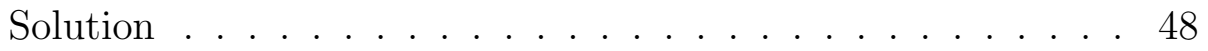

2.5.2 Ideal Probes: The Broadband Limit . . . . . . . . . . . . . . . 51

2.5.3 Nonunique Measurements: The Narrowband Limit. . . . . . . 53

2.5 .4 Example: Two-level System . . . . . . . . . . . . . . . 54

2.6 Conclusions . . . . . . . . . . . . . . . . . . . . . . . . . . 56

CHAPTER 3 Coldest Measurable Temperature . . . . . . . . . . . . . . . . 58

3.0 .1 Local Temperature and Voltage Measurements . . . . . . . . . 59

3.1 Local Temperatures near Absolute Zero . . . . . . . . . . . . . . . . . 62

3.1.1 Constant Trasmissions . . . . . . . . . . . . . . . . . . . . 64

3.1 .2 Transmission Node . . . . . . . . . . . . . . . . . . . . . 65

3.1.3 Higher-order Destructive Interference . . . . . . . . . . . . . . 66

3.1.4 Uniqueness and the Second Law . . . . . . . . . . . . . . . . . 68

3.2 Numerical Results and Discussion . . . . . . . . . . . . . . . . . . 69

3.2 .1 Local Temperatures . . . . . . . . . . . . . . . . . . . . . . 70

3.2 .2 Model of Probe-Sample Coupling . . . . . . . . . . . . . . . . 73

3.3 Conclusions . . . . . . . . . . . . . . . . . . . . . 73 


\section{TABLE OF CONTENTS - Continued}

CHAPTER 4 STM as a Thermometer . . . . . . . . . . . . . . . . . 78

4.1 Introduction . . . . . . . . . . . . . . . . . . . . . . . . . . 78

4.2 Temperature Measurements . . . . . . . . . . . . . . . . . . . 80

4.3 Temperature via Electrical Measurement . . . . . . . . . . . . . . . . 82

4.4 Results . . . . . . . . . . . . . . . . . . . . . . . . . . . . 87

4.4 .1 The System Hamiltonian . . . . . . . . . . . . . . . . . . . . 88

4.5 Conclusions . . . . . . . . . . . . . . . . . . . . . . 89

4.6 Note on Experimental Realization . . . . . . . . . . . . . . . . . . . . 89

CHAPTER 5 Entropy . . . . . . . . . . . . . . . . . . . . 91

5.1 Introduction . . . . . . . . . . . . . . . . . . . . . . . 91

$5.1 .1 \quad$ Single Fermionic Level . . . . . . . . . . . . . . . . . . . . . . 93

5.1 .2 Generalization to Many States . . . . . . . . . . . . . . . . . . 96

5.2 Entropy in the Scattering Basis . . . . . . . . . . . . . . . . . . . . 98

$5.2 .1 \quad$ Entropy Matrix of the System . . . . . . . . . . . . . . . . . . 100

$5.2 .2 \quad$ Entropy of Subspaces . . . . . . . . . . . . . . . . . . . 101

5.3 Entropy Inferred from Local Measurements . . . . . . . . . . . . . . . 103

5.4 Entropy Inferred from a Probe Measurement . . . . . . . . . . . . . . 104

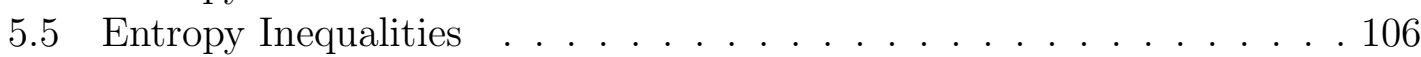

$5.6 \quad$ Entropy as a Metric for Local Equilibrium Departure . . . . . . . . . . 110

$5.6 .1 \quad$ Per-state Entropy Deficit . . . . . . . . . . . . . . . . . . 111

5.6 .2 Numerical Results . . . . . . . . . . . . . . . . . . . . . 112

5.7 Conclusions . . . . . . . . . . . . . . . . . . . . . 114

CHAPTER 6 Concluding Remarks . . . . . . . . . . . . . . . . 116

APPENDIX A The Nonequilibrium Steady State . . . . . . . . . . . . . . . . 119

A.0.1 Sum Rule for the Spectral Function . . . . . . . . . . . . . . . 120

A.0.2 Diagonality of $\hat{\rho}$. . . . . . . . . . . . . . . . . . . . 121

A.0.3 Positivity of $-i G^{<}(\omega)$ and $i G^{>}(\omega)$. . . . . . . . . . . . . . . . 122

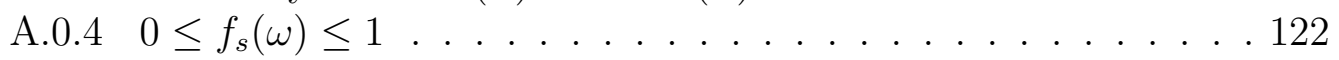

APPENDIX B Noninvasive Probes . . . . . . . . . . . . . . . . . . . . . . . 124

B.0.1 Dependence of $G$ on $\lambda$ and $x_{p} \ldots \ldots$. . . . . . . . . 125

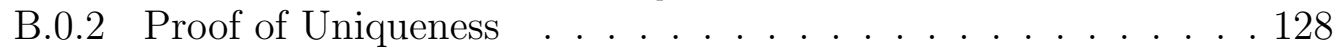

B.0.3 Proof of Existence . . . . . . . . . . . . . . . . . . . . . . . . 129

APPENDIX C Elastic Transport . . . . . . . . . . . . . . . . . . . . . 131

C.1 Linear Response Regime . . . . . . . . . . . . . . . . . . . . 133 
TABLE OF CONTENTS - Continued

REFERENCES . . . . . . . . . . . . . . . . . . . 135 


\section{LIST OF FIGURES}

1.1 (A) Schematic of the experimental setup showing the Pt resistance thermometer which measures the temperature of the probe tip. The resistance of the thermometer is in turn measured by applying a sinusoidal current $I_{f}$ and monitoring the voltage $V_{f}$. The electrical conductance is measured by the application of a small voltage across the single-atom junction and monitoring the resultant current. The thermal resistance network shown is used to calculate the thermal conductance of the junction by using the known value of the probe thermal resistance $R_{p}$. (B) Depicts the formation of the atomic contact junction. (C) and (D) show the SEM images of the scanning probe. Reproduced with permission from Cui et. al., Science, Vol. 355, Issue $6330(2017) . \quad \ldots \ldots \ldots \ldots 21$

2.1 Illustration of the measurement setup: The quantum conductor represented above is in a nonequilibrium steady state. A weakly-coupled scanning tunneling probe noninvasively measures the local voltage $\left(\mu_{p}\right)$ and local temperature $\left(T_{p}\right)$ simultaneously: By requiring both a vanishing net charge exchange $\left(I_{p}^{(0)}=0\right)$ and a vanishing net heat exchange $\left(I_{p}^{(1)}=0\right)$ with the system. The nonequilibrium steady state has been prepared, in this particular illustration, via the electrical and thermal bias of the strongly-coupled reservoirs (1 and 2). The measurement method itself is completely general and does not depend upon (a) how such a nonequilibrium steady-state is prepared, (b) how far from equilibrium the quantum electron system is driven, and (c) the nature of interactions within that system. $\quad . . . . . .31$

2.2 Upper panel shows the temperature and voltage variations along the molecular junction as seen by a probe scanning at a height of $3.5 \AA$. Lower panel shows the nonequilibrium distribution of the system $f_{s}$ and the equilibrium Fermi-Dirac distribution $f_{p}$ of the probe for two representative points marked 1 and 2 . The measurement finds $f_{p}$ so that the mean energy and occupancy of the probe is the same as that of the nonequilibrium system locally. The distribution $f_{s}$ at point 2 has the clear feature of mixing of two Fermi-Dirac distributions corresponding to the two reservoirs (shown with red and blue squares).

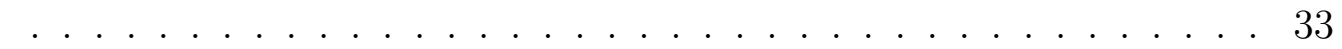




\section{LIST OF FIGURES - Continued}

2.3 Illustration of Lemma 1/ The contour $P Q$ shown in magenta cuts the number current contour $I_{p}^{(\mathrm{O})}=0$ (or any $I_{p}^{(\mathrm{O})}=$ constant) exactly once. The contour line from $P$ to $Q$ is at a constant temperature $\left(T_{p}=\right.$ constant $)$ and illustrates the Clausius statement: The number current is monotonically decreasing along $P Q$. The system and bias conditions are detailed in Sec. 2.5.4! . . . . . . . . . . . . . . . . . 37

2.4 Illustration of Lemma 2 : The contour $P Q$ shown in magenta cuts $I_{p}^{(1)}=0$ (or any $I_{p}^{(1)}=$ constant) exactly once. Contour $P Q$ is defined by $\mu_{p}=$ constant and illustrates the Clausius statement: The heat current is monotonically decresing along $P Q$. The system and bias conditions are detailed in Sec. $2.5 .4 !$. . . . . . . . . . . . . . . . . 40

\begin{tabular}{|ll|l|l|l}
\hline 2.5 & Left panel: Illustration of Theorem & 2 & for positive temperatures.
\end{tabular} The contour $P Q$ along $I_{p}^{(0)}=0$ (shown in blue) cuts the contour $I_{p}^{(1)}=0$ (shown in red) exactly once. Contour $P Q$ illustrates a certain statement of the second law of thermodynamics: The heat current is monotonically decreasing along $P Q$ (thus implying uniqueness). Right panel: The local spectrum sampled by the probe $\bar{A}(\omega)$ (black), the nonequilibrium distribution function $f_{s}(\omega)$ (red), and the probe Fermi-Dirac distribution $f_{p}(\omega)$ (blue) corresponding to the unique solution in the left panel. The resonances in the spectrum $A(\omega)$ correspond to the eigenstates of the closed two-level Hamiltonian (see Sec. 2.5.4) $\epsilon_{ \pm}= \pm 1$ shown in magenta. The Fermi-Dirac distribution is monotonically decreasing with energy, and corresponds to a situation with positive temperature (no net population inversion). The necessary and sufficient condition for the existence of a positive temperature solution is stated in Theorem $3:$. . . . . . . . . . . 45 


\section{LIST OF FIGURES - Continued}

2.6 Left panel: Illustration of Theorem 2 |for negative temperatures. The contour $P Q$ along $I_{p}^{(0)}=0$ (shown in blue) cuts the contour $I_{p}^{(I)}=0$ (shown in red) exactly once. Contour $P Q$ illustrates a certain statement of the second law of thermodynamics: The heat current is monotonically decreasing along $P Q$ (thus implying uniqueness). Right panel: The local spectrum sampled by the probe $A(\omega)$ (black, and nearly unchanged from Fig. 2.5 , , the nonequilibrium distribution function $f_{s}(\omega)$ (red), and the probe Fermi-Dirac distribution $f_{p}(\omega)$ (blue) which corresponds to the unique solution (shown in the left panel). The resonances in the spectrum $A(\omega)$ correspond to the eigenstates of the closed two-level Hamiltonian (see Sec. 2.5.4) $\epsilon_{ \pm}= \pm 1$ shown in magenta. The system has a net population inversion, satisfying the conditions of Corollary [3.1, and the probe Fermi-Dirac distribution is monotonically increasing with energy, corresponding

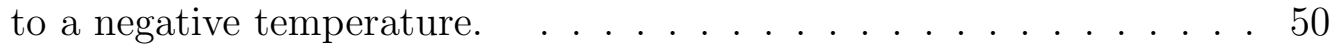

2.7 Schematic diagram of a two-level system coupled to two electron reservoirs under a voltage bias. (a) Bias condition not leading to population inversion. (b) Bias condition leading to population inversion due to direct injection into excited state. $\ldots \ldots \ldots \ldots 55$

3.1 Upper panels: Local temperature distributions for $\mathrm{Au}$-benzene- $\mathrm{Au}$ junctions in meta and para configurations, respectively. The thermal bias is supplied by cold $\left(T_{1}=0 \mathrm{~K}\right)$ and hot $\left(T_{2}=100 \mathrm{~K}\right)$ reservoirs covalently bonded to the atoms indicated by the blue and red squares, respectively. There is no electrical bias. The probe is scanned at a height of $3.5 \AA$ above the plane of the carbon nuclei in the molecule. The green dots shown in the temperature distributions correspond to the coldest temperature found in each of the junction configurations. Bottom panels: Transmission probabilities into the probe from $R 1$ (cold, i.e., blue curve) and $R 2$ (hot, i.e., red curve), when the probe is positioned over the coldest spot (shown by the corresponding green dot in the upper panel). A transmission node in the meta configuration leads to a greatly suppressed probe temperature [cf. table $3.1 \mid$. Note the very different vertical scales in the bottom panels. . . . . . . 75 


\section{LIST OF FIGURES - Continued}

3.2 Left panel: Probe temperature distribution in a Au-pyrene-Au junction under the same conditions described in Fig. 3.1. The green dot corresponds to the coldest temperature found by the search algorithm. Right panel: Transmissions into the probe from the hot reservoir $R 2$ (red) and the cold reservoir $R 1$ (blue) at the coldest position, indicated by the green dot on the left. The probe transmission from $R 2$ exhibits a (mid-gap) node at the Fermi energy $\mu_{0}$ of the reservoirs, thereby suppressing the temperature measured by the probe. . . . . 76

3.3 Upper panels: Probe temperature distributions for a Au-coronene$\mathrm{Au}$ junction under the same conditions described in Fig. 3.1 . The numerically calculated temperature is on the left, and to the right is the analytically calculated temperature using Eq. (3.24). Although it is in excellent qualitative agreement (and quantitative agreement for the most part), Eq. (3.24) poorly estimates the temperature for the coldest spot (shown in green) due to the existence of a transmission node. Eq. (3.31) gives the correct estimation in the presence of a node, while Eq. (3.24) incorrectly predicts $T_{p}=0 \mathrm{~K}$. The lower panel shows the probe transmissions from the two reservoirs $\left(R 1\right.$ with $T_{1}=0 \mathrm{~K}$ in blue and $R 2$ with $T_{2}=100 \mathrm{~K}$ in red) corresponding to the probe positioned over the coldest spot (shown in green). . . . . . . . . . . . 77

4.1 A schematic depiction of the measurement apparatus. The nanostructure (shown in green) is on an interconnect structure (shown in grey) which is connected to macroscopic reservoirs 1 and 2 which drives the system out of equilibrium using a voltage and/or temperature bias. The temperature and voltage must be simultaneously measured to ensure a unique measurement. The thermopower measurement uses a calibrated thermoresistor in series with the scanning tunneling tip which is in contact with the macroscopic probe reservoir p. Simultaneous measurement of the conductance and thermopower determines the temperature and voltage in the linear response regime. Measurements made in the tunneling regime result in a dramatic increase of the spatial resolution. . . . . . . . . . . . . . . 82 


\section{LIST OF FIGURES - Continued}

4.2 The conductance and thermopower, or equivalently the $\mathcal{L}_{p \alpha}^{(0)}$ and $\mathcal{L}_{p \alpha}^{(1)}$ coefficients, can be measured simultaneously by toggling between the two circuits above. The $\mathcal{L}_{p \alpha}^{(0)}$ coefficient is measured by applying a time-modulated voltage at the probe reservoir and recording the resulting current at the reservoir $\alpha$ while all other reservoirs are disconnected using switches, as shown on the left panel. $\mathcal{L}_{p 1}^{(0)}$ for example is measured with S1 ON and S2 OFF. The $\mathcal{L}_{p \alpha}^{(1)}$ coefficient is measured by inducing a time-modulated temperature variation in the probe and recording the current in reservoir $\alpha$ while all other reservoirs are disconnected using switches, as shown on the right panel. . . . . . . 85

4.3 Temperature variations on a hexagonal graphene flake with the application of a voltage bias $V_{\text {blue }}-V_{\text {red }}=5 \mathrm{mV}$, where the red and blue squares indicate the sites coupled to the interconnect. The left panel shows the exact linear response solution given in Eq. (4.5) while the right panel shows the approximate solution obtained by employing the WF law given in Eq. (4.6). The discrepancies between the two measurements are of higher order in the Sommerfeld series and are very small. In particular, $T_{p}^{(\mathrm{WF})}$ very slightly overestimates the hottest spots and underestimates the coldest spots while the intermediate temperatures agree exceptionally well. The same temperature scale is used for both panels. . . . . . . . . . . . . . . . . 86

4.4 Temperature variations on a hexagonal graphene flake with the application of a temperature bias $T_{\text {red }}-T_{\text {blue }}=30 \mathrm{~K}$, where the red and blue squares indicate the sites coupled to the interconnect. The left panel shows the exact linear response solution given in Eq. (4.5) while the right panel shows the WF solution given by Eq. (4.6). The discrepancies between the two measurements are of higher order in the Sommerfeld series and are very small. In particular, $T_{p}^{(\text {WF })}$ very slightly overestimates the hottest spots and underestimates the coldest spots while the intermediate temperatures agree exceptionally well. The same temperature scale is used for both panels. . . . . . . . . . . . . 87

$5.1 \quad$ The function $\sigma(f)=-f \ln f-(1-f) \ln (1-f)$. Illustration of its convexity: $\sigma\left(\lambda f_{1}+(1-\lambda) f_{2}\right) \geq \lambda \sigma\left(f_{1}\right)+(1-\lambda) \sigma\left(f_{2}\right) . \quad . . . . .106$

5.2 Subsystem entropy $S[\mathcal{A}]$ of a two-level quantum system far from equilibrium, plotted versus the inverse temperature $\beta=1 / T_{p}$ of the probe. The electrical bias across the system was varied from 1.6 to $3.2 \mathrm{~V}$. Values of $\beta<0$ correspond to absolute negative temperatures (population inversion). We see that $S[\mathcal{A}] \leq S_{s}[\mathcal{A}] \leq S_{p}[\mathcal{A}]$. . . . . . . . . 107 


\section{LIST OF FIGURES - Continued}

5.3 Upper panels (left to right): The local entropy-per-state of the system $s_{s}$, of the corresponding local equilibrium distribution $s_{p}$, and the local entropy deficit $\Delta s \equiv s_{p}-s_{s}$. The temperature distribution for the same junction (with identical bias conditions and sampling of probe positions) is shown in Fig. 3.2 , and we note that it resembles almost exactly the distribution $s_{p}$. Lower panels: The distributions $f_{s}$ and $f_{p}$ for three points shown in the upper panels, each having different probe temperatures $T_{p}=0.15 \mathrm{~K}, 15.9 \mathrm{~K}$ and $81.6 \mathrm{~K}$, respectively. The corresponding entropy deficits are $\Delta s=0.11,7.3$, and 1.5 , respectively, $\times 10^{-5}$. Point 2 , although closer to $0 \mathrm{~K}$ than point 3 is to $100 \mathrm{~K}$, is further from local equilibrium. . . . . . . . . . . 113 


\section{LIST OF TABLES}

3.1 The table shows the lowest temperatures found in the different junctions considered. All junctions have the same bias conditions: $T_{1}=0 \mathrm{~K}, T_{2}=100 \mathrm{~K}$ and no electrical bias. The right-most column shows the equation used to compute the temperature analytically. We obtain excellent agreement between the numerical and analytic results. The para configuration of the benzene junction does not display a node in the probe transmission spectrum and therefore the minimum probe temperature is not strongly suppressed. . . . . . . . 71

5.1 Table summarizing the notation and meaning of the different entropies based on local measurements. The entropies are inversely related to the information that is available to the local observer. Equilibrium probe entropy $S_{p}[\mathcal{A}]$ is the greatest of the three entropies and the observer in this case has the least amount of information regarding the system (colored in red). . . . . . . . . . . . . . 109 


\section{ABSTRACT}

Thermodynamics is a well-established field which studies systems in equilibrium and provides some of the most general results in all of physics. Unluckily, the vast majority of systems encountered in Nature are out of equilibrium. Thermodynamic descriptions of nonequilibrium systems are a formidable theoretical challenge and most results have been obtained under the assumption of a local equilibrium. Outside such an assumption, definitions of basic thermodynamic state variables such as temperature and voltage are muddled with a competing panoply of "operational" definitions. The work presented in this thesis provides a mathematically rigorous foundation for temperature and voltage measurements in quantum systems far from equilibrium. We show the existence and uniqueness of temperature and voltage measurements for any quantum fermion system in a steady-state, arbitrarily far from equilibrium, and with arbitrary interactions within the quantum system. We show that the uniqueness of these measurements is intimately tied to the second law of thermodynamics. In achieving this goal, we prove the positive-definiteness of the Onsager matrix in the context of thermoelectric transport which had only been a phenomenological statement for the past 85 years. The validity of the laws of thermodynamics far from equilibrium are discussed in detail. These results have fundamental implications for the field of scanning probe microscopy. We propose a method for imaging temperature fields in nanoscopic quantum conductors where we anticipate a remarkable improvement in the spatial resolution by over two orders of magnitude. Finally, we discuss the entropy of a quantum system far from equilibrium. We obtain a hierarchy of inequalities for the entropy of the quantum system and discuss its intimate relation to the information available from a measurement. We provide exact results pertaining to the entropy in the absence of many-body interactions but a working ansatz in their presence. 


\section{CHAPTER 1}

Introduction

The present thesis deals with several fundamental questions relating to the thermodynamic description of quantum systems that are far from equilibrium. Our primary question is whether it is meaningful to attribute, to a nonequilibrium system, values of temperature or voltage which effectively describe systems in thermodynamic equilibrium. The question of a temperature measurement outside equilibrium has been asked by various researchers in a variety of contexts, classical and quantum, and has generated a vast amount of scientific discussion but with no meaningful consensus [1]. The first order of business is then to understand the notion of equilibrium and the role of temperature and voltage in the equilibrium setting. We do this briefly here and then highlight the complications in extending notions of equilibrium thermodynamics to the nonequilibrium setting.

Equilibrium is an axiomatic assumption in statistical mechanics and thermodynamics. Two systems are said to be in a state of mutual equilibrium if there is no net exchange of energy and matter between them. Two systems which are not in mutual equilibrium will evolve towards a state of mutual equilibrium in a manner dictated by the second law of thermodynamics, that is, by maximizing the sum of their entropies. An isolated system therefore evolves in a manner where parts of the system (subsystems) are in mutual equilibrium with each other thereby increasing the total entropy. An equilibrium system is one in which there is no net flow of energy or matter between parts of the system. A system in a state of complete internal equilibrium has a spatially uniform temperature.

The zeroth law of thermodynamics introduces the notion of temperature as a parameter labeling thermal equilibrium states. The exitence of such equilibrium states is a central axiom of thermodynamics. Historically, the zeroth law of thermodynamics [2] appeared the latest but its importance lies in the pedagogical introduction 
of the notion of temperature. It states that thermal equilibrium states, labeled by their temperatures, form an equivalence class. That is, all systems labeled by the same temperature are in mutual thermal equilibrium. The zeroth law does not require the temperature to take on continuous values but only introduces temperature as a label (isotherm) for thermal equilibrium states. The equivalence property of thermal equilibrium states may be utilized to develop a thermometer: By choosing a system (thermometer) which is in mutual thermal equilibrium with the system of interest and having a readily measurable property which can be related directly to an empirical temperature. Properties which may be used to measure an empirical temperature are thermal expansion (e.g. of mercury), resistance of a metal, the thermopower of a thermocouple etc.

The intuitive concept of a "hotter" or "colder" temperature relies on the second law of thermodynamics. The Clausius statement of the second law of thermodynamics [3] establishes the direction of heat flow to be from the thermal equilibrium state at a hotter temperature to the one at colder one. A "hot" cup of coffee, for example, will cool down to reach the temperature of the room. The absolute temperature scale [4] which is used today was introduced by Thomson (Lord Kelvin) based on the Carnot's statement of the second law of thermodynamics. Thus, the notion of temperature draws from both the zeroth law and the second law of thermodynamics. Therefore, when defining a temperature for nonequilibrium systems, it becomes important to check its consistency with the laws of thermodynamics.

The thermodynamics of equilibrium systems [5] deals with thermodynamic equilibrium states and the transformation between such states. A majority of the discussion restricts these transformations to be reversible. A reversible transformation is one where the change in the system is brought about so slowly that at each instant of time the system may be assumed to be in a state of internal equilibrium. A reversible process does not change the entropy of the universe and at any stage the entropy gained by the system may be returned to the environment and vice-versa.

Early work on classical irreverible thermodynamics* considered situations where

${ }^{*}$ The classic textbooks by deGroot [6] or Prigogine [7] provide a detailed exposition. 
a local equilibrium could be assumed. In situations where such an assumption is valid, one may divide the system into smaller units with each such unit being close to equilibrium. The subunits are small enough to be considered homogenous and at equilibrium and large enough to warrant a macroscopic thermodynamic description. This leads to a major simplification and we may locally define temperature, voltage and other thermodynamic variables in the usual way as it is done for equilibrium systems. As suggested by the title of this thesis, we consider problems that are far from equilibrium where the local equilibrium hypothesis is not valid. We restrict our attention to quantum systems in a steady state.

The need for the development of a thermodynamic theory of quantum systems far from equilibrium is more pressing than ever owing to the remarkable progress in the design and fabrication of nanoscale devices. Commercial electronic devices have undergone a steady minituarization since the 1960s overtaking substantially Moore's initial prediction [8] that the trend would continue for a decade. The fabrication of mesoscopic devices in the 1980s has enhanced our understanding of electron transport at scales smaller than the mean free path of the electron where Ohm's law is no longer valid [9. The local equilibrium hypothesis would no longer be tenable at such length scales due to the absence of relaxation processes. This period saw the discovery of novel quantum phenomena such as conductance quantization [10], quantum Hall effect [11] and the experimental verification of the Aharanov-Bohm effect [12. The period concurrently saw the development of scanning probe techniques [13 16] which revolutionized the measurement of local electronic properties, providing remarkable spatial resolutions. The measurement of temperature and voltage were soon to follow with the development of scanning probe thermometry [17] and scanning probe potentiometry [18] respectively. The theoretical basis for such measurements, however, have remained unclear and the present thesis clarifies some of these foundational issues.

Recent technological developments have enabled physicists to observe charge and heat transport in devices whose dimensions are several orders of magnitude smaller than the electron mean free path. Single molecular junctions [19] and atomic contact 
junctions [20] represent the current frontier of minituarization of electronic devices. Experiments are now able to measure the conductance and thermopower of single molecular junctions [21] as well as atomic-size contact junctions [22]. While the quantization of electrical conductance in atomic contact junctions has been observed previously [23 25], recent years have seen a tremendous progress in the measurement of heat transport and dissipation at the atomic scale [26 28]. Fig. 1.1 shows the experimental setup used by Cui et. al. [27] to measure the heat current across a single-atom junction of gold and represents the current state-of-the-art in thermal measurements. Their setup is capable of measuring simultaneously the electrical and thermal conductances: For gold atomic contacts at room temperature, they find that the thermal conductance is quantized and follows the quantized electrical conductance. The insets (C) and (D) of fig. 1.1 show the scanning electron micrographs of their experimental setup. The temperature of the probe tip is measured by monitoring the change in the resistance of the Pt resistance thermometer shown in fig. 1.1 and the thermal resistance network shown in the inset (A) is used to calculate the thermal conductance.

The zeroth law of thermodynamics does not hold for systems outside equilibrium $\dagger^{\dagger}$ A thermometer records a temperature reading by reaching mutual equilibrium with the system of interest. In equilibrium, any two systems that have the same temperature reading will be in mutual equilibrium if brought into contact with each other. All degrees of freedom will have the same temperature for a system in a state of internal equilibrium. This would no longer be true outside equilibrium and the temperature that is measured would depend on the nature of interactions between the thermometer and the system. For example, a thermometer may couple strongly to some degrees of freedom while coupling weakly to others. There is no equipartition principle outside equilibrium and different degrees of freedom may have different temperatures. A thermometer may indeed measure the same temperature for two different systems that are out of equilibrium. However the two systems,

\footnotetext{
${ }^{\dagger}$ Ref. [1] provides a detailed exposition and reference list discussing the problems in extending the notion of temperature to nonequilibrium situations where no local equilibrium exists.
} 
Experimental setup and scanning electron microscopy (SEM) images of the scanning thermal probes.

\section{A}

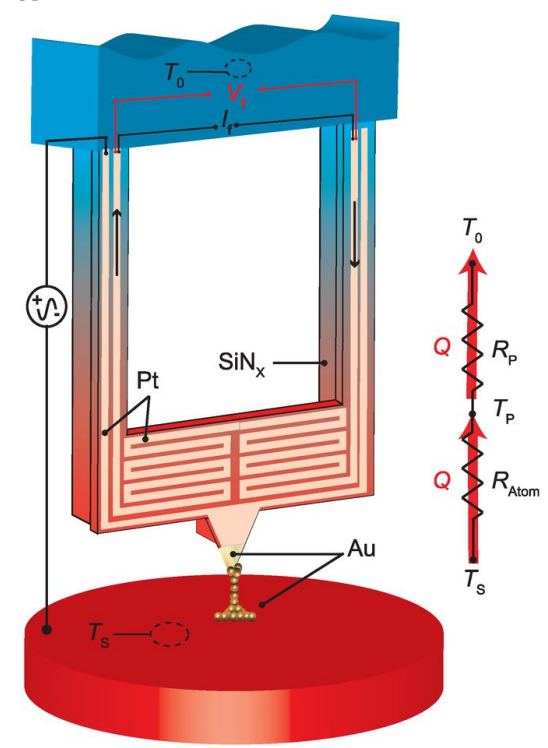

B
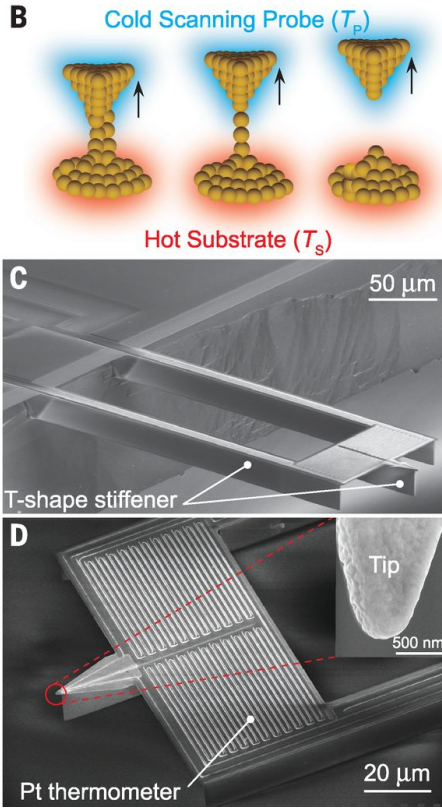

Longji Cui et al. Science 2017;355:1192-1195

Science

MIAAAS

Published by AAAS

Figure 1.1: (A) Schematic of the experimental setup showing the Pt resistance thermometer which measures the temperature of the probe tip. The resistance of the thermometer is in turn measured by applying a sinusoidal current $I_{f}$ and monitoring the voltage $V_{f}$. The electrical conductance is measured by the application of a small voltage across the single-atom junction and monitoring the resultant current. The thermal resistance network shown is used to calculate the thermal conductance of the junction by using the known value of the probe thermal resistance $R_{p}$. (B) Depicts the formation of the atomic contact junction. (C) and (D) show the SEM images of the scanning probe. Reproduced with permission from Cui et. al., Science, Vol. 355, Issue 6330 (2017). 
when brought into contact with each other, may not be in mutual equilibrium. The thermometer may couple strongly to some degrees of freedom in the first system while coupling strongly to others in the second system; there would be no reason to expect that the two systems would remain in mutual equilibrium when brought into contact with each other. We are well aware of this issue and we explicitly consider the electronic degrees of freedom in all the results presented in this thesis. However, the results are equally applicable to any fermionic degree of freedom so long as it is understood that one is talking about the temperature and voltage corresponding to those fermions alone.

The invalidity of the zeroth law outside equilibrium has prompted some authors to regard definitions of temperature or voltage as "operational" and lacking any fundamental meaning. For example, McLennan states in his book [29] "Clearly there is no zeroth law for a nonequilibrium situation. The reading of a thermometer may depend on its orientation, shape, surface properties and so on ... Nonequilibrium temperature is introduced for theoretical convenience rather than to take advantage of a basic principle." We disagree with this point of view. The question of the zeroth law of thermodynamics was analyzed by Stafford et. al. in earlier works [30, 31] in some detail. The present thesis concerns itself mainly with the second law of thermodynamics. The results presented in this thesis take the notions of temperature and voltage well beyond their "operational" role in describing nonequilibrium systems.

One of the main results presented in this thesis is the proof of the Onsager's statement of the second law of thermodynamics. We shall see that this result provides a rigorous mathematical foundation for the notions of temperature and voltage in the nonequilibrium setting. Onsager's seminal work of 1931 [32, 33] introduced the so-called linear response coefficients and derived the reciprocal relations that they satisfy. Onsager's contribution was an influential starting point for the later development of nonequilibrium thermodynamics where a local equilibrium hypothesis was assumed. The results presented in the present thesis pertain to quantum fermion systems arbitrarily far from equilibrium. The theoretical basis for the description of 
quantum systems out of equilibrium traces back to the pioneering work of Martin, Schwinger [34, 35], Kadanoff, Baym [36], Keldysh [37] and others in the 1960s. This formalism is now referred to in literature as the Nonequilibrium Green's Functions (NEGF) formalism and sometimes as the Keldysh formalism. The formalism was applied to the study of electron transport through an interacting nanostructure (e.g. Ref. [38]) during the 1990s. We do not review the NEGF formalism in the present thesis but include some useful results in the appendices. A pedagogical introduction to the subject can be found in the book by Stefanucci and van Leeuwan [39]. A brief overview of the Green's function formalism relevant to quantum steady-state transport can be found in the $\mathrm{PhD}$ thesis of Joshua Barr [40].

The present thesis is organized as follows. In chapter 2, we present results which place temperature and voltage on a rigorous mathematical foundation. We show that the uniqueness of the temperature and voltage measurement is a consequence of the second law of thermodynamics. Interestingly, we find that temperature and voltage have to be defined simultaneously to ensure uniqueness. We show also that a solution always exists. These results are very general: They apply to any quantum fermion system in a steady-state, arbitrarily far from equilibrium, with arbitrary many-body interactions within the quantum system. We also show that one may obtain negative temperatures if the quantum system has a spectrum that is bounded above. Chapter 3 considers a question motivated by the third law of thermodynamics: What is the coldest possible temperature for a nonequilibrium quantum system? In chapter 4, we present a novel experimental method for temperature measurements using a scanning tunneling microscope whose working principle is closely related to the findings of the preceding chapters. We anticipate a remarkable improvement in the spatial resolution of temperature measurements by two orders of magnitude over existing schemes of scanning thermal microscopy. Any theory of thermodynamics is incomplete without a thorough exploration of the notion of entropy. In chapter 5, we discuss the entropy of a steady-state quantum system and obtain a hierarchy of inequalities. The entropy inequalities that we obtain have an intimate relationship with the information available to an observer from a local measure- 
ment. The analysis is done here in the scattering basis and we present rigorous results for the noninteracting case. Including interactions will cause the scattering states to mix and the analysis becomes considerably more complicated. We may straight-forwardly extend the definition to apply for such a case but, since a rigorous justification is missing, we would like to call it an ansatz when interactions are present. We also discuss the use of entropy as a metric to quantify the departure from equilibrium. Some useful results pertaining to the Green's functions are included in the appendices. In order to benefit the reader, we have included brief introductions at the start of every chapter and the reader may choose to skip to a chapter of particular interest, hopefully, without loss of context团.

\footnotetext{
${ }^{\ddagger}$ The analysis in chapter 3 follows from the more general results presented in chapter 2 and we do not include a separate introduction.
} 


\title{
CHAPTER 2
}

\author{
Temperature and Voltage
}

This chapter is based on the work published in Ref. [41]

"The law that entropy always increases holds, I think, the supreme position among the laws of Nature. If someone points out to you that your pet theory of the universe is in disagreement with Maxwell's equations - then so much the worse for Maxwell's equations. If it is found to be contradicted by observation - well, these experimentalists do bungle things sometimes. But if your theory is found to be against the second law of thermodynamics I can give you no hope; there is nothing for it but to collapse in deepest humiliation." - Arthur Eddington [42].

\subsection{Introduction}

Temperature and voltag $\oplus^{*}$ are basic thermodynamic quantities which are routinely measured in all sorts of experiments. The definition of temperature and voltage are well established in equilibrium. The zeroth law encodes the definition of temperature as an equivalence class of equilibrium systems where any two systems from the same class, when brought into contact, do not have a net exchange of energy. The second law tells us that the energy (in the form of heat) flows from a system at a higher temperature to a system at lower temperature in the absence of work. Similarly, particles flow from a system at higher chemical potential to a system at lower chemical potential in the absence of temperature gradients. Here we show that such formulations of the second law are indeed possible for quantum systems far from equilibrium and that they have profound consequences for the measurement of

\footnotetext{
*Voltage here refers to the electrochemical potential $(\mu)$ and not just the electrostatic potential $(\mathrm{V})$. A voltmeter in fact measures the electrochemical potential difference which of course includes the electrostatic contribution $\mu=\mu_{0}+e \mathrm{~V}$.
} 
temperature and chemical potential (or voltage when dealing with charged particles). Just as Eddington notes, our statements of the second law are not dependent on the nature of interactions within the quantum system.

The definition of thermodynamic variables in a system far from equilibrium is of fundamental interest. It serves as a necessary step towards the construction of nonequilibrium thermodynamics which has gained renewed interest in recent years [1, 31, 43 48]. On the experimental side, scanning tunneling potentiometry [18] (i.e., the measurement of local voltages) has undergone tremendous progress over the past two decades [49 51] achieving sub-angstrom spatial resolutions. Scanning thermometry [17] has proven significantly more challenging but is currently undergoing a rapid evolution toward nanometer resolution [52 55]. The progress on the theoretical side is therefore ever more important since predictions will soon be experimentally testable. Unfortunately, even the notions of temperature and voltage out of equilibrium have not been thought out carefully. Until now, mainly operational definitions [46, 56, 65] have been advanced leading to a competing panoply of often contradictory definitions of such basic observables as temperature and voltage. The results in this chapter fill this gap by developing a rigorous mathematical description of local thermodynamic measurements.

One other point deserves mention before we look at the results of this chapter. Out of equilibrium, different degrees of freedoms can have different temperatures. We know this fact from NMR spectroscopy where the nuclear spin temperature can be negative but the atom or electron temperature is clearly positive. Another example is that of a laser, where electron temperature is negative due to population inversion but the lattice/gas temperature is positive. We therefore have to clearly demarcate between lattice temperature [66 68], photon temperature [69-71] and electron temperature [30, 31, 46, 56, 59, 61, 63, 64]. Our work concerns itself only with the electron temperature. The results presented in this chapter are, however, true for any fermionic system, charged or neutral.

The main results of this chapter are presented in the lemmas and theorems in the subsequent sections. The brief summary of these results is as follows. We 
show that temperature and voltage cannot be measured separately for a system out of equilibrium. This is shown in Lemmas 1, 2 and Theorems 1 and 2, Lemma 11 shows that a voltage-only measurement is nonunique and hence voltage is illdefined without an accompanying temperature measurement. Lemma 2 shows that a temperature-only measurement is nonunique and hence temperature is ill-defined without an accompanying voltage measurement. Theorem 1 is in fact the Onsager statement of the second law which, to our knowledge, is proved for the first time. Lemmas 1 and 2 are also statements of the second law related to the Clausius statement. Theorem 2 shows that the simultaneous measurement is unique and hence the only meaningful way to define temperature and voltage. Theorem 3 answers the question of the existence of a solution. Theorem 3 and its corollary 3.1 establish that a solution always exists. There is also the question of an ideal probe which is addressed extensively.

The results here are quite general in their applicability: They hold for steadystate quantum systems arbitrarily far from equilibrium, with arbitrary interactions

within the quantum system, and for any fermionic system, charged or neutral. One important consideration is that of a noninvasive probe. In the presence of interactions, our results only hold for the noninvasive probe limit. Analysis outside this limit appears to be mathematically complicated. If interactions are absent, then the noninvasive probe limit is not needed and the results hold for arbitrarily strong system-probe couplings. This latter point is explicitly shown in appendix B, section B.0.2.

\subsection{Expression for the Currents}

We use the nonequilibrium Green's function formalism (NEGF) for describing the motion of electrons within a quantum conductor. A general expression for the nonequilibrium steady-state electrical current $(\nu=0)$ [38 and the electronic contribution to the heat current $(\nu=1)$ [72] flowing into a macroscopic electron reservoir 
$P$ is

$$
I_{p}^{(\nu)}=-\frac{i}{h} \int_{-\infty}^{\infty} d \omega\left(\omega-\mu_{p}\right)^{\nu} \operatorname{Tr}\left\{\Gamma^{p}(\omega)\left(G^{<}(\omega)+f_{p}(\omega)\left[G^{>}(\omega)-G^{<}(\omega)\right]\right)\right\},
$$

where $\Gamma^{p}(\omega)$ is the tunneling width matrix describing the coupling of the probe to the system and

$$
f_{p}(\omega)=\left\{1+\exp \left[\left(\omega-\mu_{p}\right) / k_{B} T_{p}\right]\right\}^{-1}
$$

is the Fermi-Dirac distribution of the probe. $G^{<}(\omega)$ and $G^{>}(\omega)$ are the Fourier transforms of the Keldysh "lesser" and "greater" Green's functions [39], describing the nonequilibrium electron and hole distributions within the system, respectively.

Eq. (2.1) is a general result valid for arbitrary interactions within the quantum system. It is also valid for situations arbitrarily far from equilibrium. A lot of insight can be gained by a rearrangement of Eq. (2.1) into the Landauer-Büttiker form [31]

$$
I_{p}^{(\nu)}=\frac{1}{h} \int_{-\infty}^{\infty} d \omega\left(\omega-\mu_{p}\right)^{\nu} \mathcal{T}_{p s}(\omega)\left[f_{s}(\omega)-f_{p}(\omega)\right] .
$$

The Landauer-Büttiker form was originally derived for noninteracting electrons within the scattering approach therefore it is crucial to note that Eq. (2.2) is valid for arbitrary interactions. It is simply a rearragement of Eq. 2.1).

Eq. (2.2) is describing the charge and heat exchanged by the probe reservoir $p$ (which is approximated to be at equilibrium) with a nonequilibrium system $s$. We show that $\mathcal{T}_{p s}(\omega)$ satisfies the property of a transmission function and is nonnegative. We show also that $f_{s}(\omega)$ satisfies the property of a fermionic distribution function and takes on values in the range $[0,1]$.

We first write down the expression for $f_{s}$ and $\mathcal{T}_{p s}$ in terms of the Green's functions. We refer to $f_{s}$ as the local nonequilibrium distribution function of the system, as sampled by the probe 31]

$$
f_{s}(\omega) \equiv \frac{\operatorname{Tr}\left\{\Gamma^{p}(\omega) G^{<}(\omega)\right\}}{2 \pi i \operatorname{Tr}\left\{\Gamma^{p}(\omega) A(\omega)\right\}},
$$

and the effective probe-system transmission function

$$
\mathcal{T}_{p s}(\omega)=2 \pi \operatorname{Tr}\left\{\Gamma^{p}(\omega) A(\omega)\right\} .
$$


$A(\omega)$ is the spectral function given by

$$
A(\omega)=\frac{1}{2 \pi i}\left(G^{<}(\omega)-G^{>}(\omega)\right),
$$

and is Hermitian and positive-semidefinite as shown in appendix $\mathrm{A}$.

Since the probe-system coupling $\Gamma^{p}(\omega)$ is also positive-semidefinite (due to causality [39]), we note that

$$
\begin{aligned}
\operatorname{Tr}\{A(\omega) \Gamma(\omega)\} & =\operatorname{Tr}\left\{A(\omega)^{1 / 2} A^{1 / 2}(\omega) \Gamma(\omega)\right\} \\
& =\operatorname{Tr}\left\{A^{1 / 2}(\omega) \Gamma(\omega) A^{1 / 2}(\omega)\right\} \\
& \geq 0
\end{aligned}
$$

where $A^{1 / 2}(\omega)$ is the positive-semidefinite square root of $A(\omega) \cdot A^{1 / 2}(\omega) \Gamma(\omega) A^{1 / 2}(\omega)$ becomes positive-semidefinite when $A^{1 / 2}(\omega)$ and $\Gamma(\omega)$ are positive-semidefinite [73] and therefore we have

$$
\mathcal{T}_{p s}(\omega) \geq 0, \forall \omega \in \mathbb{R} .
$$

We also show in appendix $\mathrm{A}$ that $f_{s}$ satisfies the property of a fermionic distribution function:

$$
0 \leq f_{s}(\omega) \leq 1 \quad \forall \omega \in \mathbb{R} .
$$

We start our analysis with the following postulate and explain its physical significance.

Postulate 1 The local probe-system transmission function $\mathcal{T}_{p s}: \mathbb{R} \rightarrow[0, \infty)$ and the nonequilibrium distribution function $f_{s}: \mathbb{R} \rightarrow[0,1]$ are measurable over any interval $[a, b] \in \mathbb{R}$ and $\mathcal{T}_{p s}(\omega)$ satisfies

$$
0<\int_{-\infty}^{\infty} d \omega \mathcal{T}_{p s}(\omega)<\infty
$$

and

$$
\left|\int_{-\infty}^{\infty} d \omega \omega \mathcal{T}_{p s}(\omega)\right|<\infty .
$$

The measurability of $\mathcal{T}_{p s}(\omega)$ and $f_{s}(\omega)$ is taken to lend meaning to the currents in Eq. (2.2). We point out that the finiteness of the two integrals given in Eqs. (2.9) and (2.10) is more relevant to our discussion of existence of solutions in Sec. 
2.5. Our result on uniqueness, as stated in Theorem 2, is somewhat stronger and requires only that the function $\mathcal{T}_{p s}(\omega)$ grow slower than exponentially for large values of energy (for $\omega \rightarrow \pm \infty$ ).

On physical grounds, the probe-sample transmission function $\mathcal{T}_{p s}(\omega)$ can be argued to have a compact support (non-zero only for some finite interval $\left[\omega_{-}, \omega_{+}\right] \subset \mathbb{R}$ ). It is easy to see that $\mathcal{T}_{p s}$ must have a lower bound $\omega_{-}$such that $\mathcal{T}_{p s}(\omega)=0 \forall \omega<\omega_{-}$, since physical Hamiltonians must have a finite ground-state energy. However, for energies larger than the probe work function $\left(\omega_{+}\right)$, it can be argued that the particle will merely pass through the probe and not contribute to the steady state currents into the probe. $\mathcal{T}_{p s}(\omega)$ then has a compact support and satisfies Eqs. (2.9) and (2.10). In section 2.5, we comment upon the limiting case where the measure of $\omega \mathcal{T}_{p s}(\omega)$ in Eq. (2.10) tends to infinity. The absolute value on the lhs in Eq. (2.10) is somewhat redundant since the limiting case must have $\omega_{+} \rightarrow \infty$ while $\omega_{-} \rightarrow-\infty$ is ruled out based on the principle that any physical spectrum has a finite ground-state energy. We note that Eqs. 2.9), 2.10) also imply

$$
0<\int_{-\infty}^{\infty} d \omega \mathcal{T}_{p s}(\omega) f_{s}(\omega), \int_{-\infty}^{\infty} d \omega \mathcal{T}_{p s}(\omega) f_{p}(\omega)<\infty
$$

and

$$
\int_{-\infty}^{\infty} d \omega \omega \mathcal{T}_{p s}(\omega) f_{s}(\omega), \int_{-\infty}^{\infty} d \omega \omega \mathcal{T}_{p s}(\omega) f_{p}(\omega)<\infty
$$

\subsection{Local Measurements}

The local voltage and temperature of a nonequilibrium quantum system, as measured by a scanning thermoelectric probe, is defined by the simultaneous conditions of vanishing net charge dissipation and vanishing net heat dissipation into the probe [30, 31, 48, 63, 64, 74]:

$$
I_{p}^{(\nu)}=0, \quad \nu \in\{0,1\},
$$

where $\nu=0,1$ correspond to the electron number current and the electronic contribution to the heat current, respectively. Eq. (2.13) gives the conditions under which the probe is in local equilibrium with the sample, which is itself arbitrarily far from equilibrium. 


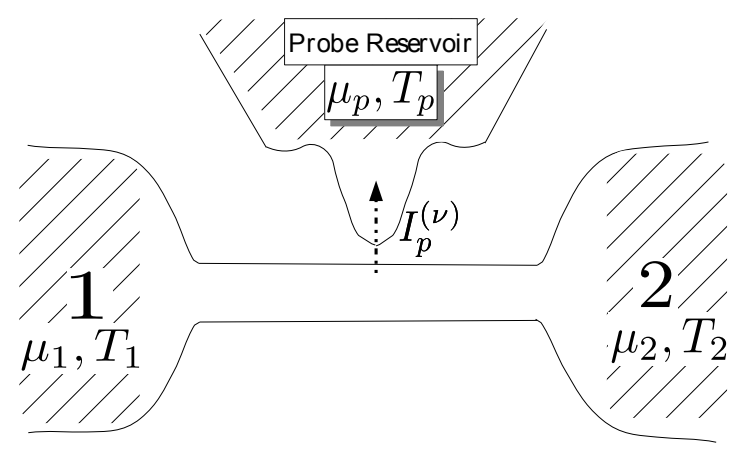

Figure 2.1: Illustration of the measurement setup: The quantum conductor represented above is in a nonequilibrium steady state. A weakly-coupled scanning tunneling probe noninvasively measures the local voltage $\left(\mu_{p}\right)$ and local temperature $\left(T_{p}\right)$ simultaneously: By requiring both a vanishing net charge exchange $\left(I_{p}^{(0)}=0\right)$ and a vanishing net heat exchange $\left(I_{p}^{(1)}=0\right)$ with the system. The nonequilibrium steady state has been prepared, in this particular illustration, via the electrical and thermal bias of the strongly-coupled reservoirs (1 and 2). The measurement method itself is completely general and does not depend upon (a) how such a nonequilibrium steady-state is prepared, (b) how far from equilibrium the quantum electron system is driven, and (c) the nature of interactions within that system.

We define the system's local temperature and voltage using a probe that is weakly coupled via a tunnel barrier. The other end of this scanning probe [16, 75] is the macroscopic electron reservoir whose temperature and voltage are both adjusted until Eq. (2.13) is satisfied. A weakly-coupled probe is a useful theoretical construction for our analysis and the extension of our results beyond the weakcoupling limit is an open question. We explain the physical basis of weak coupling in 2.3.2 and derive some useful formulae. Before proceeding to the main results, we take a short detour which will help us better understand the measurement condition of Eq. (2.13) by considering an example system. 


\subsubsection{Example: Anthracene Junction}

We introduced the nonequilibrium spectrum $f_{s}$ and the probe-system transmission function $\mathcal{T}_{p s}$ in Eqs. 2.3 and 2.4 respectively. It is beneficial to have an intuitive picture of these quantities. The local measurement condition of Eq. (2.13) lends itself to the easiest physical interpretation when the probe is in the broadband limit. We discuss this limit in detail in the subsection 2.5.2 on ideal probes. Succinctly put, the measurement condition just becomes the following statement. We look for an equilibrium system with the distribution $f_{p}$ which give us the same average energy and average occupancy as that of the nonequilibrium system with the distribution $f_{s}$. The spectrum and distribution function of the system are sampled locally by the probe and are experimentally accessible [76].

We consider an anthracene molecular junction driven out of equilibrium by both temperature and voltage biases as shown in Fig. 2.2. The bias conditions were taken to be $T_{1}=100 \mathrm{~K}$ (reservoir 1 is indicated with blue squares), $T_{2}=300 \mathrm{~K}$ (reservoir 2 is indicated with red squares) and $\mu_{2}-\mu_{1}=0.2 \mathrm{eV}$. In the upper panel of fig. 2.2 , we show the voltage and temperature distributions along the molecule as measured by a scanning probe whose tip is $3.5 \AA$ above the plane of the molecule.

There are two representative points on the molecule which are shown in the lower panel of Fig. 2.2. Point 2 illustrates the measurement condition very clearly. One can see that the distribution function for point 2 has two clear steps corresponding to different the chemical potentials of the two reservoirs. The two steps also have different widths corresponding to the different temperatures of the two reservoirs. The measurement idea is then the following: We want to fit a Fermi-Dirac distribution such that it gives the same occupancy and energy as the nonequilibrium distribution (cf. discussion in Ref. [31]). In other words, we want to find an equilibrium distribution $f_{p}$ which gives the same zeroth and first moments of the system's local spectrum. This corresponds to the conditions of zero particle and heat currents as written by the measurement condition in Eq. (2.13). The broadband condition has important implications for measurements which we discuss in section 2.5 on the 

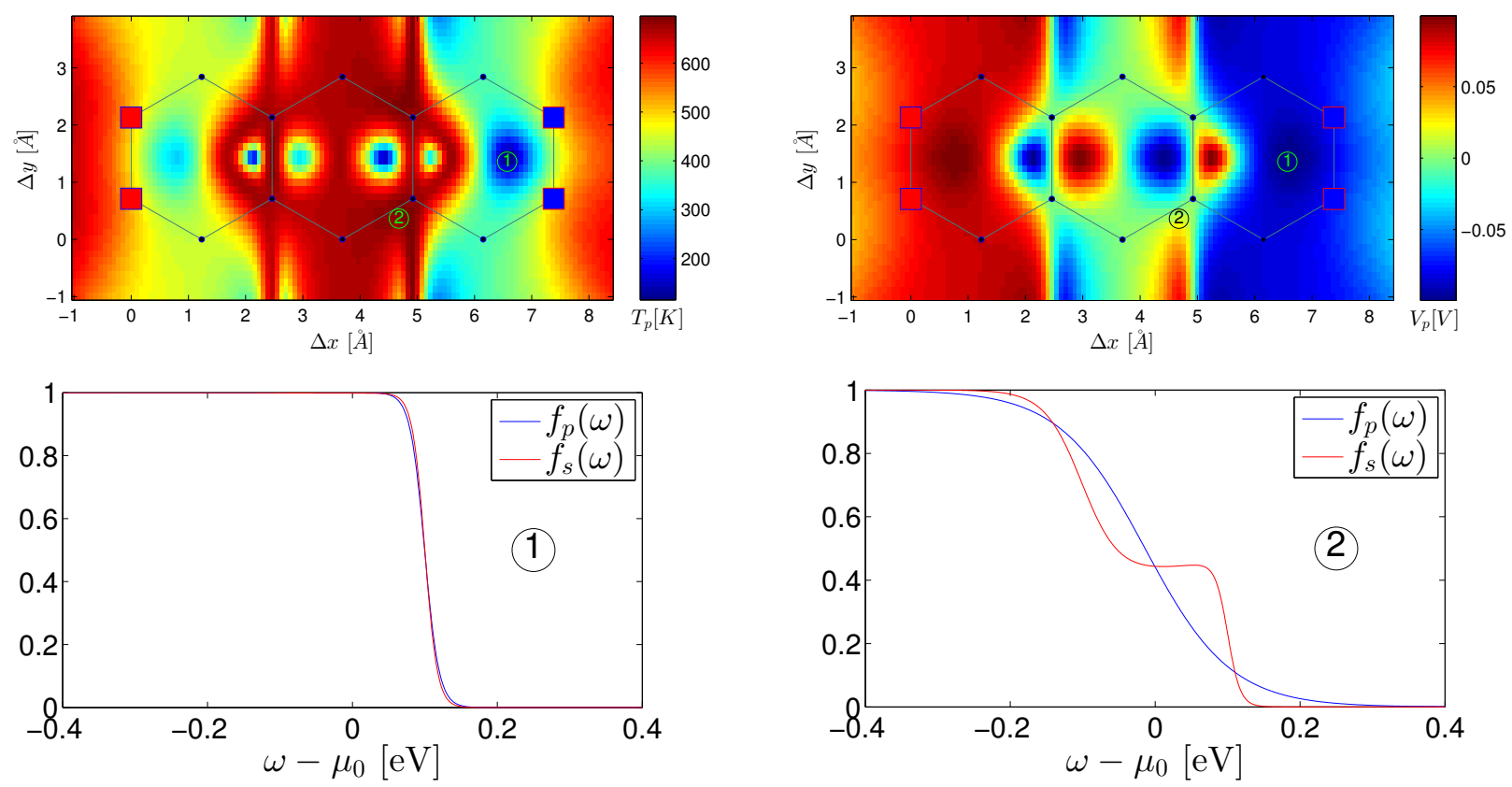

Figure 2.2: Upper panel shows the temperature and voltage variations along the molecular junction as seen by a probe scanning at a height of $3.5 \AA$. Lower panel shows the nonequilibrium distribution of the system $f_{s}$ and the equilibrium Fermi-Dirac distribution $f_{p}$ of the probe for two representative points marked 1 and 2. The measurement finds $f_{p}$ so that the mean energy and occupancy of the probe is the same as that of the nonequilibrium system locally. The distribution $f_{s}$ at point 2 has the clear feature of mixing of two Fermi-Dirac distributions corresponding to the two reservoirs (shown with red and blue squares). 
existence of solutions in the present chapter. We refer the reader also to a related discussion in Ref. [31.

\subsubsection{Noninvasive Measurements}

When the coupling of the probe to the system is weak, we may take $\mathcal{T}_{p s}(\omega)$ in Eq. (2.4) and the local nonequilibrium distribution function $f_{s}(\omega)$ to be independent of the probe temperature $T_{p}$ and chemical potential $\mu_{p}$. While both $\mathcal{T}_{p s}(\omega)$ and $f_{s}(\omega)$ depend upon the local probe-system coupling in an obvious manner, the weak-coupling condition essentially implies that the nonequilibrium steady state of the system is unperturbed by the introduction of the probe terminal. The voltage and temperature of the probe itself play no role in preparing the nonequilibrium steady state. In other words, the probe does not drive the system but merely exchanges energy and particles across a weakly-coupled tunnel barrier and constitutes a noninvasive measurement. A precise analysis of the conditions necessary for a noninvasive probe is given in appendix $B$.

Given a system prepared in a certain nonequilibrium steady state (e.g. by a particular bias of the strongly coupled reservoirs), the currents given by Eq. (2.2) are functions of the probe Fermi-Dirac distribution specified by its temperature and chemical potential

$$
I_{p}^{(\nu)} \equiv I_{p}^{(\nu)}\left(\mu_{p}, T_{p}\right)
$$

It can be seen that the currents are continuous functions of $\mu_{p} \in(-\infty, \infty)$ and $T_{p} \in(0, \infty)$ with continuous gradient vector fields defined by

$$
\nabla I_{p}^{(\nu)} \equiv\left(\frac{\partial I_{p}^{(\nu)}}{\partial \mu_{p}}, \frac{\partial I_{p}^{(\nu)}}{\partial T_{p}}\right) .
$$

With $k_{B}$ set to unity, we compute the gradients of the currents using Eq. 2.2. . We find the gradient of the number current to be

$$
\nabla I_{p}^{(0)}=\left(-\mathcal{L}_{p s}^{(0)},-\frac{\mathcal{L}_{p s}^{(1)}}{T_{p}}\right)
$$


The gradient of the heat current reduces to

$$
\nabla I_{p}^{(1)}=\left(-\mathcal{L}_{p s}^{(1)}-I_{p}^{(0)},-\frac{\mathcal{L}_{p s}^{(2)}}{T_{p}}\right)
$$

where we define the response coefficients $\mathcal{L}_{p s}^{(\nu)}$ as

$$
\begin{aligned}
\mathcal{L}_{p s}^{(\nu)} & \equiv \mathcal{L}_{p s}^{(\nu)}\left(\mu_{p}, T_{p}\right) \\
& =\frac{1}{h} \int_{-\infty}^{\infty} d \omega\left(\omega-\mu_{p}\right)^{\nu} \mathcal{T}_{p s}(\omega)\left(-\frac{\partial f_{p}}{\partial \omega}\right),
\end{aligned}
$$

which are easily seen to be finite' Expressions 2.16) and (2.17) for the current gradients are valid for a noninvasive probe measurement for steady-state transport arbitrarily far from equilibrium and with arbitrary interactions within the quantum system.

Although the coefficients $\mathcal{L}_{p s}^{(\nu)}$ formally resemble the Onsager linear response coefficients [32] of an elastic quantum conductor [77], it is very important to note that we do not make the assumptions of linear response, time-reversal symmetry, local equilibrium, or elastic transport in the above definition of $\mathcal{L}_{p s}^{(\nu)}$ : The system itself may be arbitrarily far from equilibrium with arbitrary inelastic scattering processes. The coefficients above appear naturally when we calculate the gradient fields defined by Eq. 2.15) and the gradient operator is of course given by the first derivatives. Our main results follow from an analysis of the properties of these gradient fields.

\subsection{Uniqueness and the Second Law}

We now turn to one of the central problems which we set out to address: $I_{p}^{(\nu)}\left(\mu_{p}, T_{p}\right)=0$, with $\nu=\{0,1\}$, is a system of coupled nonlinear equations in two variables that defines our local voltage and temperature measurement. There is no a priori reason to expect a unique solution even if a solution exists. We begin

\footnotetext{
${ }^{\dagger} \mathcal{L}_{p s}^{(\nu)}\left(\mu_{p}, T_{p}\right)$ are finite even if $\mathcal{T}_{p s}(\omega)$ and $\omega \mathcal{T}_{p s}(\omega)$ do not obey the finite measure conditions of postulate 1 due to the exponentially decaying tails of the Fermi-derivative. We merely need $\mathcal{T}_{p s}(\omega)$ to grow slower than exponentially for $\omega \rightarrow \pm \infty$.
} 
the section with statements of the second law of thermodynamics and conclude by showing that the uniqueness of the measurement emerges as a consequence.

\subsubsection{Statements of the Second Law}

We note that $\forall \mu_{p} \in(-\infty, \infty)$ and $T_{p} \in(0, \infty)$,

$$
\begin{aligned}
& \mathcal{L}_{p s}^{(0)}\left(\mu_{p}, T_{p}\right)>0 \\
& \mathcal{L}_{p s}^{(2)}\left(\mu_{p}, T_{p}\right)>0,
\end{aligned}
$$

since $\mathcal{T}_{p s}(\omega) \geq 0$, and the measure of $\mathcal{T}_{p s}(\omega)$ and the Fermi-function derivative are both nonzero and strictly positive. This leads to two statements of the second law of thermodynamics, related to the Clausius statement, which are presented in the following two lemmas. The idea is to choose the correct contour for each case and evaluate the line integral over the current gradients given by Eqs. 2.16) and (2.17). A cursory glance at the number current gradient in Eq. (2.16) suggests that the contour should be defined over a constant temperature while the heat current gradient in Eq. (2.17) suggests a line integral over a constant voltage contour.

Lemma 1 The number current contour defined by $I_{p}^{(0)}\left(\mu_{p}, T_{p}\right)=0$ exists for all $T_{p} \in(0, \infty)$ and defines a function $M:(0, \infty) \rightarrow \mathbb{R}$, where $\mu_{p}=M\left(T_{p}\right)$, such that the second law of thermodynamics is obeyed:

$$
\begin{aligned}
& I_{p}^{(0)}\left(\mu_{p}^{\prime}, T_{p}\right)>0, \text { if } \mu_{p}^{\prime}<\mu_{p} \text { and } \\
& I_{p}^{(0)}\left(\mu_{p}^{\prime}, T_{p}\right)<0, \text { if } \mu_{p}^{\prime}>\mu_{p} .
\end{aligned}
$$

Proof. We first show that $I^{(0)}\left(\mu_{p}, T_{p}\right)=0$ is satisfied for all $T_{p} \in(0, \infty)$. For any $T_{p} \in(0, \infty)$, we have

$$
\begin{aligned}
\lim _{\mu_{p} \rightarrow-\infty} I^{(0)}\left(\mu_{p}, T_{p}\right) & =\frac{1}{h} \int_{-\infty}^{\infty} d \omega \mathcal{T}_{p s}(\omega)\left[f_{s}(\omega)\right. \\
& \left.-\lim _{\mu_{p} \rightarrow-\infty} f_{p}(\omega)\right] \\
& =\frac{1}{h} \int_{-\infty}^{\infty} d \omega \mathcal{T}_{p s}(\omega) f_{s}(\omega) \\
& >0
\end{aligned}
$$




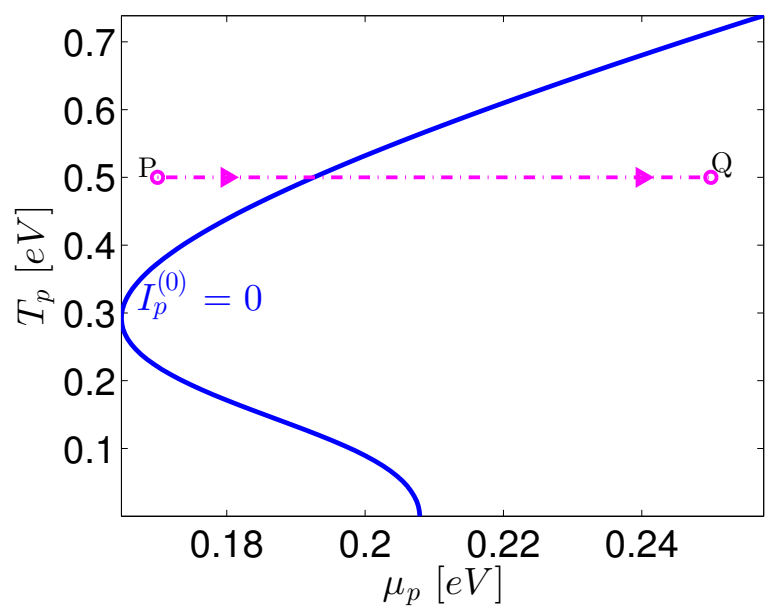

Figure 2.3: Illustration of Lemma 1; The contour $P Q$ shown in magenta cuts the number current contour $I_{p}^{(0)}=0$ (or any $I_{p}^{(0)}=$ constant) exactly once. The contour line from $P$ to $Q$ is at a constant temperature $\left(T_{p}=\right.$ constant $)$ and illustrates the Clausius statement: The number current is monotonically decreasing along $P Q$. The system and bias conditions are detailed in Sec. 2.5.4.

and

$$
\begin{aligned}
\lim _{\mu_{p} \rightarrow \infty} I^{(0)}\left(\mu_{p}, T_{p}\right) & =\frac{1}{h} \int_{-\infty}^{\infty} d \omega \mathcal{T}_{p s}(\omega)\left[f_{s}(\omega)\right. \\
& \left.-\lim _{\mu_{p} \rightarrow \infty} f_{p}(\omega)\right] \\
& =\frac{1}{h} \int_{-\infty}^{\infty} d \omega \mathcal{T}_{p s}(\omega)\left(f_{s}(\omega)-1\right) \\
& <0 .
\end{aligned}
$$

This ensures at least one solution due to the continuity of the currents but does not ensure uniqueness.

We note that $I_{p}^{(0)}$ is monotonically decreasing along $\mathbf{d l}=\left(d \mu_{p}, 0\right)$

$$
\Delta I_{p}^{(0)}=\int_{\mu_{p}}^{\mu_{p}^{\prime}} \nabla I_{p}^{(0)} \cdot \mathbf{d} \mathbf{l}=\int_{\mu_{p}}^{\mu_{p}^{\prime}}-\mathcal{L}_{p s}^{(0)} d \mu_{p}
$$

due to the fact that $\mathcal{L}_{p s}^{(0)}$ is positive, and more explicity:

$$
\begin{aligned}
\Delta I_{p}^{(0)} & =\frac{1}{h} \int_{-\infty}^{\infty} d \omega \mathcal{T}_{p s}(\omega)\left[f_{p}\left(\mu_{p}, T_{p} ; \omega\right)-f_{p}\left(\mu_{p}^{\prime}, T_{p} ; \omega\right)\right] \\
& >0, \text { if } \mu_{p}^{\prime}<\mu_{p} \\
& <0, \text { if } \mu_{p}^{\prime}>\mu_{p} .
\end{aligned}
$$


This implies the existence of a unique solution to $I_{p}^{(0)}\left(\mu_{p}, T_{p}\right)=0$ for every $T_{p} \in$ $(0, \infty)$ which we denote by $\mu_{p}=M\left(T_{p}\right)$, and Eq. 2.20) is implied by Eq. 2.24 .

We also note that the number current $\left[\mu_{p}=M\left(T_{p}\right)\right]$ contour is vertical when the temperature approaches absolute zero, as shown in Fig. 2.3. since $\mathcal{L}_{p s}^{(1)} / T_{p} \rightarrow 0$ as $T_{p} \rightarrow 0$, and implies a vanishing Seebeck coefficient for the probe-system junction near absolute zero.

An "ideal potentiometer" was initially proposed [56] by merely requiring $I_{p}^{(0)}=0$. Subsequently, Büttiker [78, 79] clarified that this definition holds only near absolute zero due to the absence of thermoelectric corrections. Such a voltage probe determines the voltage uniquely at zero temperature in the linear response regime, and is relevant for experiments in mesoscopic circuits [80 83] which are carried out at cryogenic temperatures. However, at higher temperatures and/or larger bias voltages, where the sample may be heated by both the Joule and Peltier effects, thermoelectric corrections to voltage measurements must be considered. Indeed, Bergfield and Stafford [74] argue that an ideal voltage probe must be required to equilibrate thermally with the system $\left(I_{p}^{(1)}=0\right)$ without which "a voltage will develop across the system-probe junction due to the Seebeck effect."

Voltage probes have been used extensively in the theoretical literature to mimic the effects of various scattering processes, such as inelastic scattering [78, 84] 88] and dephasing [89 91] in mesoscopic systems. A modern variation of Büttiker's voltage probe, additionally requiring that the probe exchange no heat current, has been used to model inelastic scattering in quantum transport problems at finite temperature [58, 61, 92 94]. The probe technique, as a model for scattering, has also been extensively studied beyond the linear response regime [95 97].

Lemma 1 implies that a "voltage probe" (defined only by $I_{p}^{(0)}=0$ ) requires the simultaneous specification of a probe temperature $T_{p}$ so that $\mu_{p}=M\left(T_{p}\right)$ is uniquely determined. Fig. 2.3 illustrates that the measured voltage shows a large dependence on the probe temperature. Therefore, it is important to define a simultaneous temperature measurement by imposing $I_{p}^{(1)}\left(\mu_{p}, T_{p}\right)=0$. 
Lemma 2 The heat current contour defined by $I_{p}^{(1)}\left(\mu_{p}, T_{p}\right)=c$, where $\mathrm{c}$ is some constant, obeys the second law of thermodynamics:

$$
\begin{aligned}
I_{p}^{(1)}\left(\mu_{p}, T_{p}^{\prime}\right) & >c, \text { if } T_{p}^{\prime}<T_{p} \\
& <c, \text { if } T_{p}^{\prime}>T_{p}
\end{aligned}
$$

Proof. We follow an analogous argument to lemma 1 and show the monotonicity of $I_{p}^{(1)}\left(\mu_{p}, T_{p}\right)$ along a certain contour in the $\mu_{p}-T_{p}$ plane. Naturally, the contour we choose is along a fixed $\mu_{p}$ [cf. Eq. 2.17] ] since we know that $\mathcal{L}_{p s}^{(2)}$ is positive. Therefore we have $\Delta I_{p}^{(1)}=I_{p}^{(1)}\left(\mu_{p}, T_{p}^{\prime}\right)-I_{p}^{(1)}\left(\mu_{p}, T_{p}\right)=\int_{T_{p}}^{T_{p}^{\prime}} \nabla I_{p}^{(1)}$.dl, where $\mathbf{d l} \mathbf{l}=$ $\left(0, d T_{p}\right)$ and explicitly,

$$
\begin{aligned}
\Delta I_{p}^{(1)} & =\frac{1}{h} \int_{-\infty}^{\infty} d \omega\left(\omega-\mu_{p}\right) \mathcal{T}_{p s}(\omega)\left[f_{p}\left(\mu_{p}, T_{p} ; \omega\right)\right. \\
& \left.\quad-f_{p}\left(\mu_{p}, T_{p}^{\prime} ; \omega\right)\right] \\
& >0, \text { if } T_{p}^{\prime}<T_{p} \\
& <0, \text { if } T_{p}^{\prime}>T_{p} .
\end{aligned}
$$

This implies Eq. (2.25).

We stated lemma 2 with a constant $c$, not necessarily $c=0$, unlike $\oint^{\ddagger}$ lemma 1 . This is because we do not a priori know whether the contour $I_{p}^{(1)}=0$ exists and we derive a necessary and sufficient condition for its existence in Sec 2.5.

Analogous to Lemma 1, Lemma 2 implies that a "temperature probe" [56] (defined only by $I_{p}^{(1)}=0$ ) requires the simultaneous specification of a probe voltage $\mu_{p}$ so that the temperature $T_{p}=\tau_{0}\left(\mu_{p}\right)$ is uniquely determined. Fig. 2.4 illustrates that the measured temperature shows a large dependence on the probe voltage.

\footnotetext{
${ }^{\ddagger}$ Furthermore, the tangent vector [cf. Eq. 2.34] ] along $I_{p}^{(1)}=c$ cannot be of magnitude zero since $\mathcal{L}_{p s}^{(2)}$ is strictly positive for $T_{p} \in(0, \infty)$. Therefore, the contour $I_{p}^{(1)}=c$ doesn't terminate for finite values of $T_{p}$ and $\mu_{p}$. This implies the existence of a function $\tau_{c}:(-\infty, \infty) \rightarrow(0, \infty)$ which defines

$$
T_{p}=\tau_{c}\left(\mu_{p}\right)
$$

for each point on $I_{p}^{(1)}\left(\mu_{p}, T_{p}\right)=c$.
} 


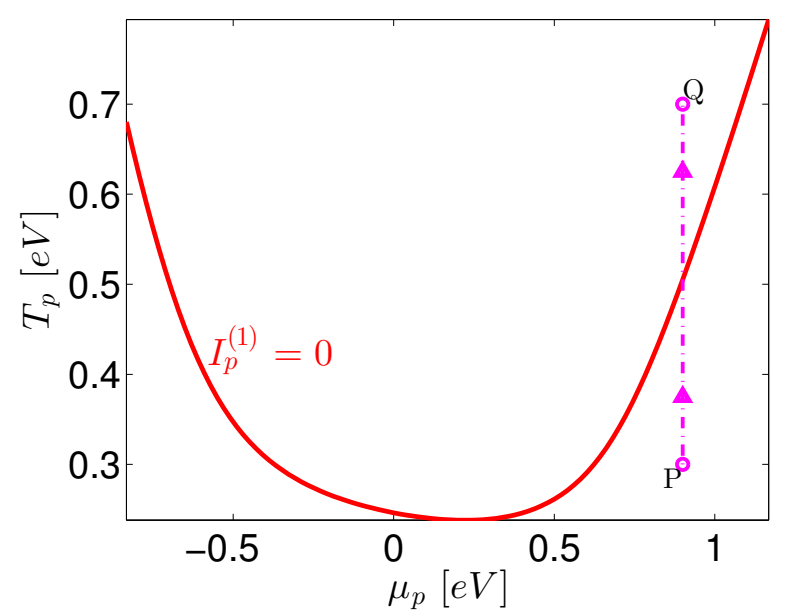

Figure 2.4: Illustration of Lemma 2. The contour $P Q$ shown in magenta cuts $I_{p}^{(1)}=0$ (or any $I_{p}^{(1)}=$ constant) exactly once. Contour $P Q$ is defined by $\mu_{p}=$ constant and illustrates the Clausius statement: The heat current is monotonically decresing along $P Q$. The system and bias conditions are detailed in Sec. 2.5.4.

Therefore, it becomes important to simultaneously measure the voltage by impos$\operatorname{ing} I_{p}^{(0)}=0$. If the temperature probe is not allowed to equilibrate electrically with the system, a temperature difference will build up across the probe-system junction due to the Peltier effect thus leading to an error in the temperature measurement.

Clearly, depending upon the probe voltage, the "temperature probe" could measure any of a range of values thereby rendering the measurement somewhat meaningless (see Fig. 2.4). Analogously, the "voltage probe" could measure any of a range of values depending upon the probe temperature (see Fig. 2.3). Thermoelectric probes (also referred to as dual probes or voltage-temperature probes) treat temperature and voltage measurements on an equal footing and implicitly account for the thermoelectric corrections exactly. Only such a dual probe is in both thermal and electrical equilibrium with the system being measured, and therefore yields an unbiased measurement of both quantities. A mathematical proof of the uniqueness of a voltage and temperature measurement is therefore of fundamental importance.

We may also deduce that $T_{p}=0$ cannot be obtained as a measurement outcome 
since

$$
\begin{aligned}
& \lim _{T_{p} \rightarrow 0} I_{p}^{(1)}\left(\mu_{p}, T_{p}\right)= \int_{-\infty}^{\infty} d \omega\left(\omega-\mu_{p}\right) \mathcal{T}_{p s}(\omega)\left[f_{s}(\omega)\right. \\
&\left.-\lim _{T_{p} \rightarrow 0} f_{p}\left(\mu_{p}, T_{p}\right)\right] \\
&=\int_{-\infty}^{\mu_{p}} d \omega\left(\omega-\mu_{p}\right) \mathcal{T}_{p s}(\omega)\left(f_{s}(\omega)-1\right) \\
&+\int_{\mu_{p}}^{\infty} d \omega\left(\omega-\mu_{p}\right) \mathcal{T}_{p s}(\omega) f_{s}(\omega) \\
&> 0,
\end{aligned}
$$

consistent with the third law of thermodynamics. However, temperatures arbitarily close to absolute zero are, in principle, possible [48].

Lemmas 1 and 2 may be interpreted in terms of the Clausius statement of the second law [3]: "No process is possible whose sole effect is to transfer heat from a colder body to a warmer body." Lemma 2 gives us the direction in which heat will flow [cf. Eq. (2.26)] when the probe is biased away from the point of thermal equilibrium with the system, $I_{p}^{(1)}\left(\mu_{p}, T_{p}\right)=0$ : whenever the probe is hotter than the temperature corresponding to thermal equilibrium, with the chemical potential held constant, heat flows out of the probe and vice versa. Similarly, Lemma 1 gives us the direction in which particle flow occurs when the probe is biased away from the point of electrical equilibrium, $I_{p}^{(0)}\left(\mu_{p}, T_{p}\right)=0$ : whenever the probe is at a higher chemical potential than the one corresponding to electrical equilibrium, with temperature held constant, particles flow out of the probe and vice versa. Here we refer to electrical $(\nu=0$, Lemma 1) and thermal ( $\nu=1$ lemma 2) equilibration of the probe with the system under the local exchange of particles and energy. The system itself may be arbitrarily far from equilibrium and may possess no local equilibrium.

The problem of a unique measurement of a "voltage probe" (defined only by $I_{p}^{(0)}=0$ ) or a "temperature probe" (defined only by $I_{p}^{(1)}=0$ ) has been attempted previously by Jacquet and Pillet [46] for transport beyond linear response and, to our knowledge, is the only work in this direction. However, in Ref. [46], the bias conditions considered are quite restrictive and the result assumes noninteracting electrons. Lemmas 1 and 2 , respectively, generalize the result to arbitrary bias 
conditions, and arbitary interactions within a quantum electron system, while also providing a useful insight via the Clausius statement of the second law of thermodynamics. However, the question we would like to answer in this article pertains to the uniqueness of a thermoelectric probe measurement defined by both $I_{p}^{(0)}=0$ and $I_{p}^{(1)}=0$. A result for such dual probes has been obtained only in the linear response regime and for noninteracting electrons [58].

Theorem 1 The coefficients $\mathcal{L}_{p s}^{(\nu)}$ satisfy the inequality

$$
\mathcal{L}_{p s}^{(0)} \mathcal{L}_{p s}^{(2)}-\left(\mathcal{L}_{p s}^{(1)}\right)^{2}>0
$$

Proof. We may define functions $g(\omega)$ and $h(\omega)$ as

$$
g(\omega)=\sqrt{\mathcal{T}_{p s}(\omega)\left(-\frac{\partial f_{p}}{\partial \omega}\right)}
$$

and

$$
h(\omega)=\left(\omega-\mu_{p}\right) \sqrt{\mathcal{T}_{p s}(\omega)\left(-\frac{\partial f_{p}}{\partial \omega}\right)} .
$$

We note that $g(\omega)$ and $h(\omega)$ belong to $\mathbf{L}^{2}(\mathbb{R})$ (cf. also footnote ${ }^{\dagger}$ on page 35 ). Noting that $g$ and $h$ are real, we apply the Cauchy-Schwarz inequality

$$
\left|\int_{-\infty}^{\infty} d \omega g(\omega) h(\omega)\right|^{2} \leq \int_{-\infty}^{\infty} d \omega|g(\omega)|^{2} \int_{-\infty}^{\infty} d \omega|h(\omega)|^{2} .
$$

The integral appearing on the lhs is $\mathcal{L}_{p s}^{(1)}$ while on the $r h s$ we have the product of $\mathcal{L}_{p s}^{(0)}$ and $\mathcal{L}_{p s}^{(2)}$ respectively. We drop the absolute value on the lhs by noting that $\mathcal{L}_{p s}^{(1)}$ is real and write

$$
\left(\mathcal{L}_{p s}^{(1)}\right)^{2} \leq \mathcal{L}_{p s}^{(0)} \mathcal{L}_{p s}^{(2)}
$$

We drop the equality case above by noting that $g$ and $h$ are linearly independent except for the trivial case when $\mathcal{T}_{p s}(\omega)=0 \forall \omega$ or when the probe coupling is narrowband $\left[\mathcal{T}_{p s}(\omega)=\bar{\gamma} \delta\left(\omega-\omega_{0}\right)\right]$. The latter scenario is discussed again in sec 2.5.3.

The proof of theorem 1 can be easily extended to show the positive-definiteness of the linear response matrices [32] widely used for elastic transport calculations 
(e.g., in Refs. [77, 98). Theorem 1 implies a positive thermal conductance (see e.g., Ref. [98]), which is necessary for positive entropy production consistent with the second law of thermodynamics.

\subsubsection{Uniqueness}

Theorem 2 The local temperature and voltage of a nonequilibrium quantum system, measured by a thermoelectric probe, is unique when it exists.

Proof. The tangent vectors $\mathbf{t}^{(\nu)}$ for $I_{p}^{(\nu)}$ are along

$$
\mathbf{t}^{(0)}=\left(-\frac{\mathcal{L}_{p s}^{(1)}}{T_{p}}, \mathcal{L}_{p s}^{(0)}\right)
$$

and

$$
\begin{aligned}
\mathbf{t}^{(1)} & =\left(\frac{\mathcal{L}_{p s}^{(2)}}{T_{p}},-\mathcal{L}_{p s}^{(1)}-I_{p}^{(0)}\right) \\
& =\left(\frac{\mathcal{L}_{p s}^{(2)}}{T_{p}},-\mathcal{L}_{p s}^{(1)}\right), \text { if } I_{p}^{(0)}=0,
\end{aligned}
$$

respectively, such that we have

$$
\int_{s_{1}}^{s_{2}} d s \frac{\mathbf{t}^{(\nu)} \cdot \nabla I_{p}^{(\nu)}}{\left|\mathbf{t}^{(\nu)}\right|}=0
$$

where $s$ is a scalar parameter that labels points along the contour $I_{p}^{(\nu)}=$ constant.

We now compute the change in $I_{p}^{(1)}$ along the contour $I_{p}^{(0)}=0$. The points along $I_{p}^{(0)}=0$ are labeled by the continuous parameter $\xi$ such that $\mu_{p}=\mu_{p}(\xi)$ and $T_{p}=T_{p}(\xi) . \xi$ is chosen to be increasing with increasing temperature. The change $\Delta I_{p}^{(1)}$ becomes

$$
\begin{aligned}
\Delta I_{p}^{(1)} & =\int_{\xi_{1}}^{\xi_{2}} d \xi \frac{\mathbf{t}^{(0)} \cdot \nabla I_{p}^{(1)}}{\left|\mathbf{t}^{(0)}\right|} \\
& =\int_{\xi_{1}}^{\xi_{2}} d \xi \frac{1}{\left|\mathbf{t}^{(0)}\right| T_{p}}\left(\left(\mathcal{L}_{p s}^{(1)}\right)^{2}-\mathcal{L}_{p s}^{(0)} \mathcal{L}_{p s}^{(2)}\right) \\
& >0 \text { if } \xi_{2}<\xi_{1} \\
& <0 \text { if } \xi_{2}>\xi_{1},
\end{aligned}
$$

due to theorem 1. Therefore $I_{p}^{(1)}=0$ (or for that matter $I_{p}^{(1)}=c$, for any $c$ ) is satisfied atmost at a single point along $I_{p}^{(0)}=0$. 
Theorem 1 is a form of the second law of thermodynamics that gives us the direction in which the heat current flows along the contour $I_{p}^{(0)}=0$ [cf. Eq. 2.36)]. The heat current $I_{p}^{(1)}$ decreases monotonically along the contour $I_{p}^{(0)}=0$. Therefore we may find only one point along $I_{p}^{(0)}=0$ that also satisfies $I_{p}^{(1)}=0$, which implies a unique solution to Eq. 2.13) when it exists.

Indeed, Onsager points out in his 1931 paper [32] that for positive entropy production, the linear response matrix will have to be positive-definite (which translates to our condition in Theorem 1). However, that analysis rests upon the assumption of linear response near equilibrium. Our result in Theorem 1 does not require such a condition for the nonequilibrium state of the system but instead emerges out of the analysis of the currents flowing into a weakly-coupled probe. In addition, we obtain a strict mathematical proof of Onsager's phenomenological statement. We point out that theorem 1 holds even when the physically expected postulate 1 fails, making the uniqueness result in theorem 2 very general (cf. footnote ${ }^{\dagger}$ on page 35). Theorem 1 constitutes the first proof of Onsager's inequality (1931, Ref. [32]) which makes no assumptions about the nature of interactions.

\subsection{Existence}

A unique local measurement of temperature and voltage is only part of our main problem. An equally important part is to derive the conditions for the existence of a solution. The main idea behind this analysis is to follow the number current contour $I_{p}^{(0)}=0$ and ask what happens to the heat current $I_{p}^{(1)}$ as we traverse towards higher and higher temperatures $T_{p} \rightarrow \infty$. We noted that near $T_{p}=0$ the heat current into the probe must be positive, consistent with the third law of thermodynamics [cf. Eq. 2.27]]. Since we know that the heat current is monotonically decreasing along the number current contour (Theorem 2), we could guess whether or not a solution occurs depending upon the asymptotic value of the heat current along that contour as $T_{p} \rightarrow \infty$. In this way, we find a necessary and sufficient condition for the existence of a solution while analyzing the problem for positive temperatures (see 

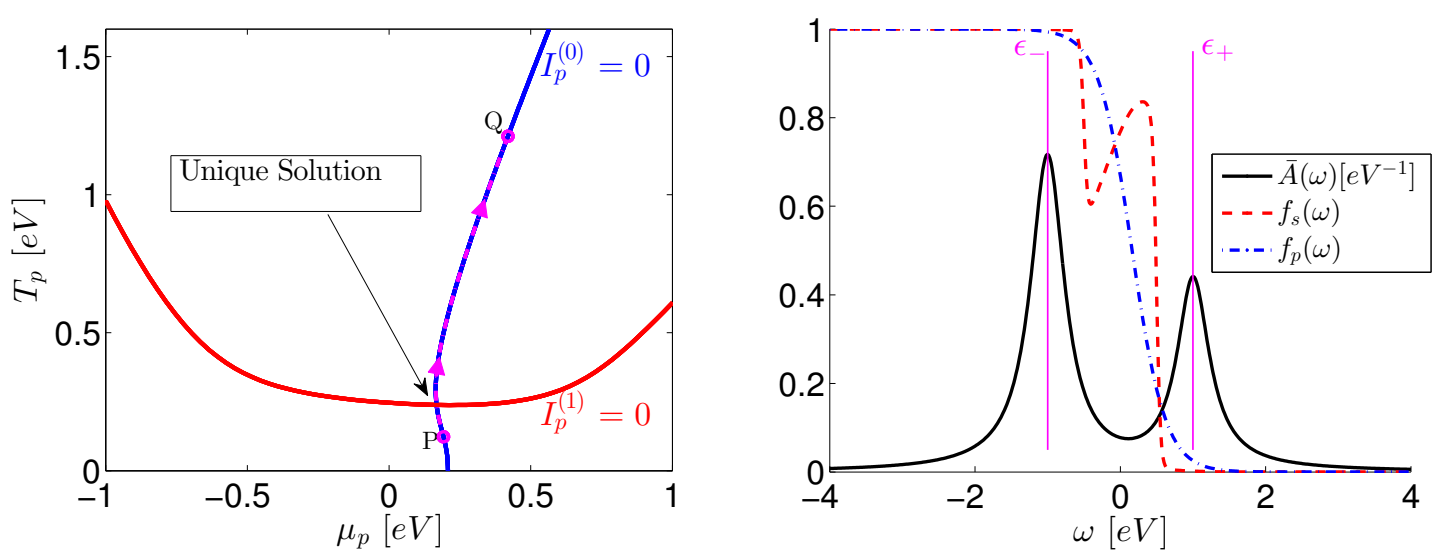

Figure 2.5: Left panel: Illustration of Theorem 2 for positive temperatures. The contour $P Q$ along $I_{p}^{(0)}=0$ (shown in blue) cuts the contour $I_{p}^{(1)}=0$ (shown in red) exactly once. Contour $P Q$ illustrates a certain statement of the second law of thermodynamics: The heat current is monotonically decreasing along $P Q$ (thus implying uniqueness). Right panel: The local spectrum sampled by the probe $\bar{A}(\omega)$ (black), the nonequilibrium distribution function $f_{s}(\omega)$ (red), and the probe Fermi-Dirac distribution $f_{p}(\omega)$ (blue) corresponding to the unique solution in the left panel. The resonances in the spectrum $\bar{A}(\omega)$ correspond to the eigenstates of the closed two-level Hamiltonian (see Sec. 2.5.4) $\epsilon_{ \pm}= \pm 1$ shown in magenta. The Fermi-Dirac distribution is monotonically decreasing with energy, and corresponds to a situation with positive temperature (no net population inversion). The necessary and sufficient condition for the existence of a positive temperature solution is stated in Theorem 3 .

Fig. 2.5 for an illustration of this case). On the other hand, when this condition is not met, one can immediately prove that a negative temperature must satisfy the measurement condition $I_{p}^{(\nu)}=0, \nu=\{0,1\}$. This latter condition corresponds to a system exhibiting local population inversion which leads to negative temperature [99] solutions, as illustrated in Fig. 2.6.

Our results here are again completely general and are valid for electron systems with arbitrary interactions, arbitrary steady-state bias conditions, and for any weakly-coupled probe. However, our analysis here leads us to demarcate between two extremes of the probe-system coupling. We conclude that an ideal probe is one which operates in the broadband limit. A measurement by such a probe depends only on the properties of the system that it couples to and is independent of the 
spectral properties of the probe itself. The broadband limit lends itself to an easier physical interpretation of the population inversion condition as well and we discuss this important limit in Sec. 2.5.2. The other extreme is that of a narrowband probe which is capable of probing the system at just one value of energy, leading to a nonunique measurement (see also the proof of theorem 1), and is discussed in Sec. 2.5.3. Only this pathological case leads to an exception to Theorem 2 .

The simplest system which could, in principle, exhibit population inversion is a two-level system. Therefore, our results, including that of the previous section, have been illustrated by using a two-level system. The details of the nonequilibrium two-level system and its coupling to the thermoelectric probe are given in Sec. 2.5.4.

Our analysis starts with a rearragement of the currents given by Eq. (2.2) and a restatement of the measurement condition [cf. Eq. (2.13)] in terms of energy currents and we also define some useful quantities along the way. We may rewrite the number current in Eq. (2.2) as

$$
I_{p}^{(0)}=\left.\langle\dot{N}\rangle\right|_{f_{s}}-\left.\langle\dot{N}\rangle\right|_{f_{p}}
$$

where

$$
\left.\langle\dot{N}\rangle\right|_{f_{s}} \equiv \frac{1}{h} \int_{-\infty}^{\infty} d \omega \mathcal{T}_{p s}(\omega) f_{s}(\omega)
$$

and similarly

$$
\left.\langle\dot{N}\rangle\right|_{f_{p}} \equiv \frac{1}{h} \int_{-\infty}^{\infty} d \omega \mathcal{T}_{p s}(\omega) f_{p}(\omega) .
$$

The quantitity $\left.\langle\dot{N}\rangle\right|_{f_{s}}$ is the rate of particle flow into the probe from the system while $\left.\langle\dot{N}\rangle\right|_{f_{p}}$ gives the rate of particle flow out of the probe and into the system.

Similarly, the rate of energy flow into the probe from the system is

$$
\left.\langle\dot{E}\rangle\right|_{f_{s}} \equiv \frac{1}{h} \int_{-\infty}^{\infty} d \omega \omega \mathcal{T}_{p s}(\omega) f_{s}(\omega),
$$

while

$$
\left.\langle\dot{E}\rangle\right|_{f_{p}} \equiv \frac{1}{h} \int_{-\infty}^{\infty} d \omega \omega \mathcal{T}_{p s}(\omega) f_{p}(\omega)
$$

gives the rate of energy outflux from the probe back into the system. The net energy current flowing into the probe is given by $I_{p}^{E}=\left.\langle\dot{E}\rangle\right|_{f_{s}}-\left.\langle\dot{E}\rangle\right|_{f_{p}}$. 
The local equilibration conditions in Eq. (2.13) now become

$$
\begin{aligned}
\left.\langle\dot{N}\rangle\right|_{f_{p}} & =\left.\langle\dot{N}\rangle\right|_{f_{s}} \\
\left.\langle\dot{E}\rangle\right|_{f_{p}} & =\left.\langle\dot{E}\rangle\right|_{f_{s}} .
\end{aligned}
$$

The equation for the rate of energy flow above is equivalent to the condition $I_{p}^{(1)}=0$ when $I_{p}^{(0)}=0$ since

$$
\left.I_{p}^{E}\left(\mu_{p}, T_{p}\right) \equiv\langle\dot{E}\rangle\right|_{f_{s}}-\left.\langle\dot{E}\rangle\right|_{f_{p}}=I_{p}^{(1)}+\mu_{p} I_{p}^{(0)} .
$$

The lhs in Eq. (2.42) depends upon the probe parameters (temperature and voltage) while the $r h s$ is fixed for a given nonequilibrium system with a given local distribution function $f_{s}(\omega)$. The probe measures the appropriate voltage and temperature when it exchanges no net charge and energy with the system.

We may introduce a characteristic rate of particle flow [cf. Eq. (2.9)] as

$$
\begin{aligned}
\left.\langle\dot{N}\rangle\right|_{f \equiv 1} & =\frac{1}{h} \int_{-\infty}^{\infty} d \omega \mathcal{T}_{p s}(\omega) \\
& \equiv \frac{\gamma_{p}}{\hbar} .
\end{aligned}
$$

This leads to the following inequalities:

$$
\begin{aligned}
& 0<\left.\langle\dot{N}\rangle\right|_{f_{s}}<\frac{\gamma_{p}}{\hbar}, \\
& 0<\left.\langle\dot{N}\rangle\right|_{f_{p}}<\frac{\gamma_{p}}{\hbar} .
\end{aligned}
$$

The lhs in the inequality for $\left.\langle\dot{N}\rangle\right|_{f_{s}}$ above excludes $f_{s}(\omega) \equiv 0$ while the $r h s$ excludes $f_{s}(\omega)=1 \forall \omega \in \mathbb{R}$, and we retain the strict inequalities imposed by Eq. 2.45) (see also Eqs. 2.11) and (2.12) and the preceding discussion).

We similarly introduce a characteristic rate for the energy flow between the system and probe:

$$
\begin{aligned}
\left.\langle\dot{E}\rangle\right|_{f \equiv 1} & =\frac{1}{h} \int_{-\infty}^{\infty} d \omega \omega \mathcal{T}_{p s}(\omega) \\
& \equiv \frac{\gamma_{p}}{\hbar} \omega_{c},
\end{aligned}
$$

where $\omega_{c}<\infty$ (due to postulate 1) can interpreted as the centroid of the probesample transmission function. We find that $\omega_{c} \rightarrow \infty$ necessarily implies a positive 
temperature solution. We remind the reader that $\omega_{c} \rightarrow-\infty$ is physically impossible due to the principle that any physical system must have a lower bound for the energy $(\langle H\rangle \geq-c$ for some finite $c \in \mathbb{R})$.

The quantities $\left.\langle\dot{N}\rangle\right|_{f_{s}},\left.\langle\dot{N}\rangle\right|_{f_{p}},\left.\langle\dot{N}\rangle\right|_{f \equiv 1},\left.\langle\dot{E}\rangle\right|_{f_{s}},\left.\langle\dot{E}\rangle\right|_{f_{p}},\left.\langle\dot{E}\rangle\right|_{f \equiv 1}$ are all finite due to postulate 1 [cf. Eqs. $2.9+2.12]$ ].

\subsubsection{Asymptotic Properties and Conditions for the Existence of a Solution}

Traversing along $I_{p}^{(0)}=0$ results in a monotonically decreasing heat current $I_{p}^{(1)}$ (Theorem 2). Here, we traverse the contour from low temperatures $\left(T_{p} \rightarrow 0\right)$ to high temperatures $\left(T_{p} \rightarrow \infty\right)$ as discussed in Theorem 2, This implies a monotonically increasing $\left.\langle\dot{E}\rangle\right|_{f_{p}}$ due to Eq. 2.43). We proceed to calculate the asymtotic value of $\left.\langle\dot{E}\rangle\right|_{f_{p}}$ along the number current contour.

Let the asymptotic scaling of $\mu_{p}=M\left(T_{p}\right)$ defined by the contour $I_{p}^{(0)}\left(\mu_{p}, T_{p}\right)=0$ (lemma 1) be

$$
\lim _{T_{p} \rightarrow \infty} \frac{M\left(T_{p}\right)}{T_{p}}=\Lambda
$$

We use the above limiting value to calculate $\left.\langle\dot{N}\rangle\right|_{f_{p}}$ along the contour $\mu_{p}=M\left(T_{p}\right)$ :

$$
\begin{aligned}
\left.\lim _{T_{p} \rightarrow \infty}\langle\dot{N}\rangle\right|_{f_{p}} & =\frac{1}{h} \int_{-\infty}^{\infty} d \omega \mathcal{T}_{p s}(\omega) \\
& \times \lim _{T_{p} \rightarrow \infty} \frac{1}{1+\exp \left\{\left(\frac{\omega-M\left(T_{p}\right)}{T_{p}}\right)\right\}} \\
& =\frac{1}{h} \int_{-\infty}^{\infty} d \omega \mathcal{T}_{p s}(\omega) \frac{1}{1+\exp \{(-\Lambda)\}} \\
& =\frac{1}{1+\exp \{(-\Lambda)\}} \frac{\gamma_{p}}{\hbar} .
\end{aligned}
$$

The above limiting value satisfies the inequality in Eq. 2.45) for any $\Lambda \in \mathbb{R}$. The points on the contour satisfy $\left.\langle\dot{N}\rangle\right|_{f_{p}}=\left.\langle\dot{N}\rangle\right|_{f_{s}}$ by construction, therefore $\Lambda$ is computed from the equation

$$
\frac{1}{1+\exp \{(-\Lambda)\}} \frac{\gamma_{p}}{\hbar}=\left.\langle\dot{N}\rangle\right|_{f_{s}}
$$


It is important to note that the asymptotic scaling defined by Eq. (2.47) does not mean that the scaling is linear. For example, a sublinear scaling $M\left(T_{p}\right)=\alpha T_{p}^{n}$ with $n<1$ merely corresponds to $\Lambda=0$ which could satisfy Eq. (2.49) if the nonequilibrium system is prepared in that way. However, $\Lambda \rightarrow \pm \infty$ do not obey the strict inequality in Eq. (2.45). $\Lambda \rightarrow \infty$ corresponds to a trivial and unphysical nonequilibrium distribution $f_{s}(\omega) \equiv 1$, and likewise, $\Lambda \rightarrow-\infty$ corresponds to $f_{s}(\omega) \equiv 0 \forall \omega$.

The asymtotic value of $\left.\langle\dot{E}\rangle\right|_{f_{p}}$ along the $I_{p}^{(0)}=0$ contour is simply

$$
\begin{aligned}
\left.\lim _{T_{p} \rightarrow \infty}\langle\dot{E}\rangle\right|_{f_{p}} & =\frac{1}{h} \int_{-\infty}^{\infty} d \omega \omega \mathcal{T}_{p s}(\omega) \\
& \times \lim _{T_{p} \rightarrow \infty} \frac{1}{1+\exp \left(\frac{\omega-M\left(T_{p}\right)}{T_{p}}\right)} \\
& =\frac{1}{h} \int_{-\infty}^{\infty} d \omega \omega \mathcal{T}_{p s}(\omega) \frac{1}{1+\exp \{(-\Lambda)\}} \\
& =\frac{1}{1+\exp \{(-\Lambda)\}} \frac{\gamma_{p}}{\hbar} \omega_{c} \\
& =\left.\omega_{c}\langle\dot{N}\rangle\right|_{f_{s} .}
\end{aligned}
$$

Theorem 3 A positive temperature solution exists if and only if there is no net population inversion, i.e., when

$$
\frac{\left.\langle\dot{E}\rangle\right|_{f_{s}}}{\left.\langle\dot{N}\rangle\right|_{f_{s}}}<\omega_{c}
$$

Proof. $\left.\langle\dot{E}\rangle\right|_{f_{p}} /\left.\langle\dot{N}\rangle\right|_{f_{s}}<\left.\langle\dot{E}\rangle\right|_{f_{s}} /\left.\langle\dot{N}\rangle\right|_{f_{s}}$ when $T_{p} \rightarrow 0$ along the contour $I_{p}^{(0)}=0$ [cf. Eq. 2.27) and Eq. 2.43)]. The asymptotic limit of $\left.\langle\dot{E}\rangle\right|_{f_{p}} /\left.\langle\dot{N}\rangle\right|_{f_{s}}$ is $\omega_{c}$ [cf. Eq. [2.50)]. $\left.\langle\dot{E}\rangle\right|_{f_{p}}$ is continuous $\forall \mu_{p} \in(-\infty, \infty), T_{p} \in(0, \infty)$ and is monotonically increasing along $I_{p}^{(0)}=0$ (Theorem 2). We use the intermediate value theorem.

Corollary 3.1 There exists a negative temperature solution for a nonequilibrium system with net population inversion, i.e., when

$$
\frac{\left.\langle\dot{E}\rangle\right|_{f_{s}}}{\left.\langle\dot{N}\rangle\right|_{f_{s}}}>\omega_{c} .
$$



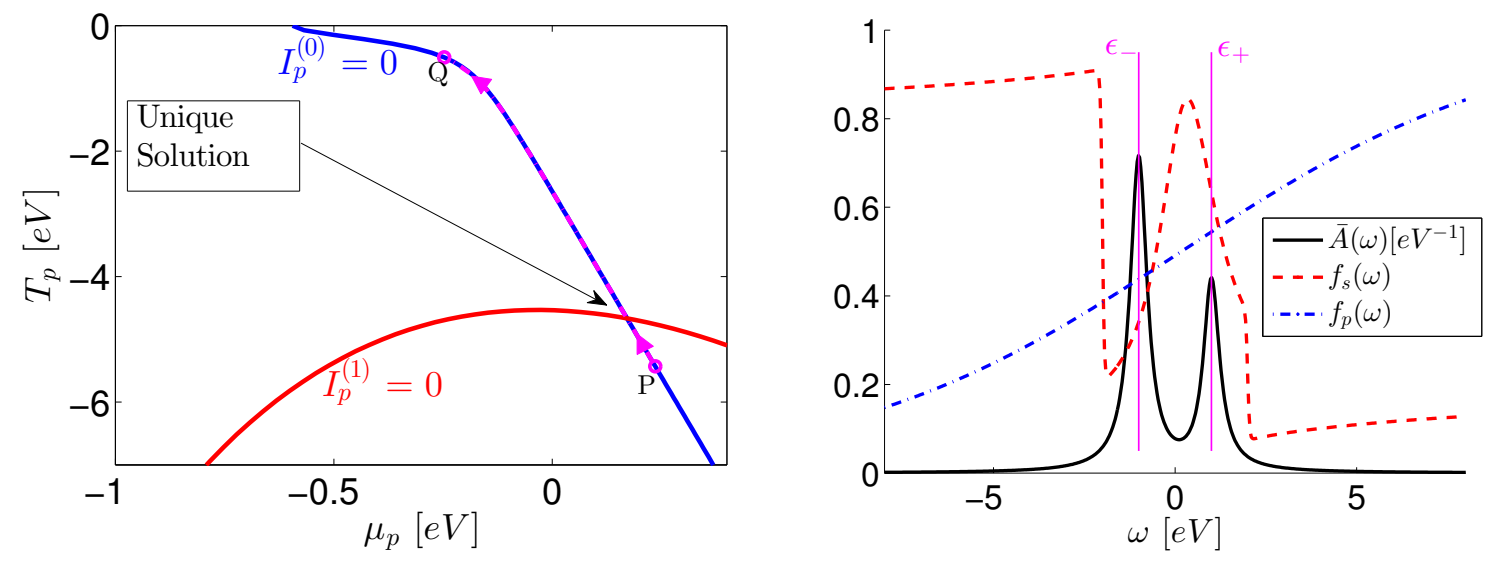

Figure 2.6: Left panel: Illustration of Theorem 2 for negative temperatures. The contour $P Q$ along $I_{p}^{(0)}=0$ (shown in blue) cuts the contour $I_{p}^{(1)}=0$ (shown in red) exactly once. Contour $P Q$ illustrates a certain statement of the second law of thermodynamics: The heat current is monotonically decreasing along $P Q$ (thus implying uniqueness). Right panel: The local spectrum sampled by the probe $\bar{A}(\omega)$ (black, and nearly unchanged from Fig. 2.5), the nonequilibrium distribution function $f_{s}(\omega)$ (red), and the probe Fermi-Dirac distribution $f_{p}(\omega)$ (blue) which corresponds to the unique solution (shown in the left panel). The resonances in the spectrum $\bar{A}(\omega)$ correspond to the eigenstates of the closed two-level Hamiltonian (see Sec. 2.5.4) $\epsilon_{ \pm}= \pm 1$ shown in magenta. The system has a net population inversion, satisfying the conditions of Corollary 3.1, and the probe Fermi-Dirac distribution is monotonically increasing with energy, corresponding to a negative temperature. 
Proof. Let $f_{p}\left(\mu_{p}, T_{p}\right)$ be the Fermi-Dirac distribution with $T_{p}>0$; we define the Fermi-Dirac distribution $f_{p}^{-} \equiv f_{p}\left(\mu_{p},-T_{p}\right)=1-f_{p}$.

$$
\begin{aligned}
I_{p}^{(\nu)}\left(\mu_{p},-T_{p}\right) & =\frac{1}{h} \int_{-\infty}^{\infty} d \omega\left(\omega-\mu_{p}\right)^{\nu} \mathcal{T}_{p s}(\omega)\left[f_{s}(\omega)\right. \\
& =\frac{1}{h} \int_{-\infty}^{\infty} d \omega\left(\omega-\mu_{p}\right)^{\nu} \mathcal{T}_{p s}(\omega)\left[f_{p}(\omega)\right. \\
& \left.-\left(1-f_{s}(\omega)\right)\right] \\
& \frac{1}{h} \int_{-\infty}^{\infty} d \omega\left(\omega-\mu_{p}\right)^{\nu} \mathcal{T}_{p s}(\omega)\left[f_{p}(\omega)-f_{s}^{-}(\omega)\right] \\
& \equiv-I_{p}^{(\nu)-}
\end{aligned}
$$

$I_{p}^{(\nu)-}=0$ with $\nu=\{0,1\}$ is now understood to solve the complementary nonequilibrium system with $f_{s}^{-}(\omega) \equiv 1-f_{s}(\omega)$.

$f_{s}^{-}(\omega)$ is of course a completely valid nonequilibrium distribution function and satisfies Eq. 2.8). We apply Theorem 3 and find that

$$
\begin{aligned}
\left.\langle\dot{E}\rangle\right|_{f_{s}^{-}} & <\left.\omega_{c}\langle\dot{N}\rangle\right|_{f_{s}^{-}} \\
\frac{\gamma_{p}}{\hbar} \omega_{c}-\left.\langle\dot{E}\rangle\right|_{f_{s}} & <\omega_{c}\left(\frac{\gamma_{p}}{\hbar}-\left.\langle\dot{N}\rangle\right|_{f_{s}}\right) \\
-\left.\langle\dot{E}\rangle\right|_{f_{s}} & <-\left.\omega_{c}\langle\dot{N}\rangle\right|_{f_{s}} \\
\left.\langle\dot{E}\rangle\right|_{f_{s}} & >\left.\omega_{c}\langle\dot{N}\rangle\right|_{f_{s}} .
\end{aligned}
$$

For the case that $\left.\langle\dot{E}\rangle\right|_{f_{s}}=\left.\omega_{c}\langle\dot{N}\rangle\right|_{f_{s}}, T_{p}= \pm \infty$, corresponding to $f_{p}=1 / 2$, independent of energy.

\subsubsection{Ideal Probes: The Broadband Limit}

In the broadband limit the probe-system coupling becomes energy independent and we may write $\Gamma^{p}(\omega)=\Gamma^{p}\left(\mu_{0}\right)$. The spectrum of the system, sampled locally by the probe, is given by

$$
\begin{aligned}
\bar{A}(\omega) & \equiv \frac{\operatorname{Tr}\left\{\Gamma^{p}(\omega) A(\omega)\right\}}{\operatorname{Tr}\left\{\Gamma^{p}(\omega)\right\}} \\
& =\frac{\operatorname{Tr}\left\{\Gamma^{p}\left(\mu_{0}\right) A(\omega)\right\}}{\operatorname{Tr}\left\{\Gamma^{p}\left(\mu_{0}\right)\right\}} .
\end{aligned}
$$


The occupancy and energy of the system, respectively, are given by

$$
\begin{aligned}
& \left.\langle N\rangle\right|_{f_{s}}=\int_{-\infty}^{\infty} d \omega \bar{A}(\omega) f_{s}(\omega) \\
& \left.\langle E\rangle\right|_{f_{s}}=\int_{-\infty}^{\infty} d \omega \omega \bar{A}(\omega) f_{s}(\omega) .
\end{aligned}
$$

The measurement conditions in Eq. 2.13 become simply [31]

$$
\begin{aligned}
& \left.\langle N\rangle\right|_{f_{p}}=\left.\langle N\rangle\right|_{f_{s}} \\
& \left.\langle E\rangle\right|_{f_{p}}=\left.\langle E\rangle\right|_{f_{s}} .
\end{aligned}
$$

The above equations imply that an ideal measurement of voltage and temperature constitutes a measurement of the zeroth and first moments of the local energy distribution of the system. That is to say, when the probe is in local equilibrium with the nonequilibrium system, the local occupancy and energy of the system are the same as they would be if the system's local spectrum were populated by the equilibrium Fermi-Dirac distribution $f_{p} \equiv f_{p}\left(\mu_{p}, T_{p}\right)$ of the probe.

We may now write the condition for the existence of a positive temperature solution (Theorem 3) simply as

$$
\frac{\left.\langle E\rangle\right|_{f_{s}}}{\left.\langle N\rangle\right|_{f_{s}}}<\omega_{c}
$$

where $\omega_{c}$ is the centroid of the spectrum given by

$$
\omega_{c}=\int_{-\infty}^{\infty} d \omega \omega \bar{A}(\omega)
$$

The condition in Eq. (2.58) implies the following: Given some nonequilibrium distribution function $f_{s}$, one can have a positive temperature solution if and only if the average energy per particle is smaller than the centroid of the spectrum. In other words, a positive temperature solution exists if and only if there is no net population inversion. Similarly, corollary 3.1 states that there exists a negative temperature solution for a system exhibiting population inversion:

$$
\frac{\left.\langle E\rangle\right|_{f_{s}}}{\left.\langle N\rangle\right|_{f_{s}}}>\omega_{c} .
$$


The advantage of the broadband limit is that one may write the measurement conditions, as well as the condition for the existence of a solution, in terms of the local expectation values of the energy and occupancy directly, instead of using the rate of particle and energy flow into the probe. We also do not need to introduce a "characteristic tunneling rate." We note that $\omega_{c}$ in Eq. 2.59) is the centroid since the local spectrum $\bar{A}$ normalizes to unity within the broadband limit (see appendix A. section A.0.1.

A local measurement by a weakly-coupled broadband thermoelectric probe is ideal in the sense that the result is independent of the properties of the probe, and depends only on the nonequilibrium state of the system and the subsystem thereof sampled by the probe. Such a measurement provides more than just an operational definition of the local temperature and voltage of a nonequilibrium quantum system, since the thermodynamic variables are determined directly by the moments 2.56 of the local (nonequilibrium) energy distribution.

\subsubsection{Nonunique Measurements: The Narrowband Limit}

A narrowband probe is one that samples the system only within a very narrow window of energy. The extreme case of such a probe-system coupling would be a Dirac-delta function:

$$
\Gamma_{p}(\omega)=2 \pi V_{p}^{\dagger} V_{p} \delta\left(\omega-\omega_{0}\right)
$$

which gives $\mathcal{T}_{p s}(\omega)=2 \pi \operatorname{Tr}\left\{V_{p} A(\omega) V_{p}^{\dagger}\right\} \delta\left(\omega-\omega_{0}\right)$ which we write simply as

$$
\mathcal{T}_{p s}(\omega)=\gamma(\omega) \delta\left(\omega-\omega_{0}\right)
$$

where $\gamma(\omega)=2 \pi \operatorname{Tr}\left\{V_{p} A(\omega) V_{p}^{\dagger}\right\}$ has dimensions of energy.

We previously noted that Theorem 1 does not hold for $\mathcal{T}_{p s}$ given by Eq. 2.62. One can verify straightforwardly that, for a probe-sample transmission that is extremely narrow, we will have

$$
\mathcal{L}_{p s}^{(0)} \mathcal{L}_{p s}^{(2)}-\left(\mathcal{L}_{p s}^{(1)}\right)^{2}=0
$$


This results in a nonunique solution since following the proof of theorem 2 would give us [cf. Eq. 2.36) $\Delta I_{p}^{(1)}=0$. In fact, it would lead to a family of solutions.

We may solve for the solution explicitly. The number current reduces to

$$
I_{p}^{(0)}=\frac{\gamma\left(\omega_{0}\right)}{h}\left(f_{p}\left(\omega_{0}\right)-f_{s}\left(\omega_{0}\right)\right)
$$

while the heat current is given by

$$
I_{p}^{(1)}=\left(\omega_{0}-\mu_{p}\right) \frac{\gamma\left(\omega_{0}\right)}{h}\left(f_{p}\left(\omega_{0}\right)-f_{s}\left(\omega_{0}\right)\right)
$$

which trivially vanishes for vanishing number current. Therefore, the family of solutions to the measurement is simply given by

$$
f_{p}\left(\omega_{0} ; \mu_{p}, T_{p}\right)=f_{s}\left(\omega_{0}\right)
$$

which is linear in the $\mu_{p}-T_{p}$ plane and is given by

$$
\mu_{p}=\omega_{0}-T_{p} \log \left(\frac{1-f_{s}\left(\omega_{0}\right)}{f_{s}\left(\omega_{0}\right)}\right)
$$

$f_{s}(\omega)$ has the following explicit form:

$$
f_{s}(\omega)=\frac{\operatorname{Tr}\left\{V_{p} G^{<}(\omega) V_{p}^{\dagger}\right\}}{2 \pi i \operatorname{Tr}\left\{V_{p} A(\omega) V_{p}^{\dagger}\right\}} .
$$

A narrowband probe is therefore unsuitable for thermoelectric measurements. Even if a probe were to sample the system at just two distinct energies $\omega_{1}$ and $\omega_{2}$, theorem 1 would hold and the thermoelectric measurement would be unique. Indeed, the narrowband probe is a pathological case whose only function is to highlight a certain theoretical limitation for the measurement of the temperature and voltage.

\subsubsection{Example: Two-level System}

Net population inversion is essentially a quantum phenomenon, since classical Hamiltonians are generally unbounded above due to the kinetic energy term, i.e., there does not exist a finite $c \in \mathbb{R}$ that satisfies $\langle H\rangle<c$. In other words, $\omega_{c} \rightarrow \infty$ generally holds for classical systems and negative temperatures are not possible. The 


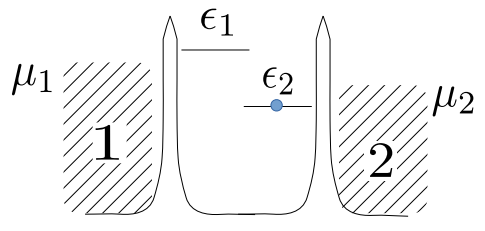

(a)

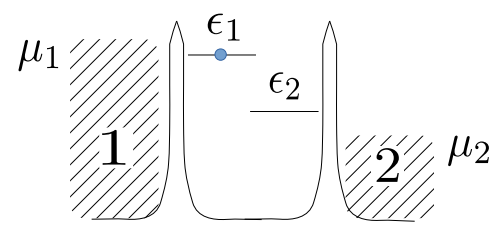

(b)

Figure 2.7: Schematic diagram of a two-level system coupled to two electron reservoirs under a voltage bias. (a) Bias condition not leading to population inversion. (b) Bias condition leading to population inversion due to direct injection into excited state.

simplest quantum system where a net population inversion can be achieved is a twolevel system. We therefore illustrated our results for a two-level system in figs. 2.3 to 2.6 ,

The system Hamiltonian here was taken to be

$$
H=\left[\begin{array}{cc}
\epsilon_{1} & V \\
V^{*} & \epsilon_{2}
\end{array}\right],
$$

whose values were set as $V=\frac{2(1-i)}{3}, \epsilon_{1}=1 / 3$ and $\epsilon_{2}=-1 / 3$, such that the eigenvalues are $\epsilon_{ \pm}= \pm 1$ and units could be read to be in $\mathrm{eV}$. We introduce two reserviors that are strongly coupled locally to each site with $\Gamma_{1}=\operatorname{diag}(0.5,0)$ and $\Gamma_{2}=\operatorname{diag}(0,0.5)$, while the probe coupling is taken as $\Gamma_{p}=\operatorname{diag}(0.01,0.1)$, which is about five times weaker than the coupling to the reservoirs that drive the system.

We used two different bias conditions: (a) To illustrate the case without a net population inversion in figs. 2.3 to 2.5 , the reservoirs had a symmetric $\left(\mu_{1}+\mu_{2}=0\right)$ voltage bias $\mu_{1}-\mu_{2}=1 \mathrm{eV}$; (b) to illustrate the case with a net population inversion in fig. 2.6, the reservoirs had a symmetric voltage bias of $\mu_{1}-\mu_{2}=4 \mathrm{eV}$. The two reservoirs are held at $T=300 \mathrm{~K}$ for both cases.

It has been previously noted that the probe-system coupling strength does not strongly affect the measured temperature and voltage even when varied over several 
orders of magnitude [30], but we remind the reader that our theoretical results depend upon the assumption of a weakly-coupled probe (noninvasive measurements). How weak is weak enough is a different, and perhaps more subtle, theoretical question. We present a more detailed analysis of this point in appendix $B$ on noninvasive measurements. Numerically, however, we do find that the probe measurements are not much altered even when the probe coupling strength is comparable to that of the strongly-coupled reservoirs.

\subsection{Conclusions}

The local temperature and voltage of a nonequilibrium quantum system are defined in terms of the equilibration of a noninvasive thermoelectric probe, locally coupled to the system. The simultaneous temperature and voltage measurement is shown to be unique for any system of fermions in steady state, arbitrarily far from equilibrium, with arbitrary interactions within the system, and the conditions for the existence of a solution are derived. In particular, it is shown that a positive temperature solution exists provided the system does not have a net local population inversion; in the case of population inversion, a unique negative temperature solution is shown to exist. These results provide a firm mathematical foundation for temperature and voltage measurements in quantum systems far from equilibrium.

Our analysis reveals that a simultaneous temperature and voltage measurement is uniquely determined by the local spectrum and nonequilibrium distribution of the

system [cf. Eq. (2.42)], and is independent of the properties of the probe for broadband coupling (ideal probe). Such a measurement therefore provides a fundamental definition of local temperature and voltage which is not merely operational.

In contrast, prior theoretical work relied almost exclusively on operational definitions [46, 56 65] leading to a competing panoply of often contradictory predictions for the measurement of such basic observables as temperature and voltage. Measurements of temperature or voltage, taken separately (see, e.g., Refs. [46, 56]), are shown to be ill-posed: a thermometer out of electrical equilibrium with a system 
produces an error due to the Peltier effect across the probe-sample junction, while a potentiometer out of thermal equilibrium with a system produces an error due to the Seebeck effect. Most importantly, such measurements are not unique even for the same probe and system: the probe's temperature influences the voltage measurement and vice versa.

Our results put the local thermodynamic variables temperature and voltage on a mathematically rigorous footing for fermion systems under very general nonequilibrium steady-state conditions - a necessary first step toward the construction of nonequilibrium thermodynamics [1, 31, 43, 48]. Our analysis includes the effect of interactions with bosonic degrees of freedom (e.g., photons, phonons, etc.) on the fermions. However, the temperatures of the bosons themselves [67, 68] were not addressed in the present analysis. Moreover, we did not explicitly consider magnetic systems, which require separate consideration of the spin degree of freedom and its polarization. Future investigation of probes that exchange bosonic or spin excitations may enable similarly rigorous analyses of local thermodynamic variables in bosonic and magnetic systems, respectively. 


\section{CHAPTER 3}

Coldest Measurable Temperature

This chapter is based on the work published in Ref. [48].

We ask a question motivated by the third law of thermodynamics: What is the coldest possible temperature one can measure in a nonequilibrium quantum system? We have discussed how to measure temperature and voltage in the previous chapter. Most importantly, we realized that temperature and voltage have to be measured simultaneously to ensure uniqueness of the measurement.

Consider the heat current flowing into the probe reservoir $p$ as written in Eq. (2.2)

$$
I_{p}^{(1)}=\frac{1}{h} \int_{-\infty}^{\infty} d \omega\left(\omega-\mu_{p}\right) \mathcal{T}_{p s}(\omega)\left[f_{s}(\omega)-f_{p}(\omega)\right] .
$$

It can be seen that as $T_{p} \rightarrow 0$, the heat current is always positive since $\mathcal{T}_{p s}(\omega) \geq 0$ and $0 \leq f_{s} \leq 1$

$$
\begin{aligned}
\lim _{T_{p} \rightarrow 0} I_{p}^{(1)}= & \frac{1}{h} \int_{-\infty}^{\mu_{p}} d \omega\left(\omega-\mu_{p}\right) \mathcal{T}_{p s}(\omega)\left[f_{s}(\omega)-1\right] \\
& +\frac{1}{h} \int_{\mu_{p}}^{\infty} d \omega\left(\omega-\mu_{p}\right) \mathcal{T}_{p s}(\omega) f_{s}(\omega) \\
& >0
\end{aligned}
$$

since both terms on the rhs are positive and neither can be zero due to our original postulate 1 about the measurability of $\mathcal{T}_{p s}$ and $f_{s}$. This is a statement of the third law of thermodynamics which tells us that it is impossible to cool a system to zero temperature. In fact, it tells us that we will necessarily heat a system at zero temperature. It is also evident from Eq. (3.2) that one cannot obtain a measurement result of $T_{p}=0$ by solving $I_{p}^{(\nu)}=0$ for vanishing particle $(\nu=0)$ and heat currents $(\nu=1)$.

Suppose all the reservoirs are at zero temperature and there is no voltage bias. This of course corresponds to an equilibrium system at zero temperature and there 
is no transport. In this case, the heat current in Eq. (3.2) goes to zero and we obtain the measurement result to be $T_{p}=0$. Even the slightest voltage bias will in fact render the measurement $T_{p}>0$ and thus it is impossible to measure $T_{p}=0$ for nonequilibrium systems. However, we can still ask the question as to what the coldest possible temperature is in a system out of equilibrium. Clearly, applying a voltage bias at zero temperature leads to Joule heating and there can be no thermoelectric cooling to counter it. Therefore we select an idealized situation where one reservoir is at or near absolute zero and another is at finite temperature and there is no voltage bias. Can there then be a local point in the system where a probe can measure zero temperature? We present a detailed analysis of this question in the present chapter.

\subsubsection{Local Temperature and Voltage Measurements}

We briefly review in this section the definitions of a temperature and voltage measurement. This was the primary concern of chapter 2 and the reader may skip this section entirely if they are aware of the results from chapter 2 or may refer to chapter 2 if further clarifications are needed. This section has been added merely to let the chapter stand on its own. The main concern of this chapter is a detailed analysis of the third law scenario. A brief reminder of local temperature and voltage measurements is given below but the reader may also refer to sec. 2.3 and its preceding discussion in 2.2 .

The Green's function formalism provides the following general formula for the particle and heat currents flowing into a reservoir p:

$$
I_{p}^{(\nu)}=-\frac{i}{h} \int_{-\infty}^{\infty} d \omega\left(\omega-\mu_{p}\right)^{\nu} \operatorname{Tr}\left\{\Gamma^{p}(\omega)\left(G^{<}(\omega)+f_{p}(\omega)\left[G^{>}(\omega)-G^{<}(\omega)\right]\right)\right\},
$$

which is valid for arbitrary interactions and for transport arbitrarily far from equilibrium.

A definition for a local electron temperature and voltage measurement on the system that takes into account the thermoelectric corrections was proposed in Ref. [63] by noting that the temperature $T_{p}$ and chemical potential $\mu_{p}$ should be simulta- 
neously defined by the requirement that both the electric current and the electronic heat current into the probe vanish:

$$
I_{p}^{(\nu)}=0, \quad \nu \in\{0,1\}
$$

Eq. (3.3) gives the conditions under which the probe is in local equilibrium with the sample, which is itself arbitrarily far from equilibrium.

Previous analyses [30, 63, 74, 100] have considered this problem within linear response theory, which reduces the system of nonlinear equations (3.3) to a system of equations linear in $T_{p}$ and $\mu_{p}$. In this chapter, we consider a problem that is essentially outside the linear response regime and solve the nonlinear system of equations 3.3 numerically. One reservoir at zero temperature and another at a finite temperature entirely invalidates the expressions obtained within the linear response regime which in turn rely upon the Taylor series expansions of the Fermi function.

It was shown in Ref. [31] that Eq. (3.3) can be written in terms of the local properties of the nonequilibrium system. The mean local spectrum sampled by the probe was defined as

$$
\bar{A}(\omega ; \mathbf{x}) \equiv \frac{\operatorname{Tr}\left\{\Gamma^{p}(\omega ; \mathbf{x}) A(\omega)\right\}}{\operatorname{Tr}\left\{\Gamma^{p}(\omega ; \mathbf{x})\right\}}
$$

where $\mathbf{x}$ is the position of the probe and $A(\omega)=i\left(G^{r}(\omega)-G^{a}(\omega)\right) / 2 \pi$ is the spectral function of the nonequilibrium system. Motivated by the relation at equilibrium, $G_{e q}^{<}(\omega)=2 \pi i A(\omega) f_{e q}(\omega)$, the local nonequilibrium distribution function (sampled by the probe) was defined as

$$
f_{s}(\omega ; \mathbf{x}) \equiv \frac{\operatorname{Tr}\left\{\Gamma^{p}(\omega ; \mathbf{x}) G^{<}(\omega)\right\}}{2 \pi i \operatorname{Tr}\left\{\Gamma^{p}(\omega ; \mathbf{x}) A(\omega)\right\}}
$$

The mean local occupancy of the system orbitals sampled by the probe is [31]

$$
\langle N(\mathbf{x})\rangle=\int_{-\infty}^{\infty} d \omega \bar{A}(\omega) f_{s}(\omega)
$$

and similarly, the mean local energy of the system orbitals sampled by the probe is 31 .

$$
\langle E(\mathbf{x})\rangle=\int_{-\infty}^{\infty} d \omega \omega \bar{A}(\omega) f_{s}(\omega) .
$$


Eqs. 3.4 3.5) allow us to rewrite Eq. (3.3) in a form analogous to the two-terminal Landauer-Büttiker formula

$$
I_{p}^{(\nu)}=\frac{1}{h} \int_{-\infty}^{\infty} d \omega\left(\omega-\mu_{p}\right)^{\nu} 2 \pi \operatorname{Tr}\left\{\Gamma^{p}(\omega) A(\omega)\right\}\left[f_{s}(\omega)-f_{p}(\omega)\right] .
$$

It was noted that, for the case of maximum local coupling, $\left[\Gamma^{p}(\omega)\right]_{i j}=\Gamma^{p}(\omega) \delta_{i n} \delta_{j n}$, where $i, j$ and $n$ label states in the one-particle Hilbert space of the nanostructure, the quantities $\bar{A}(\omega)=A_{n n}(\omega)$ and $f_{s}(\omega)$ become independent of the probe coupling, and can be related by the familiar equilibrium-type formula, $G_{n n}^{<}(\omega)=2 \pi i A_{n n}(\omega) f_{s}(\omega)$, even though the system is out of equilibrium.

In the broad-band limit $\Gamma^{p}(\omega) \approx \Gamma^{p}\left(\mu_{0}\right)$, where $\mu_{0}$ is the equilibrium Fermi energy of the system, we may write $\operatorname{Tr}\left\{\Gamma^{p}\left(\mu_{0}\right)\right\}=\bar{\Gamma}^{p}$ so that $\bar{A}(\omega)=\operatorname{Tr}\left\{\Gamma^{p}\left(\mu_{0}\right) A(\omega)\right\} / \bar{\Gamma}^{p}$. From Eq. (3.8), we have

$$
I_{p}^{(\nu)}=\frac{\bar{\Gamma}^{p}}{\hbar} \int_{-\infty}^{\infty} d \omega\left(\omega-\mu_{p}\right)^{\nu} \bar{A}(\omega)\left[f_{s}(\omega)-f_{p}(\omega)\right] .
$$

It was noted that the equilibrium condition of Eq. (3.3) now implies that the mean local occupancy and energy of the nonequilibrium system are the same as if its nonequilibrium spectrum $\bar{A}(\omega)$ were populated by the equilibrium Fermi-Dirac distribution of the probe $f_{p}(\omega)$ :

$$
\begin{gathered}
\left.\langle N\rangle\right|_{f_{p}}=\left.\langle N\rangle\right|_{f_{s}} \\
\left.\langle E\rangle\right|_{f_{p}}=\left.\langle E\rangle\right|_{f_{s}},
\end{gathered}
$$

i.e., the probe equilibrates with the system in such a way that $f_{p}(\omega)$ satisfies the constraints imposed by Eqs. (3.10) and (3.11). A local temperature (and voltage) measurement therefore reveals information about the local energy and charge distribution of the system. The existence and uniqueness of the distribution $f_{p}(\omega)$ was shown [41] in chapter 2 and important connections to the second law of thermodynamics were established. We also developed the notion of an ideal probe in chapter 2 and noted that such a probe operates in the broadband limit [cf. discussion in 2.5 .2 . 


\subsection{Local Temperatures near Absolute Zero}

Using arguments involving NEGF identities (see e.g., Ref. [101]), one can show that the total current into the probe is the sum of an elastic contribution and an inelastic contribution:

$$
I_{p}^{(\nu)}=\left.I_{p}^{(\nu)}\right|_{\mathrm{el}}+\left.I_{p}^{(\nu)}\right|_{\mathrm{in}}
$$

The elastic contribution to the current is [77, 102]

$$
\left.I_{p}^{(\nu)}\right|_{\mathrm{el}}=\frac{1}{h} \sum_{\alpha} \int_{-\infty}^{\infty} d \omega\left(\omega-\mu_{p}\right)^{\nu} \mathcal{T}_{p \alpha}(\omega)\left[f_{\alpha}(\omega)-f_{p}(\omega)\right]
$$

where the elastic transmission function is given by [9, 98, 103, 104]

$$
\mathcal{T}_{p \alpha}(\omega)=\operatorname{Tr}\left\{\Gamma^{p}(\omega) G^{r}(\omega) \Gamma^{\alpha}(\omega) G^{a}(\omega)\right\}
$$

The inelastic contribution to the current is

$$
\begin{aligned}
\left.I_{p}^{(\nu)}\right|_{\text {in }}= & -\frac{i}{h} \int_{-\infty}^{\infty} d \omega\left(\omega-\mu_{p}\right)^{\nu} \\
& \times \operatorname{Tr}\left\{\Gamma^{p} G^{r}\left[\left(1-f_{p}\right) \Sigma_{\text {in }}^{<}+f_{p} \Sigma_{\text {in }}^{>}\right] G^{a}\right\}
\end{aligned}
$$

where $\Sigma_{\text {in }}$ is the self-energy due to electron-electron and electron-phonon interactions.

Our analyses in this article consider a quantum conductor that is placed in contact with two electron reservoirs: a cold reservoir $R 1$ and a hot reservoir $R 2$. We are interested in the limiting case where reservoir $R 1$ is held near absolute zero $\left(T_{1} \rightarrow 0\right)$ while $R 2$ is held at finite temperature $\left(T_{2}=100 \mathrm{~K}\right.$ in our simulations $)$. We assume no electrical bias $\left(\mu_{1}=\mu_{2}=\mu_{0}\right)$.

Transport in this regime occurs within a narrow thermal window close to the Fermi energy of the reservoirs, and will thus be dominated by elastic processes, so that the contribution to the probe current from Eq. 3.15 can be neglected. Typically,

$$
\left.I_{p}^{(\nu)}\right|_{\text {in }} /\left.I_{p}^{(\nu)}\right|_{\mathrm{el}} \sim \mathcal{O}\left(k_{B} T / \Delta E\right)^{2}
$$

due to the suppression of inelastic electron scattering by the Pauli exclusion principle at low temperatures, where $\Delta E$ is a characteristic electronic energy scale of the 
problem: $\Delta E=\varepsilon_{F}$ for bulk systems, $\Delta E=\max \left\{\Gamma, \varepsilon_{F}-\varepsilon_{\text {res }}\right\}$ in the resonant tunneling regime. It should be noted that, although the transport energy window is small, this problem is essentially outside the scope of linear response theory owing to the large discrepancies in the derivatives of the Fermi functions of the two reservoirs. Therefore, the problem has to be addressed with the full numerical evaluation of the currents given by Eq. (3.13).

A pure thermal bias, such as the one considered in this article, has been shown to lead to temperature oscillations in small molecular junctions [63] and 1-D conductors [59, 105]. Temperature oscillations have been predicted in quantum coherent conductors such as graphene [100], which allow the oscillations to be tuned (e.g., by suitable gating) such that they can be resolved under existing experimental techniques [52 55] of SThM. More generally, quantum coherent temperature oscillations can be obtained for quantum systems driven out of equilibrium due to external fields [60, 62] as well as chemical potential [74] and temperature bias of the reservoirs. In practice, the thermal coupling of the probe to the environment sets limitations on the resolution of a scanning thermoelectric probe [63]. However, in this article, we ignore the coupling of the probe to the ambient environment, in order to highlight the theoretical limitations on temperature measurements near absolute zero.

In evaluating the expressions for the currents in Eq. (3.13) within elastic transport theory, we encounter integrals of the form $\int_{-\infty}^{\infty} d \omega F(\omega)\left(f_{2}(\omega)-f_{1}(\omega)\right)$. We use the Sommerfeld series given by

$$
\begin{aligned}
& \int_{-\infty}^{\infty} d \omega F(\omega)\left(f_{2}(\omega)-f_{1}(\omega)\right)=\int_{\mu_{1}}^{\mu_{2}} d \omega F(\omega) \\
& +2 \sum_{k} \Theta(k+1)\left[\left(k_{B} T_{2}\right)^{k+1} F^{(k)}\left(\mu_{2}\right)-\left(k_{B} T_{1}\right)^{k+1} F^{(k)}\left(\mu_{1}\right)\right], \\
& \quad k \in\{1,3,5, \ldots\},
\end{aligned}
$$

where we use the symbol $\Theta$ that relates to the Riemann-Zeta function $\zeta$ as

$$
\Theta(k+1)=\left(1-\frac{1}{2^{k}}\right) \zeta(k+1)
$$

and $f_{\alpha}(\omega)$ is the Fermi-Dirac distribution of reservoir $\alpha$. The second term on the $r h s$ of Eq. 3.17) accounts for the exponential tails in $\left(f_{2}(\omega)-f_{1}(\omega)\right)$, and its contribution 
depends on the changes to the function $F(\omega)$ in the neighborhoods of $\omega=\mu_{1}$ and $\omega=\mu_{2}$, and can generally be truncated using a Taylor series expansion for most well-behaved functions $F(\omega)$. The lhs of Eq. (3.17) is bounded if $F(\omega)$ grows slower than exponentially for $\omega \rightarrow \pm \infty$ and is satisfied by the current integrals in Eq. (3.13).

\subsubsection{Constant Trasmissions}

In order to make progress analytically, we consider first the case of constant transmissions:

$$
\mathcal{T}_{p \alpha}(\omega)=\mathcal{T}_{p \alpha}\left(\mu_{0}\right) \equiv \mathcal{T}_{p \alpha}
$$

This is a reasonable assumption because the energy window involved in transport is of the order of the thermal energy of the hot reservoir $\left(k_{B} T_{2} \approx 25 \mathrm{meV}\right.$, at room temperature) and we may expect no great changes to the transmission function. In this case the series (3.17) for the number current contains no temperature terms at all, while the heat current contains terms quadratic in the temperature. It is easy to see that the expression for the number current into the probe becomes

$$
I_{p}^{(0)}=\frac{1}{h} \sum_{\alpha} \mathcal{T}_{p \alpha}\left(\mu_{\alpha}-\mu_{p}\right)
$$

and the heat current into the probe is given by

$$
I_{p}^{(1)}=\frac{1}{h} \sum_{\alpha}\left(\mathcal{T}_{p \alpha} \frac{\left(\mu_{\alpha}-\mu_{p}\right)^{2}}{2}+\frac{\pi^{2} k_{B}^{2}}{6} \mathcal{T}_{p \alpha}\left(T_{\alpha}^{2}-T_{p}^{2}\right)\right) .
$$

Eq. 3.20 does not depend on the temperature and can be solved readily:

$$
\mu_{p}=\mu_{0}
$$

since $\mu_{1}=\mu_{2}=\mu_{0}$ and Eq. 3.21 is solved by

$$
T_{p}=\sqrt{\frac{\mathcal{T}_{p 1} T_{1}^{2}+\mathcal{T}_{p 2} T_{2}^{2}}{\mathcal{T}_{p 1}+\mathcal{T}_{p 2}}}
$$


In this article, we are primarily interested in temperature measurements near absolute zero and work in the limit $T_{1} \rightarrow 0$ which yields

$$
T_{p}=\sqrt{\frac{\mathcal{T}_{p 2}}{\mathcal{T}_{p 1}+\mathcal{T}_{p 2}}} T_{2}
$$

We have $T_{p} \rightarrow 0$ as $\mathcal{T}_{p 2} \rightarrow 0$. Indeed, when the system is decoupled from the hot reservoir $R 2$, the probe would read the temperature of reservoir $R 1$.

\subsubsection{Transmission Node}

The analysis of the previous section suggests that a suppression in the transmission from the finite-temperature reservoir $R 2$ results in probe temperatures in the vicinity of absolute zero. In quantum coherent conductors, destructive interference gives rise to nodes in the transmission function. In this section, we consider a case where the transmission from $R 2$ into the probe has a node at the Fermi energy. In the vicinity of such a node, generically, the transmission probability varies quadratically with energy:

$$
\mathcal{T}_{p 2}(\omega)=\frac{1}{2} \mathcal{T}_{p 2}^{(2)}\left(\omega-\mu_{0}\right)^{2}
$$

while the transmission from the cold reservoir $R 1$ may still be treated as a constant:

$$
\mathcal{T}_{p 1}(\omega)=\mathcal{T}_{p 1}
$$

Applying the Sommerfeld series (3.17) for the number current gives us

$$
\begin{aligned}
I_{p}^{(0)}= & \frac{1}{h}\left(\mathcal{T}_{p 1}\left(\mu_{0}-\mu_{p}\right)-\frac{\mathcal{T}_{p 2}^{(2)}}{6}\left(\mu_{p}-\mu_{0}\right)^{3}\right. \\
& \left.+\frac{\pi^{2}}{6} \mathcal{T}_{p 2}^{(2)}\left(\mu_{0}-\mu_{p}\right) k_{B}^{2} T_{p}^{2}\right)
\end{aligned}
$$

where the $k_{B} T_{2}$ term is still missing since the first derivative of $\mathcal{T}_{p 2}(\omega)$ vanishes at $\mu_{2}=\mu_{0}$. We note that Eq. (3.27) admits a single real root at

$$
\mu_{p}=\mu_{0}
$$


With this solution, we can write down the equation for the heat current as

$$
I_{p}^{(1)}=\frac{1}{h}\left(\frac{\pi^{2} k_{B}^{2}}{6} \mathcal{T}_{p 1}\left(T_{1}^{2}-T_{p}^{2}\right)+\frac{7}{8} \frac{\pi^{4} k_{B}^{4}}{15} \mathcal{T}_{p 2}^{(2)}\left(T_{2}^{4}-T_{p}^{4}\right)\right),
$$

which gives us a simple quadratic equation in $T_{p}^{2}$. We note that the above equation is monotonically decreasing in $T_{p}$ for all positive values of temperature. There exists a unique solution to Eq. 3.29 in the interval $T_{1}<T_{p}<T_{2}$, since $I_{p}^{(1)}\left(T_{p}\right)$ undergoes a sign change between these two values, and is also the only positive solution due to monotonicity. Physically, this solution is reasonable since we expect a temperature measurement to be within the interval $\left(T_{1}, T_{2}\right)$ in the absence of an electrical bias. It is straight-forward to write down the exact solution? to Eq. (3.29). However, we simplify the expression for $T_{p}$ by noting that

$$
\mathcal{T}_{p 2}^{(2)}\left(k_{B} T_{2}\right)^{2} \ll \mathcal{T}_{p 1},
$$

that is, the transmission into the probe from $R 2$ within a thermal energy window $k_{B} T_{2}$ in the presence of a node, is small in comparison to the transmission from $R 1$. The approximate solution for $T_{p}$ then becomes

$$
T_{p}=\sqrt{\frac{7 \pi^{2}}{20} \frac{\mathcal{T}_{p 2}^{(2)}\left(k_{B} T_{2}\right)^{2}}{\mathcal{T}_{p 1}}} T_{2},
$$

where, as before, we have taken the limiting case where $T_{1} \rightarrow 0$.

\subsubsection{Higher-order Destructive Interference}

Although a generic node obtained in quantum coherent transport depends quadratically on the energy, it is possible to obtain higher-order "supernodes" in some

* The exact solution to the Eq. $\left[3.29\right.$ with $T_{1} \rightarrow 0$ is

$$
T_{p}=T_{2}\left(\frac{\sqrt{1+4 \lambda_{1}^{2}}-1}{2 \lambda_{1}}\right)^{\frac{1}{2}}
$$

where $\lambda_{1}$ is defined in Eq. 3.38 and simplifies to

$$
\lambda_{1}=\frac{7 \pi^{2}}{20} \frac{\mathcal{T}_{p 2}^{(2)}\left(k_{B} T_{2}\right)^{2}}{\mathcal{T}_{p 1}}
$$

a factor that appears in Eq. 3.31. 
systems 98 . In the vicinity of such a supernode, the transmission function can be written as

$$
\mathcal{T}_{p 2}(\omega)=\left.\frac{1}{2 n !} \mathcal{T}_{p 2}^{(2 n)}\right|_{\omega=\mu_{0}}\left(\omega-\mu_{0}\right)^{2 n}
$$

while the transmission from $R 1$ may still be approximated by Eq. (3.26). Exact expressions for the currents can again be evaluated using Eq. (3.17). The expression for the number current becomes

$$
\begin{aligned}
& I_{p}^{(0)}=\frac{1}{h}\left(\mathcal{T}_{p 1}\left(\mu_{0}-\mu_{p}\right)-\frac{\mathcal{T}_{p 2}^{(2 n)}}{(2 n+1) !}\left(\mu_{p}-\mu_{0}\right)^{2 n+1}\right. \\
& \left.+2 \sum_{k \in \text { odd }} \Theta(k+1)\left[\left(k_{B} T_{2}\right)^{k+1} \mathcal{T}_{p 2}^{(k)}\left(\mu_{0}\right)-\left(k_{B} T_{p}\right)^{k+1} \mathcal{T}_{p 2}^{(k)}\left(\mu_{p}\right)\right]\right),
\end{aligned}
$$

and we have

$$
\mathcal{T}_{p 2}^{(k)}\left(\mu_{0}\right)=0, \quad \forall k \in\{1,3,5, \ldots\}
$$

Now, with

$$
\mu_{p}=\mu_{0},
$$

every term on the rhs of Eq. (3.33) vanishes. Taking $\mu_{p}=\mu_{0}$, we proceed to write the equation for the heat current. Using Eq. (3.17) with $F(\omega)=\left(\omega-\mu_{0}\right) \mathcal{T}_{p \alpha}(\omega)$, we obtain only one nonvanishing derivative for each reservoir, that is, $F^{(1)}\left(\mu_{0}\right)=\mathcal{T}_{p 1}\left(\mu_{0}\right)$ for $R 1$ and $F^{(2 n+1)}\left(\mu_{0}\right)=(2 n+1) \mathcal{T}_{p 2}^{(2 n)}$ for $R 2$. Therefore,

$$
\begin{aligned}
I_{p}^{(1)}= & \frac{2}{h}\left(\Theta(2) \mathcal{T}_{p 1}\left[\left(k_{B} T_{1}\right)^{2}-\left(k_{B} T_{p}\right)^{2}\right]\right. \\
& \left.+(2 n+1) \Theta(2 n+2) \mathcal{T}_{p 2}^{(2 n)}\left[\left(k_{B} T_{2}\right)^{2 n+2}-\left(k_{B} T_{p}\right)^{2 n+2}\right]\right),
\end{aligned}
$$

which is a polynomial equation in $T_{p}$ of degree $(2 \mathrm{n}+2)$. We can rewrite Eq. (3.36) as a polynomial $p(x)$ in $x=T_{p} / T_{2}$ :

$$
p(x)=x^{2}+\lambda_{n} x^{2 n+2}-\lambda_{n}=0,
$$

where we have taken $T_{1} \rightarrow 0$ for $R 1$, and $\lambda_{n}$ is a dimensionless quantity given by

$$
\lambda_{n}=(2 n+1) \frac{\Theta(2 n+2)}{\Theta(2)}\left(\frac{\mathcal{T}_{p 2}^{(2 n)}\left(k_{B} T_{2}\right)^{2 n}}{\mathcal{T}_{p 1}}\right) .
$$

We will have $\lambda_{n} \ll 1$ for a suitable energy window set by $k_{B} T_{2}$, since the transmission into the probe from $R 2$ suffers destructive interference at the Fermi energy $\mu_{0}$. If 
the thermal energy is large enough, then this approximation may no longer hold. In any case, it is possible to define a temperature $T_{2}$ so that this approximation is strongly valid. Under the validity of this approximation, the solution to Eq. (3.37) can be written using perturbation theory as

$$
x=\sqrt{\lambda_{n}}\left(1+\mathcal{O}\left(\lambda_{n}^{n+1}\right)\right),
$$

with corrections that are of much higher-order in $\lambda_{n}$. The solution for $T_{p}$ given in Eq. (3.39) reduces to Eq. (3.24) for the case with constant transmissions by setting $n=0$, and to the approximate result obtained in Eq. 3.31 in the presence of a node by setting $n=1$. We note that higher-order interference effects cause the probe temperature to decay more rapidly with respect to $T_{2}$ since $T_{p} \sim T_{2}^{n+1}$, that is, when $T_{2}$ is halfed, $T_{p}$ is reduced by a factor of $2^{n+1}$. Now, if we consider the limiting case where $R 2$ is also cooled to absolute zero, $T_{2} \rightarrow 0$, Eq. (3.39) implies that $T_{p} \rightarrow 0$ at least as quickly as $T_{2}$ (in the absence of destructive interference, i.e., $n=0$ ) or quicker (when there is destructive interference, i.e., $n \geq 1$ ).

\subsubsection{Uniqueness and the Second Law}

It should be noted that the polynomial given in Eq. (3.37) is monotonically increasing for all positive $x$ and furthermore, there is only one positive root since $p(0)<0$ and $p(1)>0$. Stated in terms of $T_{p}$, this implies the existence of a unique solution for the measured temperature $T_{p}$ in the interval $T_{1}<T_{p}<T_{2}$, as noted in the previous subsection $\left[T_{1}\right.$ has been set to zero in Eq. (3.37)]. The sign of $p(x)$ essentially tells us the direction of heat flow for a temperature bias of the probe with respect to its equilibrium value. $p(x)<0(p(x)>0)$ corresponds to heat flowing into (out of) the probe, since we changed the sign in re-arranging Eq. (3.36) to Eq. (3.37). The monotonicity of $p(x)$ is therefore equivalent to the Clausius statement of the second law of thermodynamics, and also ensures the uniqueness of the temperature measurement. Such statements of the second law were explored in great detail in chapter 2 . 


\subsection{Numerical Results and Discussion}

In order to illustrate the above theoretical results, and further characterize the local properties of the nonequilibrium steady state, we now present numerical calculations for several molecular junctions with $\pi$-conjugation. In all of the simulations, the molecule is connected to a cold reservoir $R 1$ at $T_{1}=0 \mathrm{~K}$ and a hot reservoir $R 2$ at $T_{2}=100 \mathrm{~K}$. There is no electrical bias; both electrodes have chemical potential $\mu_{0}$. The temperature probe is modeled as an atomically-sharp Au tip scanned horizontally at a constant vertical height of $3.5 \AA$ above the plane of the carbon nuclei in the molecule (tunneling regime). The figures figs. 3.1 to 3.3 appear at the end of this chapter.

The molecular Hamiltonian is described within Hückel theory, $H_{\mathrm{mol}}=$ $\sum_{<i, j>} t_{i j} d_{i}^{\dagger} d_{j}+$ h.c, with nearest-neighbor hopping matrix element $t=-2.7 \mathrm{eV}$. The coupling of the molecule with the reservoirs is described by the tunnelingwidth matrices $\Gamma^{\alpha}$. The retarded Green's function of the junction is given by $G^{r}(\omega)=\left[\mathrm{S} \omega-H_{\mathrm{mol}}-\Sigma_{T}(\omega)\right]^{-1}$, where $\Sigma_{T}=-i \sum_{\alpha} \Gamma^{\alpha} / 2$ is the tunneling self-energy. We take the lead-molecule couplings in the broad-band limit, i.e., $\Gamma_{n m}^{\alpha}(\omega)=\Gamma_{n m}^{\alpha}\left(\mu_{0}\right)$ where $\mu_{0}$ is the Fermi energy of the metal leads. We also take the lead-molecule couplings to be diagonal matrices $\Gamma_{n m}^{\alpha}(\omega)=\Gamma_{\alpha} \delta_{n l} \delta_{m l}$ coupled to a single $\pi$-orbital $l$ of the molecule. $\mathbb{S}$ is the overlap-matrix between the atomic orbitals on different sites and we take $\mathbb{S}=\mathbb{1}$, i.e., an orthonormal set of atomic orbitals. The leadmolecule couplings are taken to be symmetric, with $\Gamma_{1}=\Gamma_{2}=0.5 \mathrm{eV}$. The non-zero elements of the system-reservoir couplings for $R 1$ (cold) and $R 2$ (hot) are indicated with a blue and red square, respectively, corresponding to the carbon atoms in the molecule covalently bonded to the reservoirs. The tunneling-width matrix $\Gamma^{p}$ describing probe-sample coupling is also treated in the broad-band limit (see section 3.2 .2 for details pertaining to the modeling of probe-system coupling). The probe is in the tunneling regime and the probe-system coupling is weak (few $\mathrm{meV}$ ) in comparison to the system-reservoir couplings $\left(\Gamma_{1}=\Gamma_{2}=0.5 \mathrm{eV}\right)$.

It must be emphasized that, although we take a noninteracting Hamiltonian for 
the isolated molecule, our results depend only upon the existence of transmission nodes in the elastic transport regime, which are a characteristic feature of coherent quantum transport, and do not depend on the particular form of the junction Hamiltonian.

\subsubsection{Local Temperatures}

We considered several different molecules and electrode configurations, with and without transmission nodes, and searched for the coldest spot in each system (indicated by a green dot in the figures) as measured by the scanning thermoelectric probe.

The local temperature $T_{p}(\mathbf{x})$ depends on the transmissions from the reservoirs into the probe [Eq. (3.14)], determined by the local probe-system coupling $\Gamma^{p}(\mathbf{x})$ (see section 3.2.2), and is thus a function of probe position. The coldest spot was found using a particle swarm optimization technique that minimizes the ratio of the transmissions to the probe (within a thermal window) from the hot reservoir $R 2$ to that of the cold reservoir $R 1$, within a search space that spans the $z$-plane at $3.5 \AA$ and restricted in the $x y$ direction to within $1 \AA$ from the edge of the molecule甲 The numerical solution to Eq. (3.3) was found using Newton's method. While the algorithm was found to converge rapidly for most points (less than 15 iterations), it is still computationally intensive since the evaluation of the currents given by Eq. (3.13) must have sufficient numerical accuracy. We also note that the minimum probe temperature obtained for each junction does not depend strongly on the distance between the plane of the scanning probe and that of the molecule. This is explained as follows: the probe temperature must depend upon the relative magnitudes of the transmissions into the probe from the two reservoirs and not their actual values. Therefore, the temperature remains roughly independent of the coupling strength $\operatorname{Tr}\left\{\Gamma^{p}\right\}$. It has also been previously noted in Ref. [30] that the local temperature

\footnotetext{
${ }^{\dagger}$ It is necessary to minimize the ratio of transmissions to find the temperature minima since it is computationally prohibitive to calculate the temperatures at various points in the search space, within each iteration of the optimization algorithm
} 


\begin{tabular}{|c|c|c|c|}
\hline Junction & $\begin{array}{c}T_{p}[K] \\
\text { Numerical }\end{array}$ & $\begin{array}{c}T_{p}[K] \\
\text { Analytic }\end{array}$ & Eq. \\
\hline benzene (meta) & 0.154 & 0.1526 & 3.31 \\
\hline benzene (para) & 4.624 & 4.627 & 3.24 \\
\hline pyrene & 0.0821 & 0.0817 & 3.31 \\
\hline coronene & 0.0349 & 0.0355 & $(3.31)$ \\
\hline
\end{tabular}

Table 3.1: The table shows the lowest temperatures found in the different junctions considered. All junctions have the same bias conditions: $T_{1}=0 \mathrm{~K}$, $T_{2}=100 \mathrm{~K}$ and no electrical bias. The right-most column shows the equation used to compute the temperature analytically. We obtain excellent agreement between the numerical and analytic results. The para configuration of the benzene junction does not display a node in the probe transmission spectrum and therefore the minimum probe temperature is not strongly suppressed.

measurement showed little change with the coupling strength even when varied over several orders of magnitude. The restrictions placed on our search space within the optimization algorithm are therefore well justified.

Fig. 3.1 shows the temperature distribution for two configurations of Au-benzene$\mathrm{Au}$ junctions with the chemical potentials of the metal leads at the middle of the HOMO-LUMO gap. The mid-gap region is advantageous since (i) the molecule is charge neutral when the lead chemical potentials are tuned to the mid-gap energy and (ii) the mismatch between the metal leads' Fermi energy and the mid-gap energy is typically small (less than $1-2 \mathrm{eV}$ for most metal-molecule junctions) and available gating techniques [106] would be sufficient to tune across the gap. In both junctions, the region of lowest temperature passes through the two sites in meta orientation relative to the hot electrode because the transmission probability from the hot electrode into the probe is minimum when it is at these locations [63]. The meta junction exhibits much lower minimum temperature measurements since there is a transmission node from the hot reservoir $R 2$ at the mid-gap energy but such nodes are absent in the para junction. Table 3.1 shows the coldest temperature found in each of the junctions presented here.

We only present two junction geometries for benzene here to illustrate that the existence of a node in the probe transmission spectrum, at the mid-gap energy, 
depends upon the junction geometry. The nodes are also absent in the ortho configuration of benzene and the lowest temperature found in this case is similar to that found in the para configuration.

Fig. 3.2 shows the temperature distribution in a gated Au-pyrene-Au junction, and the transmissions into the probe from the two reservoirs at the coldest spot. In general, nodes in the transmission spectrum occur only in a few of the possible junction geometries. As in the benzene junctions, the coldest regions in the pyrene junction pass through the sites to which electron transfer from the hot electrode is blocked by the rules of covalence [63] describing bonding in $\pi$-conjugated systems. We note that the temperature distribution shown in Fig. 3.2 differs significantly from that shown in Ref. [63] for four important reasons: (i) the junction configuration in Fig. 3.2 is asymmetric while that considered in Ref. [63] was symmetric; (ii) the thermal coupling $\kappa_{p 0}$ of the temperature probe to the ambient environment has been set to zero in Fig. 3.2 to allow for resolution of temperatures very close to absolute zero, while the probe in Ref. [63] was taken to have $\kappa_{p 0}=10^{-4} \kappa_{p 0}$, where $\kappa_{p 0}=\left(\pi^{2} / 3\right)\left(k_{B}^{2} T / h\right)=2.84 \times 10^{-10} \mathrm{~W} / \mathrm{K}$ at $T=300 \mathrm{~K}$ is the thermal conductance quantum [107, 108]; (iii) the transport in Fig. 3.2 is assumed to take place at the mid-gap energy due to appropriate gating of the junction, while Ref. 63] considered a junction without gating; and (iv) Ref. 63] considered temperature measurements only in the linear response regime while the thermal bias applied in Fig. 3.2 is essentially outside the scope of linear response. Points (ii)-(iv) also differentiate the results for benzene junctions shown in Fig. 3.1 from the linear-response results of Ref. 63.

Fig. 3.3 shows the temperature distribution in a gated Au-coronene-Au junction exhibiting a node in the probe transmission spectrum. The junction shown was one of three such geometries to exhibit nodes (10 distinct junction geometries were considered). Again, the coldest regions in the junction pass through the sites to which electron transfer from the hot electrode is blocked by the rules of covalence. The coronene junction in Fig. 3.3 displays the lowest temperature amongst all the different junctions considered, with a minimum temperature of $T_{p}=35 \mathrm{mK}$. It should 
be noted that this temperature would be suppressed by a factor of 100, i.e., $T_{p}=$ $350 \mu \mathrm{K}$ if $R 2$ were held at $10 \mathrm{~K}$ due to the quadratic scaling of $T_{p}$ with respect to the temperature $T_{2}$ [cf. Eq. (3.31)]. Higher-order nodes would produce even greater suppression.

\subsubsection{Model of Probe-Sample Coupling}

The scanning thermoelectric probe is modeled as an atomically sharp Au tip operating in the tunneling regime. The probe tunneling-width matrice $f^{f}$ may be described in general as $\Gamma_{n m}^{p}(\omega)=2 \pi V_{n} V_{m}^{*} \rho_{p}(\omega)$, where $\rho_{p}(\omega)$ is the local density of states of the apex atom in the probe electrode and $V_{m}, V_{n}$ are the tunneling matrix elements between the quasi-atomic wavefunctions of the apex atom in the electrode and the $m^{t h}, n^{t h} \pi$-orbitals in the molecule. We consider the Au tip to be dominated by the s-orbital character and neglect all other contributions. The probe-system coupling is also treated within the broadband approximation. The tunneling-width matrix $\Gamma^{p}$ describing the probe-system coupling is in general non-diagonal and is calculated using the methods highlighted in Ref. [16].

\subsection{Conclusions}

We asked a question motivated by the third law of thermodynamics: Is it possible to measure a local temperature of absolute zero in a nonequilibrium quantum system? A simple analysis of this question immedietely tells us that the answer is no, consistent with the third law of thermodynamics. We noted then that voltage biases near zero temperature necessarily lead to heating. We therefore investigated local electronic temperature distributions in nanoscale quantum conductors with one of the reservoirs held at finite temperature and the other held at or near absolute zero, a problem essentially outside the scope of linear response theory.

We obtained local temperatures close to absolute zero when electrons originating from the finite temperature reservoir undergo destructive quantum interference.

\footnotetext{
${ }^{\ddagger}$ I would like to thank Justin Bergfield for modeling the tip-sample coupling.
} 
The local temperature was computed by numerically solving a nonlinear system of equations [Eqs. (3.3) and (3.13)] describing equilibration of a scanning thermoelectric probe with the system, and we obtain excellent agreement with analytic results [Eqs. 3.24), 3.31), and (3.39)] derived using the Sommerfeld expansion. Our conclusion is that a local temperature equal to absolute zero is impossible in a nonequilibrium quantum system but arbitrarily low finite values are, in principle, possible. 

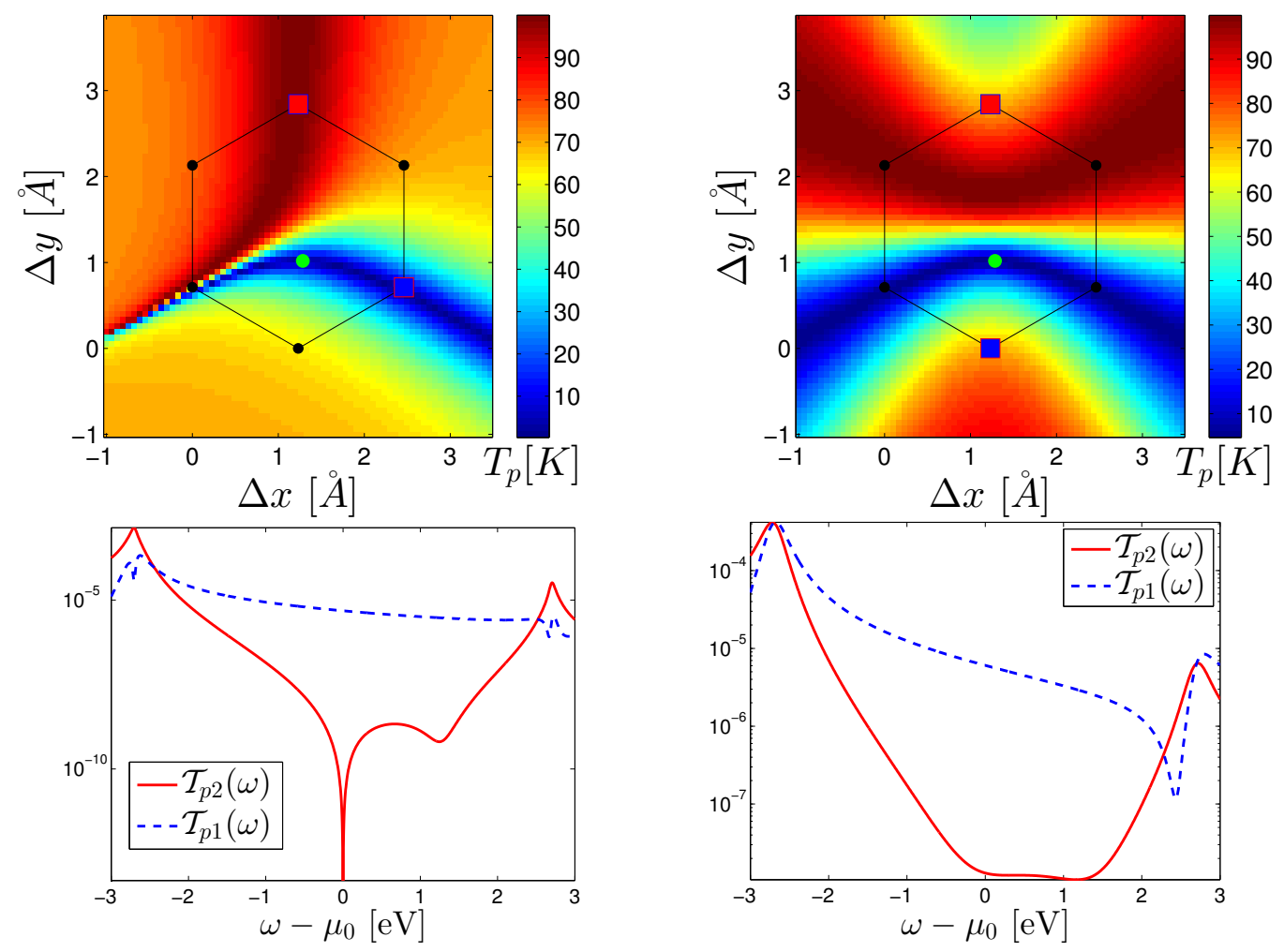

Figure 3.1: Upper panels: Local temperature distributions for Au-benzene-Au junctions in meta and para configurations, respectively. The thermal bias is supplied by cold $\left(T_{1}=0 \mathrm{~K}\right)$ and hot $\left(T_{2}=100 \mathrm{~K}\right)$ reservoirs covalently bonded to the atoms indicated by the blue and red squares, respectively. There is no electrical bias. The probe is scanned at a height of $3.5 \AA$ above the plane of the carbon nuclei in the molecule. The green dots shown in the temperature distributions correspond to the coldest temperature found in each of the junction configurations. Bottom panels: Transmission probabilities into the probe from $R 1$ (cold, i.e., blue curve) and $R 2$ (hot, i.e., red curve), when the probe is positioned over the coldest spot (shown by the corresponding green dot in the upper panel). A transmission node in the meta configuration leads to a greatly suppressed probe temperature [cf. table 3.1]. Note the very different vertical scales in the bottom panels. 

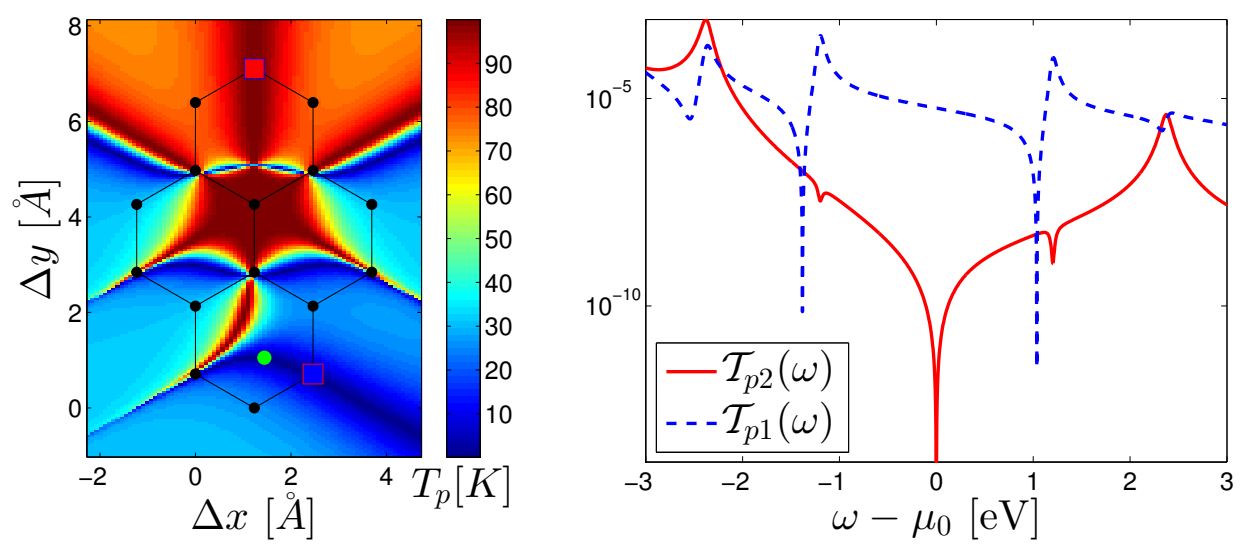

Figure 3.2: Left panel: Probe temperature distribution in a Au-pyrene-Au junction under the same conditions described in Fig. 3.1. The green dot corresponds to the coldest temperature found by the search algorithm. Right panel: Transmissions into the probe from the hot reservoir $R 2$ (red) and the cold reservoir $R 1$ (blue) at the coldest position, indicated by the green dot on the left. The probe transmission from $R 2$ exhibits a (mid-gap) node at the Fermi energy $\mu_{0}$ of the reservoirs, thereby suppressing the temperature measured by the probe. 

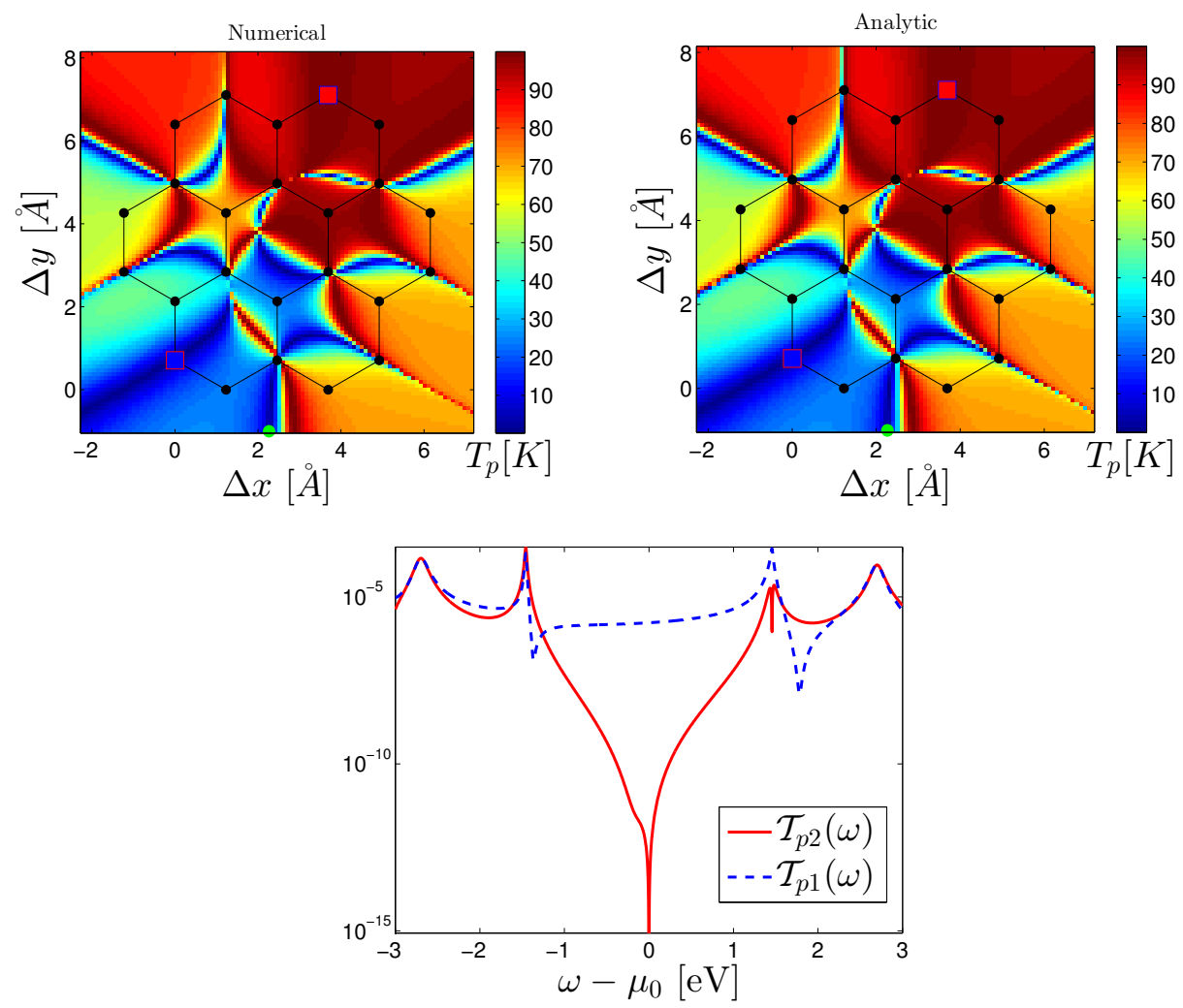

Figure 3.3: Upper panels: Probe temperature distributions for a Au-coronene-Au junction under the same conditions described in Fig. 3.1. The numerically calculated temperature is on the left, and to the right is the analytically calculated temperature using Eq. (3.24). Although it is in excellent qualitative agreement (and quantitative agreement for the most part), Eq. (3.24) poorly estimates the temperature for the coldest spot (shown in green) due to the existence of a transmission node. Eq. (3.31) gives the correct estimation in the presence of a node, while Eq. (3.24) incorrectly predicts $T_{p}=0 \mathrm{~K}$. The lower panel shows the probe transmissions from the two reservoirs $\left(R 1\right.$ with $T_{1}=0 \mathrm{~K}$ in blue and $R 2$ with $T_{2}=100 \mathrm{~K}$ in red) corresponding to the probe positioned over the coldest spot (shown in green). 


\section{CHAPTER 4}

STM as a Thermometer

This chapter is yet to be published.

In chapter 2 we showed that a measurement of temperature has to be accompanied with a measurement of voltage as well. We discuss here the experimental consequences of the results of chapter 2 .

\subsection{Introduction}

Thermal imaging of nanoscale systems is of crucial importance not only due to its potential applications in future technologies, but also because it can greatly enhance our understanding of heat transport at the smallest scales. In recent years, nanoscale thermometry has found application in a wide range of fields including thermometry in a living cell [109], local control of chemical reactions [110] and temperature mapping of operating electronic devices [111]. Various studies utilize radiationbased techniques such as Raman spectroscopy [112], fluorescence in nanodiamonds [109, 113] and near-field optical microscopy [114]. The spatial resolution of these radiation-based techniques are limited due to optical diffraction and, to overcome this drawback, scanning probe techniques [115] have seen a flurry of activity in recent years [54, 116, 117]. Despite the remarkable progress made by scanning probe thermometry, the spatial resolution remains in the 10 -nm range.

Since temperature and voltage are both fundamental thermodynamic observables, it is instructive to draw the sharp contrast that exists between the measurement of these two quantities at the nanoscale. Scanning probe potentiometry (STP) [18] is a mature technology and can map local voltage variations with sub-angstrom

spatial resolution by operating in the tunneling regime. STP has been used to map the local voltage variations in the vicinity of individual scatterers, interfaces 
or boundaries [118 122], providing direct observations of the Landauer dipole [123]. STP has been a useful tool in distangling the different scattering mechanisms [122], and can map local potential variations due to quantum interference effects [120, 121]. Similarly, temperature oscillations due to quantum interference effects have been theoretically predicted for various nanosystems out of equilibrium [30, 57, 63, 64. but have hitherto remained outside the reach of experiment.

Scanning thermal microscopy (SThM) relies on the measurement of a heat-flux related signal that can be sensed based on a calibrated thermocouple or electrical resistor [115]. A good thermal contact between the tip and the sample is needed for an appreciable heat-flux and generally implies a measurement in the contact regime, thus limiting the spatial resolution. Previous studies have addressed various technical difficulties in the measurement such as the parasitic heat transfer through air and the loss of spatial resolution due to the formation of a liquid meniscus at the tip-sample interface [52]. A dramatic enhancement in the spatial resolution was later reported by operating the probe in an ultra high vacuum (UHV) environment [54], allowing quantitative thermometry with $\sim 10 \mathrm{~nm}$ spatial resolution. SThM measurements are typically affected by contact related artifacts, most importantly by an unknown tip-sample thermal resistance which previous studies have attempted to mitigate [124]. Additionally, in SThM measurements on systems out of equilibrium, the tip-sample thermal conductance must be large compared to the probe-cantilever conductance which is not easy to achieve. A further concern is that the resulting operation would be invasive (due to the strong thermal coupling between the probe and sample) has also been pointed out. We encourage the reader to refer to Ref. [124] for a recent review and extensive reference list. Recently a significant advance was reported [117] by simultaneously probing the time-modulated and time-averaged heat-fluxes on a sample driven out of equilibrium by a time-modulated voltage resulting in a spatial resolution of $\sim 7 \mathrm{~nm}$.

Here we propose a method to overcome the most daunting technical challenges of SThM by simultaneously measuring the conductance and thermopower. We predict a dramatic increase in the spatial resolution of more than two orders of magnitude 
allowing experiments to probe longstanding theoretical predictions on quantum coherent conductors. The method requires standard scanning tunneling microscopy (STM) techniques whose operation in the tunneling regime accounts for the dramatic increase in the spatial resolution. Our method is applicable to any quantum coherent conductor which encompasses systems from the mesoscopic scale to single molecules. The central assumption for such measurements is the validity of the Wiedemann-Franz (WF) law. The WF law is the statement of a rather simple observation about heat transport in electrical conductors. It asserts that in electrical conductors, heat is carried mainly by the electrons and that therefore electrical $(\sigma)$ and thermal conductance $(\kappa)$ must be related in a material-independent way $\kappa=\sigma L T$, where $L=\pi^{2} k_{B}^{2} / 3 e^{2}$ is a universal constant and $T$ is the temperature. While its applicability in bulk metals has been known for over 150 years, it can be shown to hold true in quantum systems where transport is dominated by elastic processes (see appendix C, section C.1, for the derivation). Recently, there have been experimental verifications of the WF law in atomic contact junctions [27, 28].

\subsection{Temperature Measurements}

In operating nanoscale devices, it is quite clear that the voltage refers to the electronic voltage since they are the only charged species participating in the transport. However, it is less clear what is meant by a temperature in this context since heat can be carried by other degrees of freedom such as phonons and photons as well. Out of equilibrium, the temperatures of the different degrees of freedom do not coincide. A majority of nanoscale electronic devices operate in the elastic transport regime where electron and phonon degrees of freedom are completely decoupled, and the distinction between their temperatures become extremely important. SThM measurements cannot make this distinction since they rely on measuring heat-flux related signals which carry contributions from all degrees of freedom. Our method provides the much needed characterization of the electronic temperature, decoupled from all other degrees of freedom, thereby providing a vital tool to characterize 
nonequilibrium device performance. In the remainder of the chapter, we refer only to the electronic temperature.

We note a crucial, but often overlooked, theoretical point pertaining to the imaging of temperature fields on a nonequilibrium conductor. The prevailing paradigm for temperature and voltage measurements is the following [56]: (i) a voltage is measured by a probe (voltmeter/thermometer) when in electrical equilibrium and (ii) a temperature is measured when in thermal equilibrium with the sample. We refer to this definition as the Engquist-Anderson (EA) definition. The fact that the EA definition implicitly ignores thermoelectric effects was pointed out by Bergfield and Stafford [63], and a notion of a joint probe was put forth by requiring both electrical and thermal equilibrium with the sample. A temperature probe without electrical equilibrium develops a temperature bias at the probe-sample junction due to the Peltier effect. Similarly, a voltage probe without thermal equilibrium develops a voltage bias at the probe-sample junction due to the Seebeck effect. These errors can be quite large in quantum coherent conductors [74]. A temperature probe therefore has to remain in thermal and electrical equilibrium with the nonequilibrium sample [30, 31, 41, 48, 63, 74], thereby ensuring true thermodynamic equilibrium of the measurement apparatus with the nonequilibrium sample.

The joint probe measurement was made mathematically rigorous in chapter 2 where it was shown that the probe equilibration always exists and is unique, arbitrarily far from equilibrium and with arbitrary interactions within the quantum system. Moreover, it was shown that the EA definition is provably nonunique: The value measured by the EA thermometer depends quite strongly on its voltage and, conversely, the value measured by the EA voltmeter depends on its temperature. These results are deeply connected to the second law of thermodynamics and expose the fatal flaw in the EA definition. A measurement of electron temperature therefore has to involve also a simultaneous measurement of voltage simply due to the fact that electrons carry both charge and heat. Therefore we may write

$$
I_{p}=J_{p}=0
$$


for the simultaneous vanishing of the particle current $I_{p}$ and the electronic contribution to the heat current $J_{p}$.

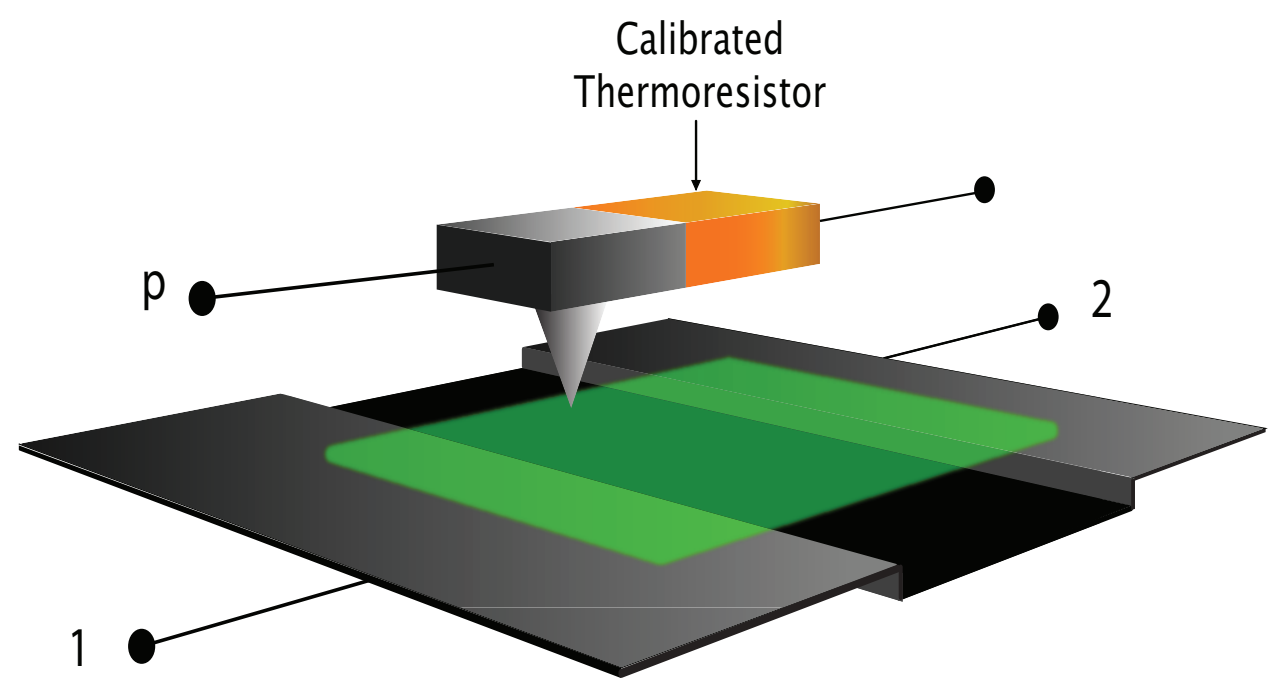

Figure 4.1: A schematic depiction of the measurement apparatus. The nanostructure (shown in green) is on an interconnect structure (shown in grey) which is connected to macroscopic reservoirs 1 and 2 which drives the system out of equilibrium using a voltage and/or temperature bias. The temperature and voltage must be simultaneously measured to ensure a unique measurement. The thermopower measurement uses a calibrated thermoresistor in series with the scanning tunneling tip which is in contact with the macroscopic probe reservoir p. Simultaneous measurement of the conductance and thermopower determines the temperature and voltage in the linear response regime. Measurements made in the tunneling regime result in a dramatic increase of the spatial resolution.

\subsection{Temperature via Electrical Measurement}

The measurement operation involves an STM tip scanning the surface of the conductor at a constant height in the tunneling regime as shown in Fig. 4.1. We propose to measure simultaneously the conductance and thermopower by inducing timemodulated changes in the probe voltage and temperature, respectively. Since the bias voltages or temperatures cannot exceed $k_{B} T_{0}$ for operation within the linear response regime, we do not envision such measurements at liquid He temperatures and present our calculations at an equilibrium temperature of $T_{0}=100 \mathrm{~K}$. Our method 
is therefore fully compatible with standard lock-in techniques so as to overcome the noise due thermal fluctuations at finite operating temperatures. Terminals 1 and 2 in Fig. 4.1 represent macroscopic reservoirs attached to the interconnect, while p labels the macroscopic reservior connected to the STM tip. The nanostructure (shown in green) together with the interconnect structure (shown in grey) forms the central system. The resulting open quantum system is driven out of equilibrium by a bias of the terminals 1 and 2, while the probe is noninvasive due to operation in the tunneling regime. The conductance is measured by the application of a timemodulated voltage at the probe tip and the resulting current in the macroscopic contacts is recorded. Similarly, the thermopower is measured by the application of a time-modulated temperature signal at the probe tip. The thermopower measurement therefore requires a calibrated thermoresistor in series with the probe. Although simultaneous measurements of the conductance and thermopower have been made on single molecules using STM-based break junctions [21, their relevance as a joint probe for temperature and voltage in nonequilibrium systems has remained entirely unappreciated.

Within the linear response regime, the probe currents depend linearly on the temperature and voltage gradients

$$
\left(\begin{array}{c}
I_{p} \\
J_{p}
\end{array}\right)=\sum_{\alpha}\left(\begin{array}{cc}
\mathcal{L}_{p \alpha}^{(0)} & \mathcal{L}_{p \alpha}^{(1)} \\
\mathcal{L}_{p \alpha}^{(1)} & \mathcal{L}_{p \alpha}^{(2)}
\end{array}\right)\left(\begin{array}{c}
\mu_{\alpha}-\mu_{p} \\
\frac{T_{\alpha}-T_{p}}{T_{0}}
\end{array}\right)
$$

where $\mathcal{L}_{p \alpha}^{(\nu)}$ are the Onsager linear response coefficients evaluated at the equilibrium temperature $T_{0}$ and chemical potential $\mu_{0}$. They are related to the electrical conductance, thermopower and thermal conductance of the probe-reservoir junction

$$
\begin{aligned}
G_{p \alpha}\left(\mu_{0}, T_{0}\right) & =e^{2} \mathcal{L}_{p \alpha}^{(0)}\left(\mu_{0}, T_{0}\right) \\
S_{p \alpha}\left(\mu_{0}, T_{0}\right) & =-\frac{\mathcal{L}_{p \alpha}^{(1)}\left(\mu_{0}, T_{0}\right)}{e T_{0} \mathcal{L}_{p \alpha}^{(0)}\left(\mu_{0}, T_{0}\right)} \\
\kappa_{p \alpha}\left(\mu_{0}, T_{0}\right) & =\frac{1}{T_{0}}\left(\mathcal{L}_{p \alpha}^{(2)}\left(\mu_{0}, T_{0}\right)-\frac{\left(\mathcal{L}_{p \alpha}^{(1)}\right)^{2}}{\mathcal{L}_{p \alpha}^{(0)}}\right) .
\end{aligned}
$$


The co-efficients $\mathcal{L}_{p \alpha}^{(0)}$ and $\mathcal{L}_{p \alpha}^{(1)}$ are accessible via electrical measurement whereas

$$
\mathcal{L}_{p \alpha}^{(2)}\left(\mu_{0}, T_{0}\right)=\frac{\pi^{2}}{3} k_{B}^{2} T_{0}^{2} \mathcal{L}_{p \alpha}^{(0)}
$$

to leading order in the Sommerfeld series. Eq. (4.4) is the Wiedemann-Franz law?

We may write down the exact solution to Eq. (4.1) in the linear response regime 30

$$
\begin{aligned}
\frac{T_{p}^{(\text {Exact })}}{T_{0}} & =\frac{\sum_{\beta} \mathcal{L}_{p \beta}^{(0)} \sum_{\alpha} \mathcal{L}_{p \alpha}^{(1)} \mu_{\alpha}-\sum_{\beta} \mathcal{L}_{p \beta}^{(1)} \sum_{\alpha} \mathcal{L}_{p \alpha}^{(0)} \mu_{\alpha}}{\sum_{\beta} \mathcal{L}_{p \beta}^{(2)} \sum_{\alpha} \mathcal{L}_{p \alpha}^{(0)}-\left(\sum_{\alpha} \mathcal{L}_{p \alpha}^{(1)}\right)^{2}} \\
& +\frac{1}{T_{0}} \frac{\sum_{\beta} \mathcal{L}_{p \beta}^{(0)} \sum_{\alpha} \mathcal{L}_{p \alpha}^{(2)} T_{\alpha}-\sum_{\beta} \mathcal{L}_{p \beta}^{(1)} \sum_{\alpha} \mathcal{L}_{p \alpha}^{(1)} T_{\alpha}}{\sum_{\beta} \mathcal{L}_{p \beta}^{(2)} \sum_{\alpha} \mathcal{L}_{p \alpha}^{(0)}-\left(\sum_{\alpha} \mathcal{L}_{p \alpha}^{(1)}\right)^{2}} .
\end{aligned}
$$

Our method relies on the WF law given by Eq. (4.4), and to leading order in the Sommerfeld expansion we have

$$
\begin{aligned}
\frac{T_{p}^{(\mathrm{WF})}}{T_{0}}= & \frac{3}{\pi^{2} k_{B}^{2} T_{0}^{2}}\left(\frac{\sum_{\alpha} \mathcal{L}_{p \alpha}^{(1)} \mu_{\alpha}}{\sum_{\alpha} \mathcal{L}_{p \alpha}^{(0)}}-\frac{\sum_{\alpha} \mathcal{L}_{p \alpha}^{(1)}}{\sum_{\alpha} \mathcal{L}_{p \alpha}^{(0)}} \frac{\sum_{\beta} \mathcal{L}_{p \beta}^{(0)} \mu_{\beta}}{\sum_{\beta} \mathcal{L}_{p \beta}^{(0)}}\right) \\
& +\frac{\sum_{\alpha} \mathcal{L}_{p \alpha}^{(0)} T_{\alpha}}{T_{0} \sum_{\alpha} \mathcal{L}_{p \alpha}^{(0)}}
\end{aligned}
$$

$T_{p}^{(\mathrm{WF})}$ requires only the measurement of $\mathcal{L}_{p \alpha}^{(0)}$ and $\mathcal{L}_{p \alpha}^{(1)}$ and lends itself to a simple interpretation. The first term in Eq. 4.6) is the thermoelectric contribution whereas the second term is the thermal contribution. It differs from $T_{p}^{(\text {Exact })}$

$$
T_{p}^{(\mathrm{WF})}=T_{p}^{(\text {Exact })}+T_{p}^{(\text {Exact })} \mathcal{O}\left(\left(k_{B} T_{0} / \Delta\right)^{2}\right)
$$

only at the second order of the Sommerfeld series which is typically very small [cf. also Eq. C.15 in appendix C.

The simultaneous measurement of the electrical conductance and thermopower therefore determine both temperature and voltage, since the thermal conductance is simply related to the electrical conductance by the WF law. The method thus offers two distinct advantages over existing SThM schemes: (i) significantly enhanced

*The Wiedemann-Franz law can be derived explicitly for elastic transport as shown in appendix C. section C.1. 

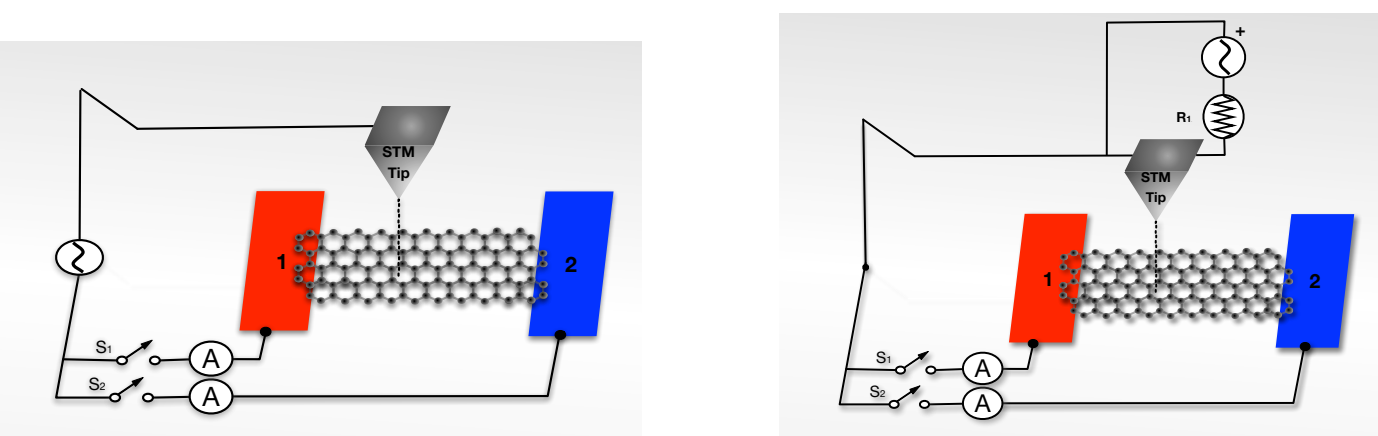

Figure 4.2: The conductance and thermopower, or equivalently the $\mathcal{L}_{p \alpha}^{(0)}$ and $\mathcal{L}_{p \alpha}^{(1)}$ coefficients, can be measured simultaneously by toggling between the two circuits above. The $\mathcal{L}_{p \alpha}^{(0)}$ coefficient is measured by applying a time-modulated voltage at the probe reservoir and recording the resulting current at the reservoir $\alpha$ while all other reservoirs are disconnected using switches, as shown on the left panel. $\mathcal{L}_{p 1}^{(0)}$ for example is measured with S1 ON and S2 OFF. The $\mathcal{L}_{p \alpha}^{(1)}$ coefficient is measured by inducing a time-modulated temperature variation in the probe and recording the current in reservoir $\alpha$ while all other reservoirs are disconnected using switches, as shown on the right panel.

spatial resolution due to measurement in the tunneling regime and (ii) the WF law relates precisely the electronic contribution to the heat flow, thereby providing a much needed tool to characterize the electronic temperature decoupled from all other degrees of freedom. Knowing separately the electronic contribution to heat current is vital for the characterization of nonequilibrium device performance where electrons are the working fluid.

Temperature and voltage measurements on the system are equivalent to the determination of $\mathcal{L}_{p \alpha}^{(0)}$ and $\mathcal{L}_{p \alpha}^{(1)}$ (equivalently conductance and thermopower) and can be measured simultaneously by simply toggling between two circuit operations shown in Fig. 4.21]. In the first circuit, the $\mathcal{L}_{p \alpha}^{(0)}$ coefficient is measured by the application of an ac voltage bias $V(\omega)=V_{p}(\omega)-V_{\alpha}$ across the probe and reservoir $\alpha$ and the current is measured at frequency $\omega$ using a lock-in amplifier

$$
\begin{aligned}
I_{\alpha} & =\mathcal{L}_{p \alpha}^{(0)}\left(\mu_{p}-\mu_{\alpha}\right)=-I_{p}, \\
I_{\alpha}(\omega) & =e \mathcal{L}_{p \alpha}^{(0)} V(\omega) .
\end{aligned}
$$

\footnotetext{
${ }^{\dagger}$ I would like to thank Sosuke Inui for assistance in producing Figs. 4.1 and 4.2
} 

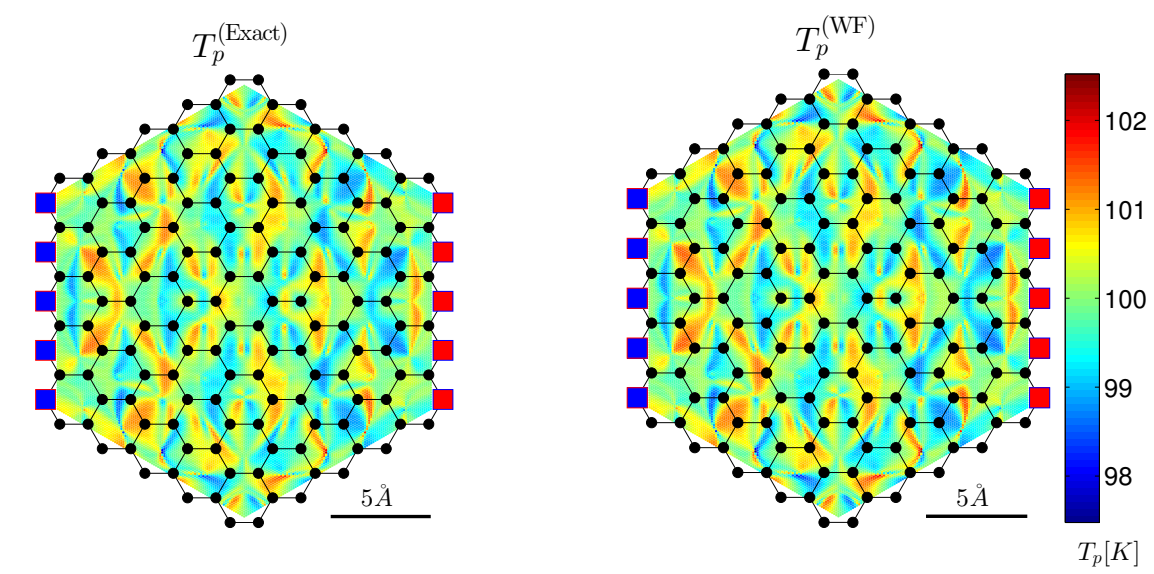

Figure 4.3: Temperature variations on a hexagonal graphene flake with the application of a voltage bias $V_{\text {blue }}-V_{\text {red }}=5 \mathrm{mV}$, where the red and blue squares indicate the sites coupled to the interconnect. The left panel shows the exact linear response solution given in Eq. 4.5) while the right panel shows the approximate solution obtained by employing the WF law given in Eq. (4.6). The discrepancies between the two measurements are of higher order in the Sommerfeld series and are very small. In particular, $T_{p}^{(\mathrm{WF})}$ very slightly overestimates the hottest spots and underestimates the coldest spots while the intermediate temperatures agree exceptionally well. The same temperature scale is used for both panels.

A switch is used to toggle between different reservoirs $\alpha$ as shown in Fig. 4.2. The measurement of the $\mathcal{L}_{p \alpha}^{(1)}$ coefficient uses a calibrated thermoresistor in series with the probe. An ac voltage at frequency $\omega$ induces temperature variations at frequency $2 \omega$ due to Joule heating, and the resulting current in reservoir $\alpha$ (at frequency $2 \omega$ ) is then measured

$$
\begin{aligned}
I_{\alpha} & =\mathcal{L}_{p \alpha}^{(1)} \frac{\left(T_{p}-T_{\alpha}\right)}{T_{0}} \\
I_{\alpha}(2 \omega) & =\mathcal{L}_{p \alpha}^{(1)} \frac{T_{p}(2 \omega)}{T_{0}} .
\end{aligned}
$$

The measurements involve changing the probe bias conditions and constitute a noninvasive measurement since the probe is operated in the tunneling regime. Since the resulting currents are affected by the probe-reservoir bias amplitudes, their values must be chosen such that the currents are resolvable. Charge currents of a few hundred pA are quite easily measured with lock-in techniques and our method therefore works well with typical voltage and temperature biases for most conductors. 

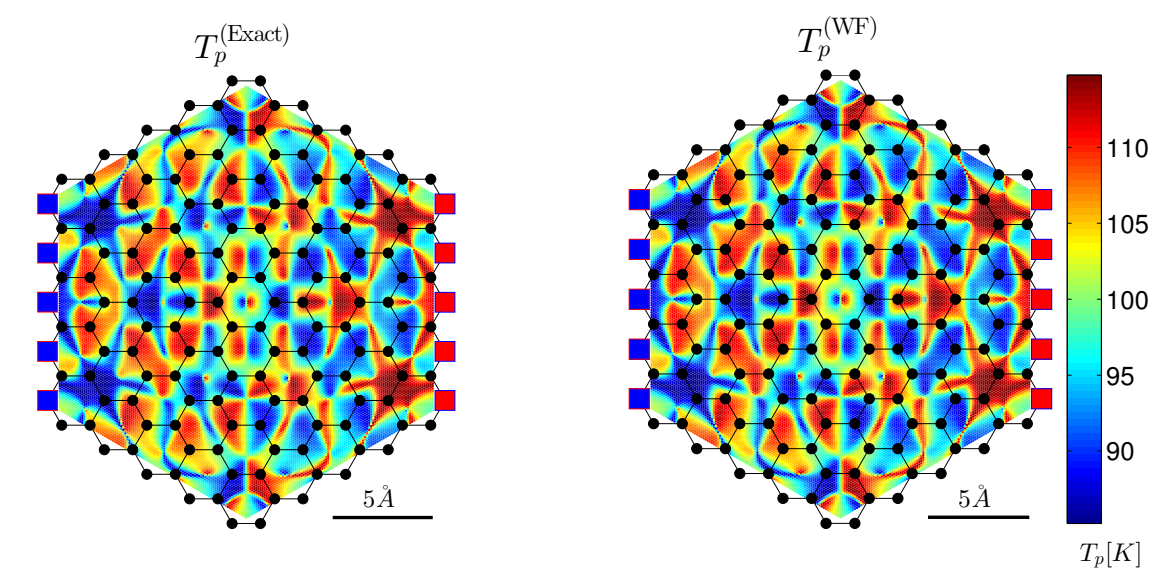

Figure 4.4: Temperature variations on a hexagonal graphene flake with the application of a temperature bias $T_{\text {red }}-T_{\text {blue }}=30 \mathrm{~K}$, where the red and blue squares indicate the sites coupled to the interconnect. The left panel shows the exact linear response solution given in Eq. (4.5) while the right panel shows the WF solution given by Eq. (4.6). The discrepancies between the two measurements are of higher order in the Sommerfeld series and are very small. In particular, $T_{p}^{(\mathrm{WF})}$ very slightly overestimates the hottest spots and underestimates the coldest spots while the intermediate temperatures agree exceptionally well. The same temperature scale is used for both panels.

\subsection{Results}

Fig. 4.3 shows the variations in temperature for a voltage bias of $V_{\text {blue }}-V_{\text {red }}=$ $5 \mathrm{mV}$ at $T_{0}=100 \mathrm{~K}$ for a hexagonal graphene flake. The red and blue squares shown in the figure correspond to the carbon atoms of graphene bonded to the interconnects. Temperature variations $\sim 5 \mathrm{~K}$ are seen within the nanostructure and clearly demonstrate the quantum coherent nature of transport. The agreement between $T_{p}^{(\text {Exact })}$ and $T_{p}^{(\mathrm{WF})}$ given by eqs. 4.5 and 4.6), respectively, is exceptional. However, closer inspection of the figure reveals that the hottest spots recorded in $T_{p}^{(\mathrm{WF})}$ (electrically measurable) are slightly hotter than the ones given by the exact solution $T_{p}^{(\text {Exact })}$. Similarly, the coldest spots in our method are slightly colder than the ones given by the exact solution. Discrepencies are due to higher-order contributions in the Sommerfeld series [cf. Eq. 4.7)] and can be made even smaller by operation at lower temperatures. We note that the temperature variations shown 
here are solely the result of the voltage bias and are given by the first term in Eq. (4.6). Since the variations are purely due to the thermoelectric effect, the EA probe would have noted no temperature variations at all.

Fig. 4.4 shows the variations in temperature for a temperature bias of $T_{\text {red }}-$ $T_{\text {blue }}=30 \mathrm{~K}$ at $T_{0}=100 \mathrm{~K}$ for the same hexagonal graphene flake with identical couplings to the interconnects, shown again by the red and blue squares. The temperature variations $\sim 30 \mathrm{~K}$ clearly demonstrate the nonclassical nature of heat transport at the nanoscale. The agreement between $T_{p}^{(\text {Exact })}$ and $T_{p}^{(\mathrm{WF})}$ given by eqs. (4.5) and (4.6), respectively, is still extremely good. Once again, closer inspection of the figure reveals that the hottest spots recorded in $T_{p}^{(\mathrm{WF})}$ (electrically measurable) are slightly hotter than the ones given by the exact solution $T_{p}^{(\text {Exact })}$. Similarly, the coldest spots in our method are slightly colder than the ones given by the exact solution. Discrepencies are due to higher-order contributions in the Sommerfeld series [cf. Eq. 4.7)] and can be made even smaller by operation at lower temperatures. We note that the temperature variations shown here are solely the result of the temperature bias and are given by the first term in Eq. (4.6). The EA probe gives the correct result for this situation.

\subsubsection{The System Hamiltonian}

We show a hexagonal graphene flake connected to the interconnect at the sites represented by the blue and red squares in figs. 4.3 and 4.4 , and the interconnect is in contact with macroscopic reservoirs whose bias conditions can be adjusted. The strong coupling of the system to the macroscopic reservoirs creates an open quantum system with energy level broadenings, and is described by the tunnelingwidth matrices $\Gamma^{\alpha}$. The Hamiltonian of the graphene flake is described within Hückel theory

$$
H=-\sum_{<i, j>} t_{i j} d_{i}^{\dagger} d_{j}+\text { h.c. }
$$

with nearest-neighbor interaction $t=-2.7 \mathrm{eV}$. The tunneling-width matrix is nonzero only at the sites shown by the blue and red squares and is diagonal in 
the basis of atomic orbitals $\Gamma_{\mathrm{bb}}^{1}=0.5 \mathrm{eV}=\Gamma_{\mathrm{rr}}^{2}$ where $\mathrm{b}$ and $\mathrm{r}$ represent atomic orbitals shown by blue and red squares respectively. The equilibrium chemical potential is set to $\mu_{0}=-1.2 \mathrm{eV}$ with respect to the onsite energy, while the equilibrium temperature $T_{0}=100 \mathrm{~K}$, and the STM tip is scanned at a height of $3.5 \AA$ above the plane of the nanostructure.

\subsection{Conclusions}

We present a method to map temperature variations in nanoscale conductors by using only electrical measurements. The measurements can be made using standard STM techniques and for any conductor where the Wiedemann-Franz law is valid. The method is therefore applicable to the vast majority of operating nanoscale conductors. We elucidate the importance of joint probe measurements which require a vanishing of both the particle and heat currents of electrons entering the probe. The operation of the probe in the tunneling regime results in a dramatic enhancement of the spatial resolution by over two orders of magnitude when compared with existing SThM schemes. Our method precisely accounts for the electronic contribution to the heat current, decoupled from all other degrees of freedom. The method therefore not only provides a way to image quantum coherent temperature variations, but can also be used as a vital tool to characterize nonequilibrium electronic device performance.

\subsection{Note on Experimental Realization}

It was brought to our attention, following discussions with Oliver Monti and Brian LeRoy, that the fabrication of a calibrated thermoresistor on the STM tip is experimentally challenging. However, heating elements can be fabricated in the contact reservoirs which drive the system out of equilibrium, for example, as reported recently in Ref. [125]. Brian LeRoy suggests that a flake of graphene can be used as a heating element since it undergoes Joule heating when sufficient current is passed. Therefore, a flake of graphene, along with a suitable substrate, can be used to induce 
the temperature modulations in the contact reservoirs. This would be an equivalent realization of our experiment since current conservation would imply that the transmission functions satisfy $\mathcal{T}_{p \alpha}=\mathcal{T}_{\alpha p}$. In principle, the experiment can be carried out even in the presence of a magnetic flux $\Phi$ but, since $\mathcal{T}_{p \alpha}(\Phi)=\mathcal{T}_{\alpha p}(-\Phi)$, we would have to invert the magnetic field to infer the $\mathcal{L}_{p \alpha}^{(\nu)}$ coefficients [102]. Furthermore, our initial analyses suggest that the experiment could be performed at cryogenic temperatures as low as 4K: Although the thermoelectric response is suppressed quadratically with decreasing temperature (cf. C.1 for the relevant Sommerfeld series expansion), it is possible to induce larger (experimentally resolvable) electrical currents by an appropriate gating of the system. 


\section{CHAPTER 5}

Entropy

This chapter is based on the work published in Refs. [48, 126] and also contains some work which is yet to be published.

\subsection{Introduction}

Entropy is one of the most fundamental quantities in all of physics. The entropy for any quantum system is given by ${ }^{*}$

$$
S=-\operatorname{Tr}\{\hat{\rho} \log \hat{\rho}\}
$$

Here $\hat{\rho}$ is the density operator describing the quantum system. The log operation is understood in much the same way we understand exponentials of operators. For example, we may write $e^{\hat{A}}=\hat{B}$ and understand that the $l h s$ is a series expansion of operator $\hat{A}$ which converges to $\hat{B}$. Then $\hat{A}=\log \hat{B}$ and is uniquely defined for any density operator. The expression for entropy in the form above was first written by von Neumann [127].

Any density operator can be written in its complele many-body eigenbasis as $\hat{\rho}=\sum_{i} p_{i}\left|\Psi_{i}\right\rangle\left\langle\Psi_{i}\right|$ and the entropy is then easily calculated to be

$$
S=-\sum_{i} p_{i} \log p_{i}
$$

which is a form often encountered in information theory. The $p_{i}$ are the probabilities associated with states $\left|\Psi_{i}\right\rangle$. The density operator has unit trace $\sum_{i} p_{i}=1$. The density operator of a system completely encodes all the statistical information pertaining to the system and is the central quantity in quantum statistical mechanics.

\footnotetext{
${ }^{*}$ It is usual to include a prefactor of $k_{B}$ which we here set to unity.
} 
The statistical average of any operator $\hat{O}$ is $\langle\hat{O}\rangle=\operatorname{Tr}\{\hat{\rho} \hat{O}\}$. One may therefore introduce the Entropy Operator as

$$
\hat{S}=-\log \hat{\rho}
$$

so that the entropy in Eq. (5.1) is the statistical average of the above operator.

It is worth noting that entropy is invariant under unitary transformations of the density operator $\hat{\rho} \rightarrow \hat{U}^{\dagger} \hat{\rho} \hat{U}$. Let us take $\log \hat{\rho}=-\hat{S}=\hat{A}$ so that $\hat{\rho}=e^{\hat{A}}$. Then

$$
\begin{aligned}
\hat{U}^{\dagger} \hat{\rho} \hat{U} & =\hat{U}^{\dagger}\left(1+\hat{A}+\frac{1}{2 !} \hat{A}^{2}+\ldots\right) \hat{U} \\
& =1+\hat{U}^{\dagger} \hat{A} \hat{U}+\frac{1}{2 !} \hat{U}^{\dagger} \hat{A} \hat{U} \hat{U}^{\dagger} \hat{A} \hat{U}+\ldots \\
& =\exp \left(\hat{U}^{\dagger} \hat{A} \hat{U}\right) .
\end{aligned}
$$

This implies that $\log \left(\hat{U}^{\dagger} \hat{\rho} \hat{U}\right)=\hat{U}^{\dagger} \log \hat{\rho} \hat{U}$ and the entropy

$$
\begin{aligned}
S & =-\operatorname{Tr}\left\{\hat{U}^{\dagger} \hat{\rho} \hat{U} \log \left(\hat{U}^{\dagger} \hat{\rho} \hat{U}\right)\right\} \\
& =-\operatorname{Tr}\left\{\hat{U}^{\dagger} \hat{\rho} \hat{U} \hat{U}^{\dagger} \log \hat{\rho} \hat{U}\right\} \\
& =-\operatorname{Tr}\{\hat{\rho} \log \hat{\rho}\} .
\end{aligned}
$$

This means that a closed quantum system evolving under the Schrödinger equation will always have the same entropy. It is only measurements or interactions with an environment which can bring about a change in the system's entropy.

In the remainder of this chapter, we shall discuss entropy in the context of the scattering problem. In such problems, we have a few reservoirs which exchange particles and energy with each other through a central scattering region but the reservoirs themselves are taken to be in the thermodynamic limit. This means that the exchange of particles and energy does not alter the distribution within the reservoirs. The distribution of the reservoirs set the boundary conditions for the scattering problem. The distribution of the reservoirs (their density operator) is completely specified by their temperature and chemical potential since they are taken to be ideal fermi gases. 
The work presented here deals with noninteracting fermions. We formulate the entropy in terms of the scattering states and find that, in the scattering basis, the total entropy is additive over the different reservoirs since their scattering states do not mix in the absence of two-body interactions. We then formulate the entropy that is measured by a local observer who has no knowledge whatsoever of the scattering states but has detailed knowledge of the local spectrum and local distribution function. We find that this lack of knowledge increases the observed entropy. Finally, we formulate the entropy that is measured without knowledge of the local distribution function but knowledge of the local mean particle number and energy. We show that the entropies formulated in the three different ways satisfy a hierarchy of inequalities with the most knowledgable formulation leading to the least entropy. We illustrate our results for a two-level system far from equilibrium. We propose an ansatz for the entropy when two-body interactions are present. Based on the inequalities developed, we also use the entropy as a metric to quantify the "distance" from equilibrium. We also show numerical results for a molecular junction driven far from equilibrium.

\subsubsection{Single Fermionic Level}

We start our discussion by writing down the density operator of a single fermionic level. We do this in the full many-body Hilbert space. Of course, the full manybody Hilbert space for a single fermi level has only two linearly independent state vectors available to it. This would be the vacuum and the one-particle level. The Pauli principle restricts us from having more than one fermion in the same state. Nevertheless, this problem is instructive and can be easily generalized to include more states.

In general, the many-body Hilbert space that is accessible to systems of identical particles is a small subspace of the entire many-body Hilbert space. This is because symmetry constrains the state vectors that are accessible to identical particles obeying a symmetry principle. The allowed state vectors are either symmetric

(bosons) or antisymmetric (fermions) with respect to exchange of any two particles. 
The natural framework to describe a system of identical particles is called second quantization. In the second quantization framework, we do not have to worry about the symmetry principles for the state vectors themselves but rather assume that the entire many-body Hilbert space accessible to them. The symmetry principle is then relegated to the creation and destruction operators which obey a certain commutation relation depending upon the type of symmetry (Fermi or Bose). This leads to a great simplification. The reader may refer to any standard textbook on quantum mechanics for an exposition to this subject.

In equilibrium, the density operator is written as

$$
\hat{\rho}=\frac{e^{-\beta(\hat{H}-\mu \hat{N})}}{\operatorname{Tr}\left\{e^{-\beta(\hat{H}-\mu \hat{N})}\right\}}
$$

where $\beta$ is the inverse temperature and $\mu$ is the chemical potential. The single level Hamiltonian and number operators are

$$
\begin{aligned}
& \hat{H}=\epsilon \hat{\Psi}^{\dagger} \hat{\Psi} \\
& \hat{N}=\hat{\Psi}^{\dagger} \hat{\Psi},
\end{aligned}
$$

where $\hat{\Psi}^{\dagger}$ and $\hat{\Psi}$ are the creation and annihilation operators respectively for the single level with energy $\epsilon$. We write the operator $\hat{Z}$ as

$$
\begin{aligned}
& \hat{Z}=e^{-\beta(\hat{H}-\mu \hat{N})}, \\
& Z=\operatorname{Tr}\{\hat{Z}\} .
\end{aligned}
$$

The Entropy operator [cf. Ref. [126] Eq. (2)] then can be written as

$$
\hat{S}=-\log \hat{\rho}=\beta(\epsilon-\mu) \hat{\Psi}^{\dagger} \hat{\Psi}+\log (Z)
$$

and

$$
-\hat{\rho} \log \hat{\rho}=\frac{\beta(\epsilon-\mu)}{Z} e^{-\beta(\epsilon-\mu) \hat{\Psi}^{\dagger} \hat{\Psi}} \hat{\Psi}^{\dagger} \hat{\Psi}+\frac{\log (Z)}{Z} e^{-\beta(\epsilon-\mu) \hat{\Psi}^{\dagger} \hat{\Psi}}
$$


The operator $\hat{Z}$ has the series expansion

$$
\begin{aligned}
\hat{Z} & =\exp \left\{\left(-\beta(\epsilon-\mu) \hat{\Psi}^{\dagger} \hat{\Psi}\right)\right\} \\
& =1-\beta(\epsilon-\mu) \hat{\Psi}^{\dagger} \hat{\Psi}+\frac{\beta^{2}(\epsilon-\mu)^{2}}{2 !} \hat{\Psi}^{\dagger} \hat{\Psi} \hat{\Psi}^{\dagger} \hat{\Psi}+\ldots \\
& =1+\hat{\Psi}^{\dagger} \hat{\Psi}\left(-1+1-\beta(\epsilon-\mu)+\frac{\beta^{2}(\epsilon-\mu)^{2}}{2 !}+\ldots\right) \\
& =1+\hat{\Psi}^{\dagger} \hat{\Psi}\left(e^{-\beta(\epsilon-\mu)}-1\right) .
\end{aligned}
$$

Here we have used the fact that $\left(\hat{\Psi}^{\dagger} \hat{\Psi}\right)^{2}=\hat{\Psi}^{\dagger} \hat{\Psi}$ for fermions. In the third line, we have added and subtracted 1 to obtain the exponential series. Note also that the first term in the last line is 1 and its action on state vectors is that of the identity operator on the many-body Hilbert space. Therefore, when we take the trace, the first term contributes twice (once for the vacuum and once for the single fermi level $\epsilon)$ :

$$
Z=\operatorname{Tr}\{\hat{Z}\}=1+1+\left(e^{-\beta(\epsilon-\mu)}-1\right)=1+e^{-\beta(\epsilon-\mu)} .
$$

The entropy can be calculated by taking the trace of Eq. (5.10)

$$
\begin{aligned}
S & =\frac{\beta(\epsilon-\mu) e^{-\beta(\epsilon-\mu)}}{1+e^{-\beta(\epsilon-\mu)}}+\log Z \\
& =\beta(\epsilon-\mu) f(\epsilon)+\log \left(1+e^{-\beta(\epsilon-\mu)}\right) \\
& =-(f \log f+(1-f) \log (1-f)),
\end{aligned}
$$

where a simple algebraic manipulation can show that line 2 and line 3 above are the same. The fermi function gives the occupancy of the single fermi level of energy $\epsilon$ :

$$
f(\epsilon)=\left\langle\hat{\Psi}^{\dagger} \hat{\Psi}\right\rangle=\frac{1}{1+e^{\beta(\epsilon-\mu)}} .
$$

The argument presented above for the entropy can be made even more obvious by explicitly constructing the density matrix in the Hilbert space of a single fermionic level. The Hilbert space has exactly two orthonormal vectors which we label as $\{|0\rangle,|1\rangle\}$ corresponding to the vacuum state and the single-particle state of energy $\epsilon$ respectively. It is easy to see that the density matrix given by Eq. (5.6) is diagonal in this basis and that

$$
\hat{\rho}=\left(\begin{array}{cc}
\langle 0|\hat{\rho}| 0\rangle & \langle 0|\hat{\rho}| 1\rangle \\
\langle 1|\hat{\rho}| 0\rangle & \langle 1|\hat{\rho}| 1\rangle
\end{array}\right)=\left(\begin{array}{cc}
1-f & 0 \\
0 & f
\end{array}\right)=\left(\begin{array}{cc}
\frac{1}{Z} & 0 \\
0 & \frac{e^{-\beta(\epsilon-\mu)}}{Z}
\end{array}\right) .
$$


Therefore the probability associated with the vacuum is $1-f$ while the probability associated with the level $\epsilon$ is $f$. The expressions for the entropy operator and entropy follow directly from the above expression. It is also straight-forward to calculate other average quantities.

In the second quantized notation, we may write Eq. (5.15) as

$$
\hat{\rho}=\left((1-f) \hat{\Psi} \hat{\Psi}^{\dagger}+f \hat{\Psi}^{\dagger} \hat{\Psi}\right) .
$$

\subsubsection{Generalization to Many States}

It is straight forward to generalize the above analysis to a (noninteracting) system with multiple levels. The Hamiltonian and number operators are then given by

$$
\begin{aligned}
& \hat{H}=\sum_{i} \hat{H}_{i}=\sum_{i} \epsilon_{i} \hat{\Psi}_{i}^{\dagger} \hat{\Psi}_{i} \\
& \hat{N}=\sum_{i} \hat{N}_{i}=\sum_{i} \hat{\Psi}_{i}^{\dagger} \hat{\Psi}_{i} .
\end{aligned}
$$

We note that the Hamiltonian and number operators corresponding to different levels always commute

$$
\left[\hat{N}_{i}, \hat{N}_{j}\right]=0 .
$$

Therefore the density operator can be written as

$$
\hat{\rho}=\frac{e^{-\beta(\hat{H}-\mu \hat{N})}}{\operatorname{Tr}\left\{e^{-\beta(\hat{H}-\mu \hat{N})}\right\}}=\frac{\prod_{i} e^{-\beta\left(\epsilon_{i}-\mu\right) \hat{\Psi}_{i}^{\dagger} \hat{\Psi}_{i}}}{Z},
$$

where

$$
\begin{aligned}
Z=\operatorname{Tr}\{\hat{Z}\} & =\operatorname{Tr}\left\{\prod_{i} e^{-\beta\left(\epsilon_{i}-\mu\right) \hat{\Psi}_{i}^{i} \hat{\Psi}_{i}}\right\} \\
& =\prod_{i}\left(1+e^{-\beta\left(\epsilon_{i}-\mu\right)}\right)
\end{aligned}
$$

where one has to use the fact that $\left[\hat{\Psi}_{i}, \hat{N}_{j}\right]=\delta_{i j} \Psi_{i}$. The expression for $Z$ above is merely the product of $Z$ over the different states $i$. This means that $\log Z$ will be additive over the different states.

The Entropy Operator $\hat{S}$ is

$$
\begin{aligned}
\hat{S}=-\log \hat{\rho} & =\log Z+\beta(\hat{H}-\mu \hat{N}) \\
& =\beta(\hat{H}-\mu \hat{N}-\Omega),
\end{aligned}
$$


where the grand canonical potential is defined as $\Omega=-\beta \log Z$. The expectation value of $\hat{S}$ is

$$
S=\langle\hat{S}\rangle=\frac{\beta}{Z} \operatorname{Tr}\left\{(\hat{H}-\mu \hat{N}) e^{-\beta(\hat{H}-\mu \hat{N})}\right\}+\log Z .
$$

This may be simplified by noting that

$$
\begin{aligned}
\frac{1}{Z} \operatorname{Tr}\left\{(\hat{H}-\mu \hat{N}) e^{-\beta(\hat{H}-\mu \hat{N})}\right\} & =-\frac{\partial \log Z}{\partial \beta} \\
& =-\frac{\partial}{\partial \beta}\left(\sum_{i} \log \left(1+e^{-\beta\left(\epsilon_{i}-\mu\right)}\right)\right) \\
& =\sum_{i} \frac{\left(\epsilon_{i}-\mu\right) e^{-\beta\left(\epsilon_{i}-\mu\right)}}{1+e^{-\beta\left(\epsilon_{i}-\mu\right)}} \\
& =\sum_{i}\left(\epsilon_{i}-\mu\right) f\left(\epsilon_{i}\right)
\end{aligned}
$$

which gives an analogous expression to Eq. (5.13) but with a sum over states

$$
\begin{aligned}
S & =\sum_{i}\left(\beta\left(\epsilon_{i}-\mu\right) f_{i}+\log \left(1+e^{-\beta\left(\epsilon_{i}-\mu\right)}\right)\right) \\
& =\sum_{i}-\left(f_{i} \log f_{i}+\left(1-f_{i}\right) \log \left(1-f_{i}\right)\right) .
\end{aligned}
$$

The above equation tells us that the entropy is additive for independent fermions which is what we would expect [128].

The above equation for entropy motivates us to write down the density matrix in a form given by Eq. (5.15):

$$
\hat{\rho}=\otimes_{k} \hat{\rho}_{k}=\otimes_{k}\left(\begin{array}{cc}
1-f_{k} & 0 \\
0 & f_{k}
\end{array}\right) .
$$

The above density operator represents $N$ uncorrelated systems where $N$ corresponds to the number of energy levels available to a single particle. Each energy level, along with the corresponding vacuum, behaves as an independent system. Each of these Hilbert spaces have two state vectors $\left\{|0\rangle_{n},|1\rangle_{n}\right\}$ corresponding to the vacuum and the one particle level of energy $\epsilon_{n}$. The entire Hilbert space is then a direct product of such Hilbert spaces and its density operator will have the form given in Eq. (5.25) when these systems are uncorrelated. 
In the second quantized notation, we write

$$
\hat{\rho}=\prod_{k}\left(\left(1-f_{k}\right) \hat{\Psi}_{k} \hat{\Psi}_{k}^{\dagger}+f_{k} \hat{\Psi}_{k}^{\dagger} \hat{\Psi}_{k}\right) .
$$

The joint entropy theorem [129] tells us that the entropy of uncorrelated systems has to be additive

$$
S(\hat{\rho})=\sum_{k} S\left(\hat{\rho}_{k}\right)=\sum_{k}-\left(f_{k} \log f_{k}+\left(1-f_{k}\right) \log \left(1-f_{k}\right)\right) .
$$

\subsection{Entropy in the Scattering Basis}

We now discuss the formulation of entropy in the context of a scattering problem. The system is composed of $M$ reservoirs which exchange particles and energy with each other through a central scattering region. Naturally, this is a nonequilibrium situation where there is a flux of particles and energy entering the reservoirs and thereby moving it away from equilibrium. However, the reservoirs are taken to be in the thermodynamic limit where it is assumed that their equilibrium configuration does not change. In this limit, the reservoirs set up the boundary conditions for the scattering problem.

The most natural framework to calculate quantities in the scattering problem are the scattering basis states. The scattering basis states associated with a reservoir are an orthogonal set of basis states. Furthermore, they are also orthogonal with respect to any other reservoir. We label the reservoirs $\alpha=\{1,2, \ldots, M\}$ and one has

$$
\begin{aligned}
\hat{H} & =\oplus_{\alpha} \hat{H}_{\alpha} \\
\hat{N} & =\oplus_{\alpha} \hat{N}_{\alpha},
\end{aligned}
$$

and the commutation relations

$$
\left[\hat{H}_{\alpha}, \hat{H}_{\beta}\right]=\left[\hat{N}_{\alpha}, \hat{N}_{\beta}\right]=0 .
$$

We label the reservoirs (corresponding states) as $\alpha$ (state $k$ ) and $\beta$ (state $k^{\prime}$ ), and write the anti-commutation relations

$$
\left\{\hat{\Psi}_{\alpha, k}, \hat{\Psi}_{\beta, k^{\prime}}^{\dagger}\right\}=\delta_{\alpha \beta} \delta_{k k^{\prime}}
$$


The density operator in the scattering basis is simply an extension of the density operator given by Eq. (5.26) with an additional product over scattering states corresponding to different reservoirs

$$
\prod_{k} \rightarrow \prod_{\alpha} \prod_{k_{\alpha}}
$$

Explicitly,

$$
\hat{\rho}=\prod_{\alpha} \prod_{k_{\alpha}}\left(\left(1-f_{k_{\alpha}}\right) \hat{\Psi}_{k_{\alpha}} \hat{\Psi}_{k_{\alpha}}^{\dagger}+f_{k_{\alpha}} \hat{\Psi}_{k_{\alpha}}^{\dagger} \hat{\Psi}_{k_{\alpha}}\right) .
$$

The density matrices are multiplicative and thereby entropies additive due to the commutation of the different subsystems [cf. Eq. (5.29)] and we directly use Eq. (5.21) to write the entropy operator as

$$
\hat{S}=\sum_{\alpha} \hat{S}_{\alpha}=\sum_{\alpha} \beta_{\alpha}\left(\hat{H}_{\alpha}-\mu_{\alpha} \hat{N}_{\alpha}-\Omega_{\alpha}\right)
$$

In the same operator representation, we may even write the differential form of the first law as

$$
\delta \hat{S}=\sum_{\alpha} \delta \hat{S}_{\alpha}=\sum_{\alpha} \beta_{\alpha}\left(\delta \hat{H}_{\alpha}-\mu_{\alpha} \delta \hat{N}_{\alpha}\right)
$$

The scattering states form a continuum and the entropy operator $\hat{S}_{\alpha}$ may be written as an integral over energy by introducing the density of states $g_{k_{\alpha}}(\omega)=$ $\sum_{k_{\alpha}} \delta\left(\omega-\epsilon_{k_{\alpha}}\right):$

$$
\begin{aligned}
\hat{S}_{\alpha} & =\sum_{k}\left(\left(\epsilon_{k}-\mu_{\alpha}\right) \hat{\Psi}_{\alpha, k}^{\dagger} \hat{\Psi}_{\alpha, k}+\log \left(1+e^{-\beta_{\alpha}\left(\epsilon_{k}-\mu_{\alpha}\right)}\right)\right) \\
& =\int_{-\infty}^{\infty} g_{\alpha}(\omega)\left(\left(\omega-\mu_{\alpha}\right) \hat{\Psi}_{\alpha}^{\dagger}(\omega) \hat{\Psi}_{\alpha}(\omega)+\log \left(1+e^{-\beta_{\alpha}\left(\omega-\mu_{\alpha}\right)}\right)\right) .
\end{aligned}
$$

The corresponding entropy then is the expectation value of the above operator

$$
S_{\alpha}=-\sum_{k_{\alpha}}\left(f_{k_{\alpha}} \log f_{k_{\alpha}}+\left(1-f_{k_{\alpha}}\right) \log \left(1-f_{k_{\alpha}}\right)\right)
$$

and it is easy to see that

$$
S_{\alpha}=-\int_{-\infty}^{\infty} d \omega g_{\alpha}(\omega)\left(f_{\alpha}(\omega) \log f_{\alpha}(\omega)+\left(1-f_{\alpha}(\omega)\right) \log \left(1-f_{\alpha}(\omega)\right)\right),
$$


where $f_{\alpha} \equiv f_{\alpha}(\omega)$ is the Fermi-Dirac distribution associated with reservoir $\alpha$. The total entropy is simply the sum of the different reservoir contributions

$$
S=\sum_{\alpha} S_{\alpha}
$$

That is, in the absence of many-body interactions, the scattering states corresponding to different reservoirs do not mix and behave as uncorrelated systems.

The density of states $g_{\alpha}$ associated with reservoir $\alpha$ can be expressed in terms of the Green's function of the system

$$
g_{\alpha}(\omega)=\frac{1}{2 \pi} \operatorname{Tr}\left\{G^{r}(\omega) \Gamma^{\alpha}(\omega) G^{a}(\omega)\right\}
$$

where $\Gamma^{\alpha}$ is the tunneling-width matrix of the system with reservoir $\alpha$. Note that $\operatorname{Tr}\{$.$\} here refers to the trace over the one-body Hilbert space of the system. The$ Green's functions are in fact correlation functions which are calculated in the manybody Hilbert space. In reading the above equation, the Green's functions are understood to be matrices (they are not operators in quantum-mechanical sense) acting on the one-body Hilbert space of the system. We refer the reader to the book by Stefanucci and van Leeuwan [39] for details pertaining to the Green's function formalism and appendix A for relevant details pertaining to the nonequilibrium steady state. Eq. (5.39) gives us the partial density of states [31, 130] of the system associated with reservoir $\alpha$. The total density of states is given by

$$
\begin{aligned}
g(\omega)=\sum_{\alpha} g_{\alpha}(\omega) & =\sum_{\alpha} \frac{1}{2 \pi} \operatorname{Tr}\left\{G^{r}(\omega) \Gamma^{\alpha}(\omega) G^{a}(\omega)\right\} \\
& =\sum_{\alpha} \operatorname{Tr}\left\{A_{\alpha}(\omega)\right\} \\
& =\operatorname{Tr}\{A(\omega)\},
\end{aligned}
$$

where $A_{\alpha}(\omega)$ is the partial spectral function associated with reservoir $\alpha$ whereas $A(\omega)$ is the total spectral function.

\subsubsection{Entropy Matrix of the System}

The Green's functions and the spectral functions are matrices in the one-body Hilbert space of the system $\mathcal{H}_{1}$ which take into account exactly the open nature 
of the system via the tunneling-width matrices $\Gamma^{\alpha}$ which are also matrices $\mathrm{S}^{\dagger}$ in $\mathcal{H}_{1}$.

We may then write the entropy matrix $\mathbf{S}$ as

$$
\mathbf{S}=-\int_{-\infty}^{\infty} d \omega \sum_{\alpha} A_{\alpha}\left(f_{\alpha} \log f_{\alpha}+\left(1-f_{\alpha}\right) \log \left(1-f_{\alpha}\right)\right)
$$

where $A_{\alpha}$ is the matrix of the partial spectral function introduced in Eq. (5.40). Explicitly:

$$
A_{\alpha}(\omega)=\frac{1}{2 \pi} G^{r}(\omega) \Gamma^{\alpha}(\omega) G^{a}(\omega) .
$$

We suppressed the energy dependence of $A_{\alpha}$ and $f_{\alpha}$ in Eq. (5.41) for notational simplicity. The total spectral function was introduced in Eq. (5.40) and, for clarity, we write it explicitly:

$$
\begin{aligned}
A(\omega)=\sum_{\alpha} A_{\alpha}(\omega) & =\frac{1}{2 \pi} \sum_{\alpha} G^{r}(\omega) \Gamma^{\alpha}(\omega) G^{a}(\omega) \\
& =\frac{1}{2 \pi i}\left(G^{r}(\omega)-G^{a}(\omega)\right) .
\end{aligned}
$$

The matrices $A_{\alpha}$ and $\Gamma^{\alpha}$ can be shown to be positive-semidefinite [31, 41, 126]. This implies that the entropy matrix $\mathbf{S}$ is also positive-semidefinite. $A(\omega)=\sum_{\alpha} A_{\alpha}(\omega)$ integrates to

$$
\int_{-\infty}^{\infty} d \omega A(\omega)=\mathbb{1}
$$

the identity matrix in the one-body Hilbert space $\mathcal{H}_{1}$. The lesser Green's function provides information regarding the nonequilibrium occupancy of the system and can be written as

$$
G^{<}(\omega) \equiv G^{r}(\omega) \Sigma^{<}(\omega) G^{a}(\omega)=2 \pi i \sum_{\alpha} A_{\alpha}(\omega) f_{\alpha}(\omega)
$$

\subsubsection{Entropy of Subspaces}

The entropy matrix $\mathbf{S}$ given by Eq. 5.41) can be projected onto any subspace $\mathcal{A}$ of the one-body Hilbert space $\mathcal{H}_{1}$. We denote the entropy associated with the subspace $\mathcal{A}$ as $S[\mathcal{A}]$

$$
S[\mathcal{A}] \equiv \operatorname{Tr}\left\{P_{\mathcal{A}} \mathbf{S}\right\}
$$

\footnotetext{
${ }^{\dagger}$ As mentioned previously, we use the term matrices to highlight the fact that they are not operators in the Fock space but act on the one-body Hilbert space.
} 
where $P_{\mathcal{A}}$ is the projection operator for the subspace $\mathcal{A}$. We denote the partial density of states associated with the reservoir $\alpha$ and the subspace $\mathcal{A}$ as $g_{\mathcal{A}, \alpha} \equiv$ $\operatorname{Tr}\left\{P_{\mathcal{A}} A_{\alpha}\right\}$ so that

$$
S[\mathcal{A}]=\int_{-\infty}^{\infty} d \omega \sum_{\alpha} g_{\mathcal{A}, \alpha} \sigma\left(f_{\alpha}\right),
$$

where we introduce the function

$$
\sigma(f)=-(f \log f+(1-f) \log (1-f))
$$

and have suppressed the energy dependence of $g_{\mathcal{A}, \alpha}(\omega)$ and $f_{\alpha}(\omega)$ above for notational simplicity.

The subspace entropy has the important property that it is additive over orthogonal subspaces. The entropy associated with the subspace $\mathcal{C}=\mathcal{A} \oplus \mathcal{B}$ is clearly seen to be

$$
S[\mathcal{A} \oplus \mathcal{B}]=S[\mathcal{A}]+S[\mathcal{B}]
$$

since $P_{\mathcal{C}}=P_{\mathcal{A}}+P_{\mathcal{B}}$ when $P_{\mathcal{A}} P_{\mathcal{B}}=0$ for the orthogonal subspaces $\mathcal{A}, \mathcal{B}$. The total entropy then can be easily written as a sum of entropies over orthogonal subspaces of the one-body Hilbert space $\mathcal{H}_{1}$.

We may therefore introduce an entropy density $S(\mathbf{x})$ using the projection operator in the position basis $P(\mathbf{x})=|\mathbf{x}\rangle\langle\mathbf{x}|$

$$
S(\mathbf{x})=\int_{-\infty}^{\infty} d \omega \sum_{\alpha} g_{\alpha}(\mathbf{x}) \sigma\left(f_{\alpha}\right)
$$

where

$$
g_{\alpha}(\mathbf{x})=\operatorname{Tr}\left\{P(\mathbf{x}) A_{\alpha}\right\}=\left\langle\mathbf{x}\left|A_{\alpha}\right| \mathbf{x}\right\rangle
$$

and since the projection operator $P(\mathbf{x})$ obey the completeness relation

$$
\int d^{3} \mathbf{x}|\mathbf{x}\rangle\langle\mathbf{x}|=\mathbb{1}
$$

we find that the total entropy of the system is

$$
S=\int d^{3} \mathbf{x} S(\mathbf{x})
$$

The total entropy above is completely equivalent to the one given by Eq. (5.38). 


\subsection{Entropy Inferred from Local Measurements}

Local measurements do not have access to information regarding the scattering states of the system. Consider for example a one dimensional transport problem where a quantum wire is connected to two reservoirs: $\mathrm{L}$ (on the left) and R (on the right). A local observer cannot distinguish between states that are moving left (these are scattering states associated with $\mathrm{R}$ ) and ones that are moving right (scattering states associated with $\mathrm{L}$ ). This lack of information leads to a mixing of the states corresponding to $\mathrm{L}$ and $\mathrm{R}$. We show that this lack of information implies an increase in the observed entropy.

The local observer sees a nonequilibrium distribution which is related to the local projection of $G^{<}$on the subspace $\mathcal{A}$ of the one-body Hilbert space $\mathcal{H}_{1}$ which she has access to. She also observes a local spectrum which she has access to:

$$
\begin{aligned}
g_{\mathcal{A}}(\omega) & =\operatorname{Tr}\left\{P_{\mathcal{A}} A(\omega)\right\} \\
& =\sum_{\alpha} \operatorname{Tr}\left\{P_{\mathcal{A}} A_{\alpha}(\omega)\right\} \\
& \equiv \sum_{\alpha} g_{\mathcal{A}, \alpha} .
\end{aligned}
$$

The nonequilibrium distribution function sampled by the local observer in the subspace $\mathcal{A}$ is

$$
f_{\mathcal{A}} \equiv \frac{\operatorname{Tr}\left\{P_{\mathcal{A}} G^{<}(\omega)\right\}}{2 \pi i g_{\mathcal{A}}(\omega)}
$$

Using Eq. (5.45) for $G^{<}$, we find that

$$
f_{\mathcal{A}}=\frac{\sum_{\alpha} g_{\mathcal{A}, \alpha} f_{\alpha}}{g_{\mathcal{A}}}
$$

The quantities $g_{\mathcal{A}}$ and $f_{\mathcal{A}}$ above are clearly measurable by scanning probe techniques and near-field photoemission. Experiments generally provide position-local measurements where the projection operator can be written as $P(\mathbf{x})=|\mathbf{x}\rangle\langle\mathbf{x}|$ but covering a finite region of space

$$
P_{\mathcal{A}} \equiv \int_{\mathbf{x} \in \mathcal{A}} d^{3} \mathbf{x} P(\mathbf{x})
$$


The nonequilibrium distribution function has been discussed in Refs. [31, 41, 131] and has also been covered in Chap. 2 of the present thesis.

The observer sees the system to be locally out of equilibrium and would believe the system to have the density matrix given by

$$
\hat{\rho}=\otimes_{n} \hat{\rho}_{n}=\otimes_{n}\left(\begin{array}{cc}
1-f_{\mathcal{A}}\left(\epsilon_{n}\right) & 0 \\
0 & f_{\mathcal{A}}\left(\epsilon_{n}\right)
\end{array}\right) .
$$

The index $n$ runs over the local spectrum of states seen by the observer $g_{\mathcal{A}}(\omega)=$ $\sum_{n} \delta\left(\omega-\epsilon_{n}\right)$. The entropy can then be written as

$$
S_{s}[\mathcal{A}]=-\int_{-\infty}^{\infty} d \omega g_{\mathcal{A}}(\omega)\left(f_{\mathcal{A}}(\omega) \ln f_{\mathcal{A}}(\omega)+\left(1-f_{\mathcal{A}}(\omega)\right) \ln \left(1-f_{\mathcal{A}}(\omega)\right)\right) .
$$

We write the above entropy with the subscript "s" to differentiate it from the entropy calculated with full knowledge of the scattering basis. Our prior work utilized the same subscript for the nonequilibrium distribution function $f_{s}$ as well. Although we designated the nonequilibrium distribution function in Eq. 5.55 with the subscript " $\mathcal{A}$ ", we used "s" to designate the same function sampled locally by a probe. The reader may refer to the subscript "s" as "system locally."

$$
S_{s}[\mathcal{A}]=\int_{-\infty}^{\infty} d \omega g_{\mathcal{A}}(\omega) \sigma\left(f_{\mathcal{A}}\right)
$$

The mean particle number and energies of the subsystem $\mathcal{A}$ are then

$$
\begin{aligned}
& N_{\mathcal{A}}=\int_{-\infty}^{\infty} d \omega g_{\mathcal{A}}(\omega) f_{\mathcal{A}}(\omega) \\
& E_{\mathcal{A}}=\int_{-\infty}^{\infty} \omega d \omega g_{\mathcal{A}}(\omega) f_{\mathcal{A}}(\omega) .
\end{aligned}
$$

We note that choosing a completely local measurement with $P_{\mathcal{A}}=P(\mathbf{x})$ gives us the entropy density $S_{s}(\mathbf{x})=S_{s}[\mathcal{A}]$, the particle density $N(\mathbf{x})=N_{\mathcal{A}}$ and the energy density $E(\mathbf{x})=E_{\mathcal{A}}$. We then designate the local density of states by $g(\omega ; \mathbf{x})=g_{\mathcal{A}}(\omega)$ and the local nonequilibrium distribution as $f(\omega ; \mathbf{x})=f_{\mathcal{A}}(\omega)$.

\subsection{Entropy Inferred from a Probe Measurement}

In chapter 2 we covered in great detail the notion of a probe measurement. We noted that the probe measurement of temperature and voltage is simply the following 
problem: Finding an equilibrium Fermi-Dirac distribution $f_{p}(\omega)$ which has the same energy and occupancy of the nonequilibrium system it probes. The probe chooses a subspace $\mathcal{A}$ and the voltage and temperature measurement finds the Fermi-Dirac distribution $f\left(\mu_{p}, T_{p} ; \omega\right)=f_{p}(\omega)$ such that

$$
\begin{aligned}
& N_{\mathcal{A}}=\left.\int_{-\infty}^{\infty} d \omega g_{\mathcal{A}}(\omega) f_{p}(\omega) \equiv N_{\mathcal{A}}\right|_{f_{p}} \\
& E_{\mathcal{A}}=\left.\int_{-\infty}^{\infty} d \omega \omega g_{\mathcal{A}}(\omega) f_{p}(\omega) \equiv E_{\mathcal{A}}\right|_{f_{p}},
\end{aligned}
$$

where the particle number $N_{\mathcal{A}}$ and energy $E_{\mathcal{A}}$ of the subsystem, appearing in the lhs above, were defined by Eq. (5.61). Eq. (5.62) are the constraints that define the probe temperature and chemical potential which was discussed in chapter 2 in its most general form.

We showed that Eq. (5.62) uniquely determines $f_{p}(\omega) \equiv f\left(\mu_{p}, T_{p} ; \omega\right)$ if and only if both the constraints are imposed. We interpreted this as a consequence of the second law of thermodynamics. The density matrix for the probe distribution may be constructed in exactly the same way as was done in Eq. (5.58):

$$
\hat{\rho}_{p}=\otimes_{n} \hat{\rho}_{n}=\otimes_{n}\left(\begin{array}{cc}
1-f_{p}\left(\epsilon_{n}\right) & 0 \\
0 & f_{p}\left(\epsilon_{n}\right)
\end{array}\right),
$$

where the spectrum $g_{\mathcal{A}}(\omega)=\sum_{n} \delta\left(\omega-\epsilon_{n}\right)$ with energies $\epsilon_{n}$ in the subspace $\mathcal{A}$ are now filled with an equilibrium distribution function $f_{p}$. We may then write the entropy associated with the equilibrium distribution function $f_{p}$ in the same way as Eq. (5.60):

$$
\begin{aligned}
S_{p}[\mathcal{A}] & =-\int_{-\infty}^{\infty} d \omega g_{\mathcal{A}}(\omega)\left(f_{p}(\omega) \ln f_{p}(\omega)+\left(1-f_{p}(\omega)\right) \ln \left(1-f_{p}(\omega)\right)\right) \\
& =\int_{-\infty}^{\infty} d \omega g_{\mathcal{A}}(\omega) \sigma\left(f_{p}\right) .
\end{aligned}
$$

Once again, if we talk about purely local measurements $P_{\mathcal{A}}=P(\mathbf{x})$, we shall designate the entropy density measured by the probe as $S_{p}(\mathbf{x})$. We would of course expect the equilibrium entropy $S_{p}$ to be larger than the corresponding nonequilibrium $S_{s}$ subject to the same constraints [Eq. (5.62)]. We now discuss the inequalities satisfied by the entropies $S, S_{s}$ and $S_{p}$. 


\subsection{Entropy Inequalities}

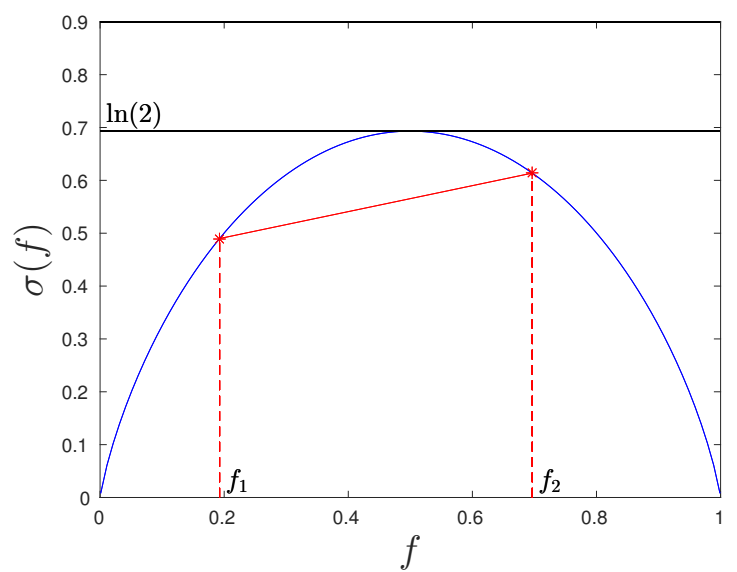

Figure 5.1: The function $\sigma(f)=-f \ln f-(1-f) \ln (1-f)$. Illustration of its convexity: $\sigma\left(\lambda f_{1}+(1-\lambda) f_{2}\right) \geq \lambda \sigma\left(f_{1}\right)+(1-\lambda) \sigma\left(f_{2}\right)$.

We note that the function $\sigma$ is convex and thereby obeys the Jensen's inequality [132]:

$$
\sigma\left(\lambda f_{1}+(1-\lambda) f_{2}\right) \geq \lambda \sigma\left(f_{1}\right)+(1-\lambda) \sigma\left(f_{2}\right)
$$

as illustrated in Fig. 5.1. When $\lambda \in[0,1], f=\lambda f_{1}+(1-\lambda) f_{2}$ is a point on the $\mathrm{x}-$ axis between $f_{1}$ and $f_{2}$ whose function value $\sigma(f)$ is greater than the corresponding point on the line segment shown in red $\lambda \sigma\left(f_{1}\right)+(1-\lambda) \sigma\left(f_{2}\right)$ which connects points $P_{1}=\left(f_{1}, \sigma\left(f_{1}\right)\right)$ and $P_{2}=\left(f_{2}, \sigma\left(f_{2}\right)\right)$. In other words, the blue curve is always above the red line between points $P_{1}$ and $P_{2}$. The equality holds when $\lambda=0$ or 1 , or when $f_{1}=f_{2}$.

We may generalize the inequality for $M$ points

$$
\sigma\left(\sum_{i} \lambda_{i} f_{i}\right) \geq \sum_{i} \lambda_{i} \sigma\left(f_{i}\right)
$$

where $\sum_{i} \lambda_{i}=1$ and $\lambda_{i} \geq 0 \forall i \in\{1,2,3 \ldots M\}$. We apply this directly to entropy 
$S_{s}[\mathcal{A}]$ in Eq. 5.60

$$
\begin{aligned}
S_{s}[\mathcal{A}] & =\int_{-\infty}^{\infty} d \omega g_{\mathcal{A}} \sigma\left(f_{\mathcal{A}}\right) \\
& =\int_{-\infty}^{\infty} d \omega g_{\mathcal{A}} \sigma\left(\frac{\sum_{\alpha} g_{\mathcal{A}, \alpha} f_{\alpha}}{g_{\mathcal{A}}}\right) \\
& \geq \int_{-\infty}^{\infty} d \omega \sum_{\alpha} g_{\mathcal{A}, \alpha} \sigma\left(f_{\alpha}\right) \equiv S[\mathcal{A}]
\end{aligned}
$$

where we used the fact that $g_{\mathcal{A}}=\sum_{\alpha} g_{\mathcal{A}, \alpha}$ from Eq. (5.54) and also the fact that $g_{\mathcal{A}} f_{\mathcal{A}}=\sum_{\alpha} g_{\mathcal{A}, \alpha} f_{\alpha}$ as shown in Eq. 5.56 .

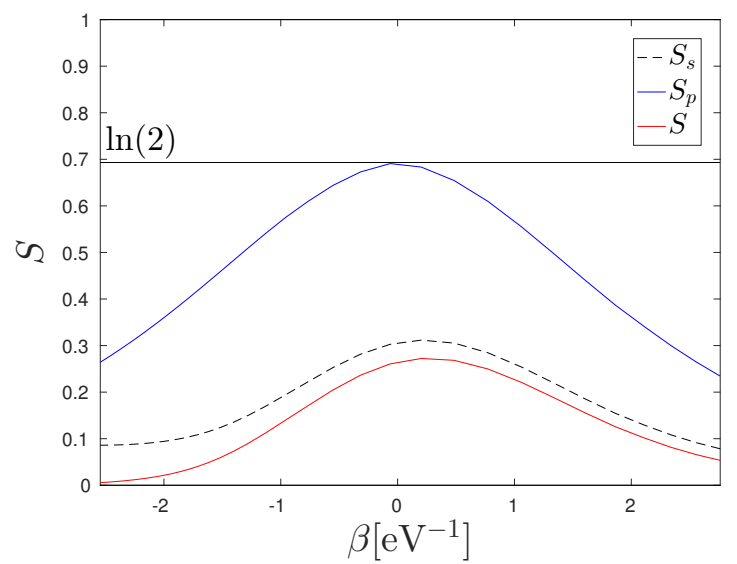

Figure 5.2: Subsystem entropy $S[\mathcal{A}]$ of a two-level quantum system far from equilibrium, plotted versus the inverse temperature $\beta=1 / T_{p}$ of the probe. The electrical bias across the system was varied from 1.6 to $3.2 \mathrm{~V}$. Values of $\beta<0$ correspond to absolute negative temperatures (population inversion). We see that $S[\mathcal{A}] \leq S_{s}[\mathcal{A}] \leq S_{p}[\mathcal{A}]$

Consider the entropy $S_{s}[\mathcal{A}]$ but supposing we do not have access to the nonequilibrium distribution function $f_{\mathcal{A}}$ but do have method to measure the mean particle number $N_{\mathcal{A}}$ and the mean energy $E_{\mathcal{A}}$ associated with the subsystem $\mathcal{A}$. We see that the equilibrium distribution $f=f_{p}$ extremizes the entropy subject to the constraits of fixed particle number and energy in the following way. We write the variations 
in the entropy subject to these constraints as

$$
\begin{aligned}
\delta S[f]= & \delta\left(\int_{-\infty}^{\infty} d \omega g_{\mathcal{A}}(\omega) \sigma(f)\right. \\
& \left.+\lambda_{1}\left[\int_{-\infty}^{\infty} d \omega g_{\mathcal{A}}(\omega) f(\omega)-N_{\mathcal{A}}\right]+\lambda_{2}\left[\int_{-\infty}^{\infty} d \omega \omega g_{\mathcal{A}}(\omega) f(\omega)-E_{\mathcal{A}}\right]\right)
\end{aligned}
$$

where $\lambda_{1}, \lambda_{2}$ are the Lagrange multipliers associated with the constraints of fixed particle number $N_{\mathcal{A}}$ and fixed energy $E_{\mathcal{A}}$ respectively. $N_{\mathcal{A}}$ and $E_{\mathcal{A}}$ were defined in Eq. (5.61) and the constraint was explicitly stated in the section 5.4. We find that

$$
\delta S[f]=\int_{-\infty}^{\infty} d \omega g_{\mathcal{A}}(\omega)\left[-\log \left(\frac{f}{1-f}\right)+\lambda_{1}+\omega \lambda_{2}\right] \delta f
$$

and extremizing it $(\delta S=0)$ for any variation $\delta f$ would imply that the integrand above vanishes and we obtain

$$
f=\frac{1}{1+e^{-\lambda_{1}-\omega \lambda_{2}}} .
$$

The usual identification of these multipliers is

$$
\lambda_{1}=\frac{\mu_{p}}{T_{p}}, \lambda_{2}=-\frac{1}{T_{p}} .
$$

It was shown in Chap. 2 that the Fermi-Dirac distribution which has the same particle number and energy is a unique one. We see here that the same Fermi-Dirac distribution extremizes the entropy subject to those constraints. Furthermore, we know that the entropy

$$
S[f]=\int_{-\infty}^{\infty} d \omega g_{\mathcal{A}}(\omega) \sigma(f)
$$

is convex. We can explicitly write the second order variation of the entropy

$$
\delta^{2} S[f]=\int_{-\infty}^{\infty} d \omega g_{\mathcal{A}}(\omega)\left[-\frac{1}{f}-\frac{1}{1-f}\right] \delta f^{2},
$$

which is always negative since $0 \leq f \leq 1$. Therefore, $f_{p}$ in fact maximizes the entropy subject to the constraints and we have

$$
S[\mathcal{A}] \leq S_{s}[\mathcal{A}] \leq S_{p}[\mathcal{A}]
$$




\begin{tabular}{|c|c|c|}
\hline Notation & Meaning & $\begin{array}{c}\text { Information Available } \\
\text { (from the measurement) }\end{array}$ \\
\hline $\mathbf{S}$ & $\begin{array}{c}\text { Entropy Matrix } \\
\left.\text { ( in } \mathcal{H}_{1}\right)\end{array}$ & $\begin{array}{c}\text { Complete Information } \\
\text { of the Scattering States }\end{array}$ \\
\hline$S[\mathcal{A}]$ & $\begin{array}{c}\text { Projection of } \mathbf{S} \\
\text { in subspace } \mathcal{A}\end{array}$ & $\begin{array}{c}\text { Complete Information } \\
\text { of the Scattering States }\end{array}$ \\
\hline$S_{s}[\mathcal{A}]$ & $\begin{array}{c}\text { subscript s for "system locally" } \\
\text { in subspace } \mathcal{A}\end{array}$ & $\begin{array}{c}\text { Local Distribution Function } \\
\text { Local Density of States }\end{array}$ \\
\hline$S_{p}[\mathcal{A}]$ & subscript p for "probe" & $\begin{array}{c}\text { Distribution Unknown } \\
\text { Local DOS Known }\end{array}$ \\
\hline
\end{tabular}

Table 5.1: Table summarizing the notation and meaning of the different entropies based on local measurements. The entropies are inversely related to the information that is available to the local observer. Equilibrium probe entropy $S_{p}[\mathcal{A}]$ is the greatest of the three entropies and the observer in this case has the least amount of information regarding the system (colored in red).

The equality holds only when all the reservoirs are maintained at the same temperature and chemical potential, i.e., in equilibrium. The inequality is illustrated for a two level system out of equilibrium in Fig. 5.2. Table 5.1 summarizes the relationship between the observed entropy and the information available to the observer.

We therefore find that full knowledge of the scattering states of the system leads to the least entropy $S[\mathcal{A}]$ for any subspace $\mathcal{A}$. Indeed, this entropy vanishes when all the reservoirs are maintained at zero temperature, consistent with the third law of thermodynamics. However, the system may still appear to be out of equilibrium for a local observer. If the observer has access only to the local distribution but no knowledge of the scattering states, it would appear to the observer that the distribution does not correspond to the one at zero temperature. A local observer with access only to the local distribution always sees an increased entropy. Furthermore, if the observer has no idea about the local distribution but only knows the temperature and voltage by performing local probe measurements, the entropy increases even further. We therefore see the intimate relationship between the access to information pertaining to the system and the observed entropy. 


\subsection{Entropy as a Metric for Local Equilibrium Departure}

We posit that the local entropy difference can be used a metric quantifying local equilibrium departure. Fig. 5.2 shows that the entropy of the auxiliary equilibrium system $S_{p}[\mathcal{A}]$ follows the local entropy $S_{s}[\mathcal{A}]$ as well as the entropy of the scattering states $S[\mathcal{A}]$. The probe entropy $S_{p}[\mathcal{A}]$ reaches a maximum value at infinite temperature which also corresponds, more or less, to the maximum of $S_{s}[\mathcal{A}]$ as measured by the local observer without knowledge of the scattering states as well as the maximum value of entropy $S[\mathcal{A}]$ evaluated with complete knowledge of the scattering states. Local experiments are capable of measuring $S_{p}[\mathcal{A}]$ and $S_{s}[\mathcal{A}]$ of a subspace $\mathcal{A}$. $\mathcal{A}$ is usually a position-local subspace: Scanning tunneling microscopy can be used to measure the local temperature and chemical potential and thereby calculate $S_{p}[\mathcal{A}]$; Photo-emission measurements can be used to measure the local nonequilibrium distribution $f_{s}$ and thereby calculate the associated entropy $S_{s}[\mathcal{A}]$. The entropy difference

$$
\Delta S=S_{p}-S_{s} \geq 0
$$

is a metric which quantifies the departure from equilibrium. Clearly, when all reservoirs are maintained at the same temperature and chemical potential, i.e., at equilibrium,

$$
\Delta S=0
$$

Scanning tunneling measurements can be used to measure the local density of states. In this context, we stated previously [cf. Eq. (3.4)] that the density of states seen by the probe is

$$
\bar{A}(\omega) \equiv \frac{\operatorname{Tr}\left\{\Gamma^{p}(\omega) A(\omega)\right\}}{\operatorname{Tr}\left\{\Gamma^{p}(\omega)\right\}},
$$

and the local nonequilibrium distribution seen by the probe [cf. Eq. (2.3)] is

$$
f_{s}(\omega) \equiv \frac{\operatorname{Tr}\left\{\Gamma^{p}(\omega) G^{<}(\omega)\right\}}{2 \pi i \operatorname{Tr}\left\{\Gamma^{p}(\omega) A(\omega)\right\}},
$$

which can be measured using photo-emission experiments. It is shown in appendix A that $0 \leq f_{s} \leq 1$. We note that the above expressions are completely valid in the presence of arbitrary interactions within the quantum system. 
The expressions for the entropy seen by the local observer who has the knowledge of the local nonequilibrium distribution is then given by

$$
S_{s} \equiv S\left[f_{s}(\omega)\right]=-\int_{-\infty}^{\infty} d \omega \bar{A}(\omega)\left[f_{s}(\omega) \ln f_{s}(\omega)+\left(1-f_{s}(\omega)\right) \ln \left(1-f_{s}(\omega)\right)\right],
$$

and the entropy inferred from a probe measurement is

$$
S_{p} \equiv S\left[f_{p}(\omega)\right]=-\int_{-\infty}^{\infty} d \omega \bar{A}(\omega)\left[f_{p}(\omega) \ln f_{p}(\omega)+\left(1-f_{p}(\omega)\right) \ln \left(1-f_{p}(\omega)\right)\right] .
$$

Following the arguments of the previous section, it can be shown that

$$
S_{p} \leq S_{s}
$$

even in the presence of interactions. Again, the equality holds only at equilibrium.

For sufficiently low probe temperatures,

$$
S_{s} \leq S_{p} \simeq \frac{\pi^{2}}{3} \bar{A}\left(\mu_{0}\right) k_{B} T_{p}
$$

the leading order term in the Sommerfeld expansion of Eq. (5.78). It is easy to see that the local entropy $S_{s} \leq S_{p} \rightarrow 0$ as the probe temperature approaches absolute zero $T_{p} \rightarrow 0$, consistent with the third law of thermodynamics.

It is important to note that Eqs. (5.77) and (5.78) are valid for arbitrary interactions within the quantum system. However, their theoretical basis is not at the same foundational level as that of their corresponding entropies in Eqs. (5.60) and (5.64) respectively, which were derived for the noninteracting case. The latter expressions for the entropy have a rigorous derivation in terms of the system's scattering states but the presence of interactions causes the scattering states to mix and therefore makes their generalization considerably harder. We therefore note that the entropy [Eq. (5.77)] given in this section is a working ansatz whenever interactions are present.

\subsubsection{Per-state Entropy Deficit}

We argued that the local entropy deficit $\Delta S=S_{p}-S_{s}$ is a suitable metric quantifying the departure from equilibrium. However, it is important to note that the mean 
local spectrum $\bar{A}(\omega)$ varies significantly from point to point within the nanostructure depending upon the local probe-system coupling (especially in the tunneling regime) and limits the use of $\Delta S$ while comparing the 'distance' from equilibrium for points within the nanostructure. The situation is analogous to that of a dilute gas, which can have a very low entropy per unit volume even if it has a very high entropy per particle. We note that states far from the equilibrium Fermi energy $\mu_{p}$ contribute negligibly to the entropy since $\lim _{f \rightarrow 0} S[f]=\lim _{f \rightarrow 1} S[f]=0$, and therefore introduce a normalization averaged over the thermal window of the probe:

$$
\mathcal{N}=\int_{-\infty}^{\infty} d \omega \frac{\bar{A}(\omega)}{\operatorname{Tr}\{A(\omega)\}}\left(\frac{-\partial f_{p}}{\partial \omega}\right)
$$

We define the local entropy-per-state of the system $s_{s}$ and that of the corresponding local equilibrium distribution $s_{p}$ as

$$
\begin{aligned}
& s_{s}=\frac{S_{s}}{\mathcal{N}}, \\
& s_{p}=\frac{S_{p}}{\mathcal{N}} .
\end{aligned}
$$

$\Delta s=s_{p}-s_{s}$ quantifies the per-state 'distance' from local equilibrium. We present numerical calculations of the local entropy-per-state below and discuss its implications.

\subsubsection{Numerical Results}

The local temperature distributions shown in Figs. 3.1 3.3 (presented in chapter 3) are essentially outside the scope of linear response theory [63] since the cold reservoir $R 1$ is held at $T_{1}=0 \mathrm{~K}$, and derivatives of the Fermi function are singular at $T=0 \mathrm{~K}$. However, it is an open question how far out of equilibrium these systems are and which regions therein manifest the most fundamentally nonequilibrium character. To address such questions quantitatively, we use the concept of local entropy per state introduced here. In particular, the normalized local entropy deficit $\Delta s \equiv s_{p}-s_{s}$ allows us to quantify how far the system is from local equilibrium.

Fig. 5.3 shows the local entropy distribution of the system $s_{s}$ and that of the corresponding local equilibrium distribution $s_{p}$, defined by Eqs. (5.82) and (5.83), 

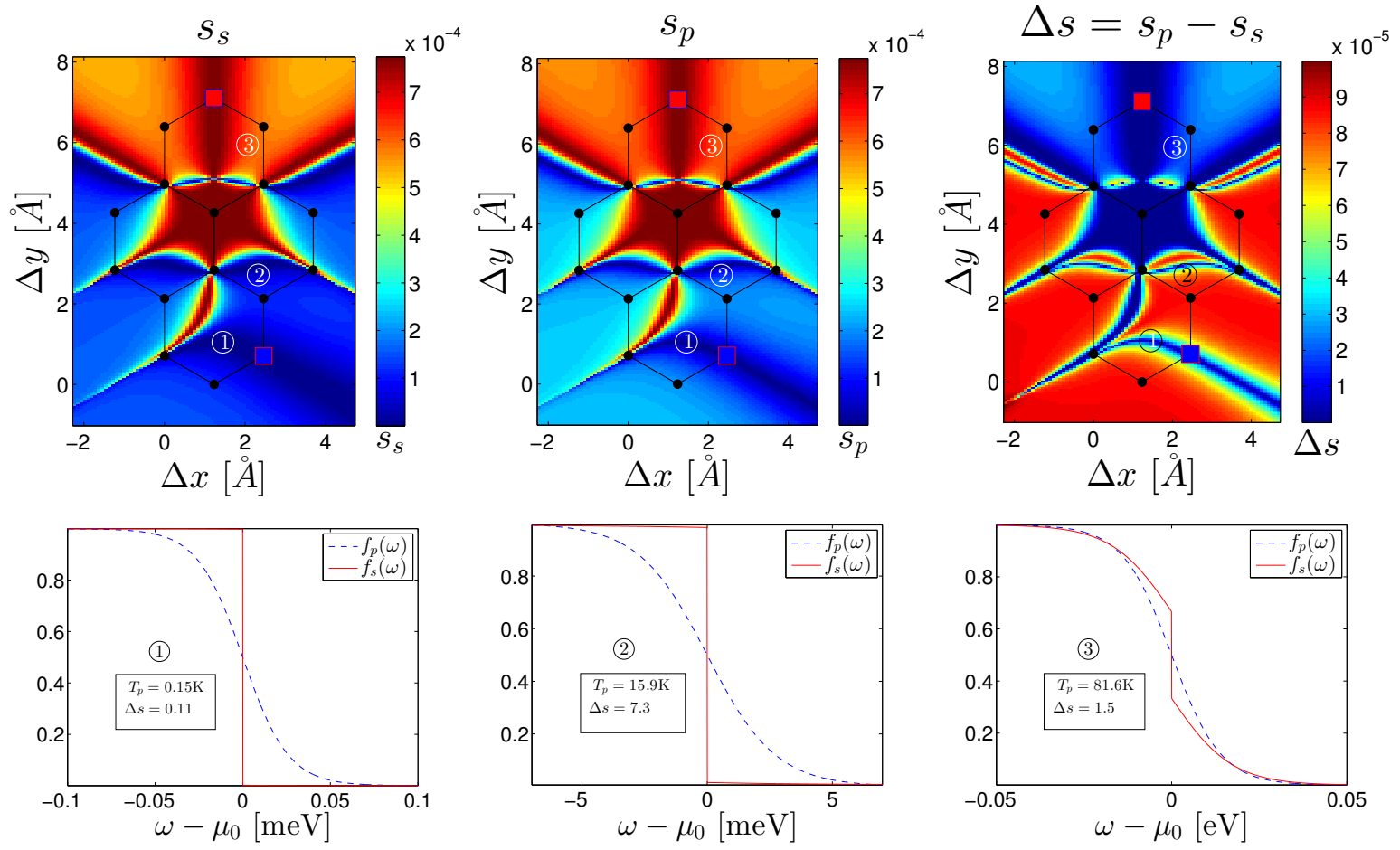

Figure 5.3: Upper panels (left to right): The local entropy-per-state of the system $s_{s}$, of the corresponding local equilibrium distribution $s_{p}$, and the local entropy deficit $\Delta s \equiv s_{p}-s_{s}$. The temperature distribution for the same junction (with identical bias conditions and sampling of probe positions) is shown in Fig. 3.2, and we note that it resembles almost exactly the distribution $s_{p}$. Lower panels: The distributions $f_{s}$ and $f_{p}$ for three points shown in the upper panels, each having different probe temperatures $T_{p}=0.15 \mathrm{~K}, 15.9 \mathrm{~K}$ and $81.6 \mathrm{~K}$, respectively. The corresponding entropy deficits are $\Delta s=0.11,7.3$, and 1.5 , respectively, $\times 10^{-5}$. Point 2, although closer to $0 \mathrm{~K}$ than point 3 is to $100 \mathrm{~K}$, is further from local equilibrium.

respectively, for the Au-pyrene-Au junction considered in chapter 3, Fig. 3.2. The $s_{p}$ distribution strongly resembles the temperature distribution shown in Fig. 3.2 , consistent with the fact [cf. Eq. (5.80)] that the equilibrium entropy of a system of fermions is proportional to temperature at low temperatures. This resemblence is only manifest in the properly-normalized entropy per state $s_{p}$; the spatial variations of $S_{p}$ are much larger, and stem from the orders-of-magnitude variations of the local density of states $\bar{A}\left(\mu_{0}\right)$. The nonequilibrium entropy distribution $s_{s}$ of the system qualitatively resembles $s_{p}$, but everywhere satisfies the inequality $s_{s} \leq s_{p} . s_{s} \rightarrow 0$ whenever $T_{p} \rightarrow 0$, consistent with the third law of thermodynamics. 
The deviation from local equilibrium quantified by the local entropy deficit $\Delta s=s_{p}-s_{s}$ is shown in the top right panel of Fig. 5.3. $\Delta s$ shows deep blue regions (low entropy deficit) in both the hottest and coldest parts of the system, while the largest entropy deficits (bright red) occur in the areas at intermediate temperatures. This may be explained as follows: within elastic transport theory, the local nonequilibrium distribution function is a linear combination of the distribution functions of the various reservoirs (refer to appendix Con elastic transport). The entropy deficit is minimal when this distribution function strongly resembles the equilibrium Fermi-Dirac distribution of one of these reservoirs. Conversely, the entropy deficit is maximal when there is a large admixture of both hot and cold electrons without inelastic processes leading to equilibration. Therefore, the hottest and coldest spots show the smallest entropy deficits since there is very little mixing from the cold reservoir $R 1$ and hot reservoir $R 2$, respectively, while the regions at intermediate temperatures have the largest entropy deficitis, and hence are farthest from local equilibrium.

However, it can be seen that the colder spots are more strongly affected due to the mixing from the hot reservoir $R 2$, while the hotter spots are affected to a lesser extent due to the mixing from the cold reservoir $R 1$. This reflects the fact that the distribution function $f_{s}$ deviates much more from the distribution function $f_{1}(\omega)$ with $T_{1} \rightarrow 0$ (implying a pure state with zero entropy) due to a small admixture of hot electrons from $R 2$ than is the case for the opposite scenario. In other words, it is easier to increase the entropy deficit $\Delta s$ of a cold spot by adding hot electons (and thus driving it out of equilibrium) than it is the other way round. The entropy deficit is a good metric to capture such a change in the distribution function and gives us a per-state "distance" from local equilibrium.

\subsection{Conclusions}

We systematically developed a notion for the nonequilibrium entropy in terms of the scattering states for a system of noninteracting fermions in a steady-state. We then 
formulated the entropy that is measured by a local observer who has no knowledge whatsoever of the scattering states but has detailed knowledge of the local spectrum and local distribution function. We find that this lack of knowledge increases the observed entropy. Finally, we formulated the entropy that is measured without knowledge of the local distribution function but knowledge of the local mean particle number and energy. We show that the entropies formulated in the three different ways satisfy a hierarchy of inequalities with the most knowledgable formulation leading to the least entropy. We illustrated our results for a two-level system far from equilibrium. We propose an ansatz for the entropy when two-body interactions are present. We show that the ansatz is consitent with the second and third laws of thermodynamics. Based on the inequalities developed, we also use the entropy as a metric to quantify the "distance" from equilibrium. We illustrate the latter point using numerical calculations on a molecular junction driven far from equilibrium. 


\title{
CHAPTER 6
}

\author{
Concluding Remarks
}

In the present thesis, we presented a number of interesting results pertaining to steady-state quantum fermion systems. In chapter 2 we defined a mathematically rigorous notion of temperature and voltage for quantum systems arbitrarily far from equilibrium and having arbitrary interactions within the system. We showed that a meaningful notion of temperature and voltage for nonequilibrium systems requires the simultaneous measurement of both. This joint measurement requires that the probe be in both electrical and thermal equilibrium with the nonequilibrium system of interest. We established the notion of an ideal probe as one that operates in the broadband limit and is weakly coupled to the system of interest. The results obtained here have a deep connection with the second law of thermodynamics: We proved the positive-definiteness of the Onsager matrix of response coefficients and interpreted it as a statement of the second law of thermodynamics which ensures the uniqueness of the probe measurement. We derived necessary and sufficient conditions for the existence of a solution and found that a solution always exists, and that one may encounter negative temperature solutions if the system is driven sufficienty far away from equilibrium. We developed also the notion of entropy for steady-state noninteracting systems and found a hierarchy of inequalities which have an intimate relationship with the information that is available to an observer making local measurements. We also proposed a novel experimental method, whose working principle rests our theoretical findings, to enhance the existing spatial resolution of scanning thermal measurements by over two orders of magnitude. In addition to their theoretical merit we believe these results will have significant practical impact, for example, in analyzing nonequilibrium device performances.

The work presented here leaves plenty of interesting open problems. For example, we did not consider the case where the fermionic spin degeneracy is broken 
such as magnetic systems. The first law of thermodynamics would then have an additional magnetic term and it is unclear whether the additional thermodynamic variable (magnetic moment) entering the first law can be defined uniquely outside equilibrium. Furthermore, we did not consider thermodynamic measurements of the bosonic degrees of freedom which is an interesting question to pursue. Notions of nonequilibrium entropy were presented rigorously in the absence of interactions and, though we provide an ansatz in the presence of interactions, it appears to be a formidable theoretical challenge when two-body interactions are present. We also did not comment upon the relationship of our notion of entropy to the entanglement entropy. It is also noteworthy that almost all of the analysis in this area pertains to a nonequilibrium steady-state. It is entirely unclear how one might conceive of these thermodynamic notions in the realm of time-varying nonequilibrium states.

From a broader perspective, nonequilibrium thermodynamics is a rapidly developing field of study and it is certainly a very exciting time for theoretical work. Open quantum systems have been studied from a dynamical viewpoint [133] (where system + environment undergoes unitary evolution but the system dynamics described by the reduced density matrix is nonunitary), e.g. using Master equation approaches, and the notion of decoherence has had considerable success in explaining the quantum to classical transition. The emergence of thermodynamic equilibrium states from this underlying process of decoherence has been dubbed quantum thermodynamics and has seen a flurry of activity in recent years [134]. There are also approaches to nonequilibrium thermodynamics which take ideas from quantum information theory where thermal nonequilibrium states are seen as "resources" [135]; for example, such resources may be consumed as fuel to achieve an erasure operation which irreversibly generates entropy. There is also a great impetus on the experimental side, for example, a recent experiment [136] employed two initially quantum-correlated spins (a resource) to reverse the thermodynamic arrow of time, i.e., flow of heat from the colder system to the hotter one; this of course is not a violation of the Clausius statement since the heat flow is not the "sole effect". Stochastic thermodynamics [137] is a relatively recent development which aims to 
extend notions of classical irreversible thermodynamics to the level of individual particle trajectories and is now being increasingly employed to study nonequilibrium quantum systems, e.g., in the context of the irreversibility brought about by a quantum measurement [138]. In summary, there is a great ongoing effort to understand some of the most fundamental aspects of quantum mechanics and thermodynamics. The confluence of these many different approaches, including the Green's function approach, to nonequilibrium thermodynamics of quantum systems is sure to create many fruitful discussions in the years to come. 


\section{APPENDIX A}

The Nonequilibrium Steady State

We consider a system whose Hamiltonian $\hat{H}$ is independent of time, but is driven out of equilibrium, e.g., by electrical and/or thermal bias. The nonequilibrium steady state is described by a density matrix $\hat{\rho}$ that is time-independent. The expectation values of observables are given by their usual prescription in statistical physics

$$
\langle\hat{Q}\rangle=\operatorname{Tr}\{\hat{\rho} \hat{Q}\}=\sum_{\mu, \nu} \rho_{\mu \nu}\langle\nu|\hat{Q}| \mu\rangle
$$

The "lesser" and "greater" Green's functions [39] used in the thesis are defined as follows

$$
G_{\alpha \beta}^{<}(t) \equiv i\left\langle d_{\beta}^{\dagger}(0) d_{\alpha}(t)\right\rangle
$$

while its Hermitian conjugate is

$$
G_{\alpha \beta}^{>}(t) \equiv-i\left\langle d_{\alpha}(t) d_{\beta}^{\dagger}(0)\right\rangle
$$

where

$$
d_{\alpha}(t)=e^{i \frac{\hat{H}}{\hbar} t} d_{\alpha}(0) e^{-i \frac{\hat{H}}{\hbar} t}
$$

evolves according to the Heisenberg equation of motion for a system with Hamiltonian $\hat{H}$. Here, $\alpha, \beta$ denote basis states in the 1-body Hilbert space of the system.

The spectral representation uses the eigenbasis of the Hamiltonian $\hat{H}|\nu\rangle=E_{\nu}|\nu\rangle$, where $\nu$ denotes a many-body energy eigenstate. One may write the "lesser" Green's function as

$$
\begin{aligned}
G_{\alpha \beta}^{<}(\omega)=2 \pi i \sum_{\mu, \mu^{\prime}, \nu} \rho_{\mu \nu}\left\langle\nu\left|d_{\beta}^{\dagger}\right| \mu^{\prime}\right\rangle\left\langle\mu^{\prime}\left|d_{\alpha}\right| \mu\right\rangle \\
\times \delta\left(\omega-\frac{E_{\mu}-E_{\mu^{\prime}}}{\hbar}\right),
\end{aligned}
$$


while the "greater" Green's function becomes

$$
\begin{aligned}
G_{\alpha \beta}^{>}(\omega)=-2 \pi i \sum_{\mu, \mu^{\prime}, \nu} \rho_{\mu \nu} & \left\langle\nu\left|d_{\alpha}\right| \mu^{\prime}\right\rangle\left\langle\mu^{\prime}\left|d_{\beta}^{\dagger}\right| \mu\right\rangle \\
& \times \delta\left(\omega-\frac{E_{\mu^{\prime}}-E_{\nu}}{\hbar}\right) .
\end{aligned}
$$

The spectral function $A(\omega)$ is given by

$$
A(\omega) \equiv \frac{1}{2 \pi i}\left(G^{<}(\omega)-G^{>}(\omega)\right),
$$

and can be expressed in the spectral representation as

$$
\begin{array}{r}
A_{\alpha \beta}(\omega)=\sum_{\mu, \mu^{\prime}, \nu}\left[\rho_{\mu \nu}\left\langle\nu\left|d_{\beta}^{\dagger}\right| \mu^{\prime}\right\rangle\left\langle\mu^{\prime}\left|d_{\alpha}\right| \mu\right\rangle\right. \\
\left.+\rho_{\nu \mu^{\prime}}\left\langle\mu^{\prime}\left|d_{\alpha}\right| \mu\right\rangle\left\langle\mu\left|d_{\beta}^{\dagger}\right| \nu\right\rangle\right] \\
\quad \times \delta\left(\omega-\frac{E_{\mu}-E_{\mu^{\prime}}}{\hbar}\right) .
\end{array}
$$

\section{A.0.1 Sum Rule for the Spectral Function}

Eq. A.8 leads to the following sum rule for the spectral function:

$$
\begin{aligned}
\int_{-\infty}^{\infty} d \omega A_{\alpha \beta}(\omega) & =\sum_{\mu, \nu} \rho_{\mu \nu}\left\langle\nu\left|d_{\beta}^{\dagger} d_{\alpha}\right| \mu\right\rangle \\
& +\sum_{\mu^{\prime}, \nu} \rho_{\nu \mu^{\prime}}\left\langle\mu^{\prime}\left|d_{\alpha} d_{\beta}^{\dagger}\right| \nu\right\rangle \\
& =\sum_{\mu, \nu} \rho_{\mu \nu}\left\langle\nu\left|d_{\beta}^{\dagger} d_{\alpha}+d_{\alpha} d_{\beta}^{\dagger}\right| \mu\right\rangle \\
& =\sum_{\mu, \nu} \rho_{\mu \nu}\left\langle\nu\left|\delta_{\alpha \beta}\right| \mu\right\rangle \\
& =\sum_{\mu, \nu} \rho_{\mu \nu} \delta_{\mu \nu} \delta_{\alpha \beta} \\
& =\delta_{\alpha \beta} \operatorname{Tr}\{\hat{\rho}\} \\
& =\delta_{\alpha \beta} .
\end{aligned}
$$

In our theory of local thermodynamic measurements, the quantity of interest is the local spectrum of the system sampled by the probe $\bar{A}(\omega)$, defined in Eq. 2.55). This obeys a further sum rule in the broadband limit (ideal probe), discussed below. 


\section{Local Spectrum in the Broadband Limit}

The probe-system coupling is energy independent in the broadband limit, $\Gamma^{p}(\omega)=$ const, and we write $\operatorname{Tr}\left\{\Gamma^{p}\right\}=\bar{\Gamma}^{p}$ for its trace. The local spectrum sampled by the probe $\bar{A}(\omega)$ defined in Eq. 2.55 can be written in the broadband limit as

$$
\bar{A}(\omega)=\frac{1}{\bar{\Gamma}^{p}} \sum_{\alpha, \beta}\left\langle\beta\left|\Gamma^{p}\right| \alpha\right\rangle A_{\alpha \beta}(\omega) .
$$

In this limit, it obeys a further sum rule:

$$
\begin{aligned}
\int_{-\infty}^{\infty} d \omega \bar{A}(\omega) & =\frac{1}{\bar{\Gamma}^{p}} \sum_{\alpha, \beta}\left\langle\beta\left|\Gamma^{p}\right| \alpha\right\rangle \int_{-\infty}^{\infty} d \omega A_{\alpha \beta}(\omega) \\
& =\frac{1}{\bar{\Gamma}^{p}} \sum_{\alpha, \beta}\left\langle\beta\left|\Gamma^{p}\right| \alpha\right\rangle \delta_{\alpha \beta} \\
& =1 .
\end{aligned}
$$

The broadband limit is special in that the measurement is determined by the local properties of the system itself, and is not influenced by the spectrum of the probe. In this limit, the local spectrum $\bar{A}(\omega)$ obeys the sum rule A.11 since the probe samples the same subsystem at all energies. One should not expect such a local sum rule to hold outside the broadband limit, since the probe samples different subsystems at different energies.

\section{A.0.2 Diagonality of $\hat{\rho}$}

We have, for any observable $\hat{Q}$,

$$
\begin{aligned}
\langle\hat{Q}(t)\rangle & =\sum_{\mu, \nu} \rho_{\mu \nu}\langle\nu|\hat{Q}(t)| \mu\rangle \\
& =\sum_{\mu, \nu} \rho_{\mu \nu}\left\langle\nu\left|e^{i \frac{\hat{H}}{\hbar} t} \hat{Q} e^{-i \frac{\hat{H}}{\hbar} t}\right| \mu\right\rangle \\
& =\sum_{\mu, \nu} \rho_{\mu \nu} e^{-i \frac{E_{\mu}-E_{\nu}}{\hbar} t}\langle\nu|\hat{Q}| \mu\rangle .
\end{aligned}
$$

The system observables must be independent of time in steady state. Therefore $\hat{\rho}$ must be diagonal in the energy basis, as seen from the above equation. The 
nondiagonal parts of $\hat{\rho}$ in the energy basis, when they exist, must be in a degenerate subspace so that $E_{\mu}=E_{\nu}$ in the above equation.

For states degenerate in energy, the boundary conditions determining the nonequilibrium steady state will determine the basis in which $\hat{\rho}$ is diagonal. Henceforth, we work in that basis.

\section{A.0.3 Positivity of $-i G^{<}(\omega)$ and $i G^{>}(\omega)$}

Working in the energy eigenbasis in which $\hat{\rho}$ is diagonal,

$$
\begin{aligned}
& -i\left\langle\alpha\left|G^{<}(\omega)\right| \alpha\right\rangle \equiv-i G_{\alpha \alpha}^{<}(\omega)= \\
& 2 \pi \sum_{\mu, \mu^{\prime}} \rho_{\mu \mu}\left|\left\langle\mu\left|d_{\alpha}^{\dagger}\right| \mu^{\prime}\right\rangle\right|^{2} \delta\left(\omega-\frac{E_{\mu}-E_{\mu^{\prime}}}{\hbar}\right) \geq 0 .
\end{aligned}
$$

Similarly,

$$
\begin{aligned}
& i\left\langle\alpha\left|G^{>}(\omega)\right| \alpha\right\rangle \equiv i G_{\alpha \alpha}^{>}(\omega)= \\
& 2 \pi \sum_{\mu, \mu^{\prime}} \rho_{\mu \mu}\left|\left\langle\mu\left|d_{\alpha}^{\dagger}\right| \mu^{\prime}\right\rangle\right|^{2} \delta\left(\omega-\frac{E_{\mu^{\prime}}-E_{\mu}}{\hbar}\right) \geq 0 .
\end{aligned}
$$

It follows that

$$
\langle\alpha|A(\omega)| \alpha\rangle=\frac{1}{2 \pi}\left\langle\alpha\left|-i G^{<}(\omega)+i G^{>}(\omega)\right| \alpha\right\rangle \geq 0
$$

Therefore, all three operators $-i G^{<}(\omega), i G^{>}(\omega)$, and $A(\omega)$ are positive-semidefinite.

\section{A. $0.40 \leq f_{s}(\omega) \leq 1$}

The nonequilibrium distribution function $f_{s}(\omega)$ was defined in Eq. (2.3) as

$$
f_{s}(\omega) \equiv \frac{\operatorname{Tr}\left\{\Gamma^{p}(\omega) G^{<}(\omega)\right\}}{2 \pi i \operatorname{Tr}\left\{\Gamma^{p}(\omega) A(\omega)\right\}}
$$

We have $\Gamma^{p}(\omega)>0$ by causality [39]:

$$
\operatorname{Im} \Sigma_{p}^{r}(\omega)=-\frac{1}{2} \Gamma^{p}(\omega)<0
$$


Let $\Gamma^{p}\left|\gamma_{p}\right\rangle=\gamma_{p}\left|\gamma_{p}\right\rangle$, where $\gamma_{p} \geq 0$ and some $\gamma_{p}$ satisfy $\gamma_{p}>0$. The energy dependence is taken to be implicit. The traces in Eq. A.16) may be evaluated in the eigenbasis of $\Gamma^{p}$, yielding:

$$
\begin{aligned}
f_{s}(\omega) & =\frac{\sum_{\gamma_{p}} \gamma_{p}\left\langle\gamma_{p}\left|G^{<}(\omega)\right| \gamma_{p}\right\rangle}{2 \pi i \sum_{\gamma_{p}} \gamma_{p}\left\langle\gamma_{p}|A(\omega)| \gamma_{p}\right\rangle} \\
& =\frac{\sum_{\gamma_{p}} \gamma_{p}\left\langle\gamma_{p}\left|-i G^{<}(\omega)\right| \gamma_{p}\right\rangle}{\sum_{\gamma_{p}} \gamma_{p}\left\langle\gamma_{p}\left|-i G^{<}(\omega)+i G^{>}(\omega)\right| \gamma_{p}\right\rangle}
\end{aligned}
$$

Therefore

$$
0 \leq f_{s}(\omega) \leq 1
$$




\section{APPENDIX B}

Noninvasive Probes

Our main results in chapter 2 relied upon the assumption of a noninvasive probe. We explained the physical basis for this assumption in Sec. 2.3.2, and we understood it to mean that the local probe-system transmission function $\mathcal{T}_{p s}$ and the local nonequilibrium distribution function $f_{s}$ are independent of the probe bias parameters $\left(\mu_{p}, T_{p}\right)$. In this appendix, we clarify the implicit mathematical details that have gone into this assumption of a noninvasive probe.

$f_{s}$ and $\mathcal{T}_{p s}$ have been defined in Eqs. 2.3 and (2.4), respectively, and they depend upon the Green's functions of the nonequilibrium quantum system. The Green's functions of the system do depend upon the probe parameters $\left(\mu_{p}, T_{p}\right)$ and we clarify this dependence. We label the probe parameter simply as $x_{p} \in\left\{\mu_{p}, T_{p}\right\}$, which can be taken to mean either the chemical potential or the temperature of the probe.

In order to characterize the noninvasive-probe limit, we introduce a dimensionless parameter $\lambda$, and write the probe-system coupling as $\Gamma^{p}(\omega)=\lambda \tilde{\Gamma}^{p}(\omega)$. Without

loss of generality, we may set $\operatorname{Tr}\left\{\tilde{\Gamma}^{p}\left(\mu_{0}\right)\right\}=\sum_{\alpha \neq p} \operatorname{Tr}\left\{\Gamma^{\alpha}\left(\mu_{0}\right)\right\}$, where $\Gamma^{\alpha}(\omega)$ is the tunneling-width matrix describing the coupling of lead $\alpha$ (e.g., source, drain, etc.) to the system, and $\mu_{0}$ is the equilibrium chemical potential of the system (or some other convenient reference value). The parameter

$$
\lambda \equiv \frac{\operatorname{Tr}\left\{\Gamma^{p}\left(\mu_{0}\right)\right\}}{\sum_{\alpha \neq p} \operatorname{Tr}\left\{\Gamma^{\alpha}\left(\mu_{0}\right)\right\}} \ll 1
$$

thus gives the condition for a weakly-coupled probe.

The currents flowing into the probe from the system are given by Eq. (2.2) as

$$
I_{p}^{(\nu)}=\frac{1}{h} \int_{-\infty}^{\infty} d \omega\left(\omega-\mu_{p}\right)^{\nu} \mathcal{T}_{p s}(\omega)\left[f_{s}(\omega)-f_{p}(\omega)\right]
$$


where

$$
\begin{aligned}
\mathcal{T}_{p s}= & \lambda 2 \pi \operatorname{Tr}\left\{\tilde{\Gamma}^{p} A\right\} \\
= & \lambda 2 \pi \operatorname{Tr}\left\{\left.\tilde{\Gamma}^{p} A\right|_{\lambda=0}\right\}+\lambda^{2} 2 \pi \operatorname{Tr}\left\{\left.\tilde{\Gamma}^{p} \frac{\partial A}{\partial \lambda}\right|_{\lambda=0}\right\} \\
& +\mathcal{O}\left(\lambda^{3}\right)
\end{aligned}
$$

and

$$
\begin{aligned}
\mathcal{T}_{p s} f_{s}= & -i \lambda \operatorname{Tr}\left\{\tilde{\Gamma}^{p} G^{<}\right\} \\
= & -i \lambda \operatorname{Tr}\left\{\left.\tilde{\Gamma}^{p} G^{<}\right|_{\lambda=0}\right\}-i \lambda^{2} \operatorname{Tr}\left\{\left.\tilde{\Gamma}^{p} \frac{\partial G^{<}}{\partial \lambda}\right|_{\lambda=0}\right\} \\
& +\mathcal{O}\left(\lambda^{3}\right)
\end{aligned}
$$

[cf. Eqs. 2.3 and 2.4)]. From Eqs. B.2 B.4), we see that $I_{p}^{(\nu)} \sim \mathcal{O}(\lambda)$. Similarly, it can be shown that

$$
\begin{aligned}
\frac{\partial I_{p}^{(\nu)}}{\partial x_{p}}= & -I_{p}^{(0)} \delta_{\nu, 1} \delta_{x_{p}, \mu_{p}} \\
& -\frac{\lambda}{\hbar} \int_{-\infty}^{\infty} d \omega\left(\omega-\mu_{p}\right)^{\nu} \operatorname{Tr}\left\{\left.\tilde{\Gamma}^{p} A\right|_{\lambda=0}\right\} \frac{\partial f_{p}}{\partial x_{p}} \\
& +\mathcal{O}\left(\lambda^{2}\right) .
\end{aligned}
$$

The leading-order results for the gradients are also $\mathcal{O}(\lambda)$, and agree with Eqs. 2.16) and (2.17). The noninvasive probe limit consists in keeping only the terms $\mathcal{O}(\lambda)$ in Eqs. (B.3), (B.4), and (B.5), and underlies the analysis presented in the body of the article. Deviations from the noninvasive probe limit appear as terms $\mathcal{O}\left(\lambda^{2}\right)$ and higher, which we now proceed to derive.

\section{B.0.1 Dependence of $G$ on $\lambda$ and $x_{p}$}

Standard NEGF arguments can be used to elucidate the dependence of the system Green's functions on $\lambda$ and $x_{p}$. Let $G_{0}$ denote the Green's function of the isolated quantum system without 2-body interactions, and let $\Sigma$ denote the self-energy describing 2-body interactions and coupling to various reservoirs, including the probe. 
Dyson's equation for the retarded (advanced) Green's function is [39]

$$
G^{r, a}=G_{0}^{r, a}+G_{0}^{r, a} \Sigma^{r, a} G^{r, a}
$$

The Keldysh equation for $G^{<}$is 39 .

$$
G^{<}(\omega)=G^{r}(\omega) \Sigma^{<}(\omega) G^{a}(\omega)
$$

where the "lesser" self-energy is

$$
\Sigma^{<}=i \lambda \tilde{\Gamma}^{p}(\omega) f_{p}(\omega)+i \sum_{\alpha \neq p} \Gamma^{\alpha}(\omega) f_{\alpha}(\omega)+\Sigma_{\text {int }}^{<}
$$

and $\Sigma_{\text {int }}^{<}$is the self-energy contribution due to electron-electron, electron-phonon, electron-photon interactions, etc. Similarly, the spectral function $A$ may be expressed as

$$
2 \pi A(\omega)=G^{r}(\omega) \Gamma(\omega) G^{a}(\omega)
$$

where

$$
\Gamma(\omega)=\lambda \tilde{\Gamma}^{p}(\omega)+\sum_{\alpha \neq p} \Gamma^{\alpha}(\omega)+\Gamma_{\mathrm{int}}(\omega)
$$

and $\Gamma_{\mathrm{int}}=i\left(\sum_{\mathrm{int}}^{r}-\Sigma_{\mathrm{int}}^{a}\right)$ is the contribution due to 2-body interactions. Note that all the terms appearing on the rhs of Eq. B.10 are positive definite due to causality.

Differentiating the self-energies with respect to $x_{p}$ gives

$$
\frac{\partial \Sigma^{<}}{\partial x_{p}}=i \lambda \tilde{\Gamma}^{p} \frac{\partial f_{p}}{\partial x_{p}}+\frac{\partial \Sigma_{\mathrm{int}}^{<}}{\partial x_{p}}
$$

and

$$
\frac{\partial \Sigma^{r, a}}{\partial x_{p}}=\frac{\partial \Sigma_{\mathrm{int}}^{r, a}}{\partial x_{p}} .
$$

Using Eqs. (B.6), (B.7), (B.11), and (B.12), it can be shown that

$$
\begin{gathered}
\frac{\partial G^{r, a}}{\partial x_{p}}=G^{r, a} \frac{\partial \Sigma_{\text {int }}^{r, a}}{\partial x_{p}} G^{r, a} \\
\frac{\partial G^{<}}{\partial x_{p}}=i \lambda G^{r} \tilde{\Gamma}^{p} G^{a} \frac{\partial f_{p}}{\partial x_{p}}+G^{r} \frac{\partial \Sigma_{\text {int }}^{<}}{\partial x_{p}} G^{a} \\
+G^{r} \frac{\partial \Sigma_{\text {int }}^{r}}{\partial x_{p}} G^{<}+G^{<} \frac{\partial \Sigma_{\text {int }}^{a}}{\partial x_{p}} G^{a} .
\end{gathered}
$$


Using $2 \pi i A=G^{a}-G^{r}$, the derivative of the spectral function may be written as

$$
2 \pi i \frac{\partial A(\omega)}{\partial x_{p}}=G^{a} \frac{\partial \Sigma_{\mathrm{int}}^{a}}{\partial x_{p}} G^{a}-G^{r} \frac{\partial \Sigma_{\mathrm{int}}^{r}}{\partial x_{p}} G^{r} .
$$

Finally, the derivatives of $\Sigma_{\text {int }}$ are given by

$$
\frac{\partial \Sigma_{\text {int }}^{\gamma}(\omega)}{\partial x_{p}}=\sum_{\eta=r, a,<} \int_{-\infty}^{\infty} d \omega^{\prime} K^{\gamma \eta}\left(\omega, \omega^{\prime}\right) \frac{\partial G^{\eta}\left(\omega^{\prime}\right)}{\partial x_{p}}
$$

where

$$
K^{\gamma \eta}\left(\omega, \omega^{\prime}\right) \equiv \frac{\delta \Sigma_{\text {int }}^{\gamma}(\omega)}{\delta G^{\eta}\left(\omega^{\prime}\right)}
$$

is the irreducible kernel for the 2-particle Green's function [39].

Eqs. (B.13), (B.14), and (B.16) are three coupled linear (integral) equations for $\partial G / \partial x_{p}$ and $\partial \Sigma_{\text {int }} / \partial x_{p}$. The only inhomogeneous term [first term on the rhs of Eq. B.14] is $\mathcal{O}(\lambda)$. Let

$$
\begin{gathered}
\frac{\partial G^{\gamma}(\omega)}{\partial x_{p}} \equiv \lambda F_{x_{p}}^{\gamma}(\omega), \\
\frac{\partial \Sigma_{\text {int }}^{\gamma}(\omega)}{\partial x_{p}} \equiv \lambda S_{x_{p}}^{\gamma}(\omega) .
\end{gathered}
$$

$F$ and $S$ satisfy the equations

$$
\begin{gathered}
F_{x_{p}}^{r, a}=G^{r, a} S_{x_{p}}^{r, a} G^{r, a} \\
F_{x_{p}}^{<}=i G^{r} \tilde{\Gamma}^{p} G^{a} \frac{\partial f_{p}}{\partial x_{p}}+G^{r} S_{x_{p}}^{<} G^{a} \\
+G^{r} S_{x_{p}}^{r} G^{<}+G^{<} S_{x_{p}}^{a} G^{a},
\end{gathered}
$$

and

$$
S_{x_{p}}^{\gamma}=\sum_{\eta=r, a,<} K^{\gamma \eta} F_{x_{p}}^{\eta}
$$

where the energy integral on the rhs of Eq. (B.22) is implicit. The leading-order solution is obtained by setting $G^{\gamma}=\left.G^{\gamma}\right|_{\lambda=0}$ in Eqs. B.20 and (B.21), so we see that $\partial G / \partial x_{p}, \partial \Sigma_{\text {int }} / \partial x_{p} \sim \mathcal{O}(\lambda)$, and can be neglected in the noninvasive probe limit. There exist a number of additional terms in $\partial G /\left.\partial \lambda\right|_{\lambda=0}$ that are independent of $x_{p}$, but these do not affect the proofs of theorems 1, 2, and 3 . 


\section{B.0.2 Proof of Uniqueness}

We are now in a position to evaluate the dependence of the currents $I_{p}^{(\nu)}$ on $x_{p} \in$ $\left\{\mu_{p}, T_{p}\right\}$. Taking the derivative of Eq. B.2 using the results of Appendix B.0.1. one obtains the exact expression

$$
\begin{aligned}
\frac{\partial I_{p}^{(\nu)}}{\partial x_{p}}= & -I_{p}^{(0)} \delta_{\nu, 1} \delta_{x_{p}, \mu_{p}}-\frac{\lambda}{h} \int_{-\infty}^{\infty} d \omega\left(\omega-\mu_{p}\right)^{\nu} \operatorname{Tr}\left\{\tilde{\Gamma}^{p} G^{r}\left(\Gamma-\Gamma^{p}\right) G^{a}\right\} \frac{\partial f_{p}}{\partial x_{p}} \\
& -\frac{i \lambda^{2}}{h} \int_{-\infty}^{\infty} d \omega\left(\omega-\mu_{p}\right)^{\nu} \operatorname{Tr}\left\{\tilde{\Gamma}^{p}\left(G^{r} S_{x_{p}}^{<} G^{a}+G^{r} S_{x_{p}}^{r} G^{<}+G^{<} S_{x_{p}}^{a} G^{a}\right)\right\} \\
& +\frac{i \lambda^{2}}{h} \int_{-\infty}^{\infty} d \omega\left(\omega-\mu_{p}\right)^{\nu} f_{p}(\omega) \operatorname{Tr}\left\{\tilde{\Gamma}^{p}\left(G^{a} S_{x_{p}}^{a} G^{a}-G^{r} S_{x_{p}}^{r} G^{r}\right)\right\} .
\end{aligned}
$$

To leading order in $\lambda$, Eq. (B.23) reduces to the result given in Eqs. (2.16) and (2.17), while the corrections are $\mathcal{O}\left(\lambda^{2}\right)$ or higher. Thus theorems 1 and 2 hold to leading order in $\lambda$ for systems with arbitary 2-body interactions, and the noninvasive-probe limit may be precisely defined as the limit $\lambda \ll 1$.

\section{Special Case: Noninteracting System}

Without 2-body interactions, only the first two terms in Eq. (B.23) survive. The current gradients therefore have the same form as in Eqs. 2.16) and (2.17), while

the $\mathcal{L}_{p s}^{(\nu)}$ coefficients have the same form [cf. Eq. 2.18] ] but with the transmission function replaced by

$$
\tilde{\mathcal{T}}_{p s}(\omega)=\operatorname{Tr}\left\{\Gamma^{p}(\omega) G^{r}(\omega)\left(\Gamma(\omega)-\Gamma^{p}(\omega)\right) G^{a}(\omega)\right\}
$$

which is positive due to causality [see Eq. (B.10)]. Theorem 1 therefore still holds. The uniqueness result as stated in theorem 2 holds also, since the argument only makes use of current gradients. We note that theorems 1 and 2 hold for arbitrarily strong probe couplings when 2-body interactions are absent. 


\section{Example: Hartree-Fock Approximation}

In the Hartree-Fock approximation, the irreducible kernel defined in Eq. (B.17) has the form

$$
K^{r<} \equiv \frac{\delta\left(\sum_{\mathrm{HF}}^{r}\right)_{n m}}{\delta\left(G^{<}\right)_{i j}}=U_{n j} \delta_{n m} \delta_{i j}-U_{n m} \delta_{n i} \delta_{m j},
$$

where $U_{n m}$ is the Coulomb integral between orthonormal basis orbitals $n$ and $m$ of the system. Furthermore, $K^{a<}=K^{r<}$ and $K^{<<}=K^{><}=0$. Eq. (B.22) therefore reduces to $S_{x_{p}}^{<}=0$ and

$$
\begin{aligned}
\left(S_{x_{p}}^{r, a}\right)_{n m}= & \delta_{n m} \sum_{j} U_{n j} \int_{-\infty}^{\infty} d \omega^{\prime}\left(F_{x_{p}}^{<}\left(\omega^{\prime}\right)\right)_{j j} \\
& -U_{n m} \int_{-\infty}^{\infty} d \omega^{\prime}\left(F_{x_{p}}^{<}\left(\omega^{\prime}\right)\right)_{n m} .
\end{aligned}
$$

\section{B.0.3 Proof of Existence}

The proof of Theorem 3 is based on an analysis of the quantities $\left.\langle\dot{N}\rangle\right|_{f_{s}},\left.\langle\dot{N}\rangle\right|_{f_{p}}$, $\left.\langle\dot{E}\rangle\right|_{f_{s}}$, and $\left.\langle\dot{E}\rangle\right|_{f_{p}}$ defined in Eqs. 2.38-2.41, respectively. These quantities are simply energy integrals of $\omega^{\nu} \mathcal{T}_{p s} f_{s}$ and $\omega^{\nu} \mathcal{T}_{p s} f_{p}$, with $\nu=0,1$, whose dependence on the small parameter $\lambda$ is given in Eqs. (B.3) and (B.4). Keeping only terms $\mathcal{O}(\lambda)$ (noninvasive-probe limit), these quantities reduce to the form considered in Sec. 2.5, so that Theorem 3 and Corollary 3.1 hold as before. Deviations from the noninvasive-probe limit involve corrections $\mathcal{O}\left(\lambda^{2}\right)$ and higher, and it is an open question whether a unique solution to the probe equilibration conditions 2.42 exists for arbitrarily strong probe-system coupling in the presence of interactions.

\section{Special Case: Noninteracting System}

For systems without 2-body interactions, the proof of Theorem 3 can be straightforwardly extended to the case of arbitrarily strong probe-system coupling. Using Eqs. (B.3) and (B.10) with $\Gamma_{\text {int }}=0$, one can write

$$
\mathcal{T}_{p s}=\sum_{\alpha} \operatorname{Tr}\left\{\Gamma^{p} G^{r} \Gamma^{\alpha} G^{a}\right\}
$$


Similarly, using Eqs. (B.4) and (B.8) with $\Sigma_{\text {int }}^{<}=0$, one has

$$
\mathcal{T}_{p s} f_{s}=\sum_{\alpha} \operatorname{Tr}\left\{\Gamma^{p} G^{r} \Gamma^{\alpha} G^{a}\right\} f_{\alpha}
$$

The probe equilibration conditions 2.42 whose solution we seek may be rewritten

$$
\begin{aligned}
\left.\langle\dot{N}\rangle\right|_{f_{s}}-\left.\langle\dot{N}\rangle\right|_{f_{p}} & =0 \\
\left.\langle\dot{E}\rangle\right|_{f_{s}}-\left.\langle\dot{E}\rangle\right|_{f_{p}} & =0
\end{aligned}
$$

The integrands in both conditions involve

$$
\begin{aligned}
\mathcal{T}_{p s}\left[f_{s}-f_{p}\right] & =\sum_{\alpha \neq p} \operatorname{Tr}\left\{\Gamma^{p} G^{r} \Gamma^{\alpha} G^{a}\right\}\left[f_{\alpha}-f_{p}\right] \\
& =\tilde{\mathcal{T}}_{p s}\left[\tilde{f}_{s}-f_{p}\right]
\end{aligned}
$$

where $\tilde{\mathcal{T}}_{p s}$ is given by Eq. B.24 and

$$
\tilde{f}_{s}=\frac{\sum_{\alpha \neq p} \operatorname{Tr}\left\{\Gamma^{p} G^{r} \Gamma^{\alpha} G^{a}\right\} f_{\alpha}}{\sum_{\alpha \neq p} \operatorname{Tr}\left\{\Gamma^{p} G^{r} \Gamma^{\alpha} G^{a}\right\}} .
$$

$\tilde{f}_{s}$ and $\tilde{\mathcal{T}}_{p s}$ are both independent of $x_{p}$ for the noninteracting system, and $\left.\langle\dot{N}\rangle\right|_{f_{s}}$, $\left.\langle\dot{N}\rangle\right|_{f_{p}},\left.\langle\dot{E}\rangle\right|_{f_{s}}$, and $\left.\langle\dot{E}\rangle\right|_{f_{p}}$ can be redefined using $\tilde{f}_{s}$ and $\tilde{\mathcal{T}}_{p s}$ without affecting the conditions B.29). Therefore the proofs of Theorem 3 and Corollary 3.1 hold for arbitrarily strong probe-system coupling in systems without 2-body interactions. 


\title{
APPENDIX C
}

\author{
Elastic Transport
}

We derive the form of the nonequilibrium distribution function $f_{s}(\omega)$ when the transport is dominated by elastic processes. We assume a nanostructure connected to $M$ reservoirs, including the probe. Eq. (2.2) takes the form of Eq. (3.13) when the transport is elastic, and we have

$$
\begin{aligned}
2 \pi \operatorname{Tr}\left\{\Gamma^{p}(\omega) A(\omega)\right\} & \left(f_{s}(\omega)-f_{p}(\omega)\right) \\
& =\sum_{\alpha=1}^{M} \mathcal{T}_{p \alpha}(\omega)\left(f_{\alpha}(\omega)-f_{p}(\omega)\right) .
\end{aligned}
$$

Now, we wish to rewrite the above equation in terms of the local properties sampled by the probe:

$$
\begin{aligned}
& \frac{\operatorname{Tr}\left\{\Gamma^{p}(\omega) A(\omega)\right\}}{\operatorname{Tr}\left\{\Gamma^{p}(\omega)\right\}}\left(f_{s}(\omega)-f_{p}(\omega)\right) \\
& =\sum_{\alpha=1}^{M} \frac{\operatorname{Tr}\left\{\Gamma^{p}(\omega) G^{r}(\omega) \Gamma^{\alpha}(\omega) G^{a}(\omega)\right\}}{2 \pi \operatorname{Tr}\left\{\Gamma^{p}(\omega)\right\}}\left(f_{\alpha}(\omega)-f_{p}(\omega)\right),
\end{aligned}
$$

where the first factor on the lhs is the mean local spectrum $\bar{A}(\omega)$ sampled by the probe, defined by Eq. (3.4), and we used Eq. (3.14 for the elastic transmissions on the rhs. We define the injectivity of a reservoir $\alpha$ sampled by the probe as

$$
\rho_{p \alpha}(\omega)=\frac{1}{2 \pi} \frac{\operatorname{Tr}\left\{\Gamma^{p}(\omega) G^{r}(\omega) \Gamma^{\alpha}(\omega) G^{a}(\omega)\right\}}{\operatorname{Tr}\left\{\Gamma^{p}(\omega)\right\}},
$$

for the factors appearing on the rhs of Eq. C.2. Injectivity of a reservoir $\alpha$ has been previously defined [130] as the local partial density of states (LPDOS) associated with the electrons originating from reservoir $\alpha$ and, due to number conservation, the sum of injectivities of the reservoirs gives the local density of states (LDOS). We state an equivalent result for the injectivities defined in Eq. (C.3) in the following paragraph. Before proceeding, we note that the injectivities sampled by the probe, in 
Eq. C.3), reduces to the LPDOS for electrons injected by reservoir $\alpha$ when the probe coupling is maximally local, i.e., $\left[\Gamma^{p}(\omega)\right]_{i j}=\Gamma^{p}(\omega) \delta_{i n} \delta_{j n}$ and becomes essentially independent of the probe coupling when it is weak. Eq. C.3 also extends to $\alpha=p$ and defines the probe injectivity sampled by itself, which becomes negligible in the limit of weak coupling.

It can be shown that the spectrum can be written as [9]

$$
A(\omega)=\frac{1}{2 \pi} G^{r}(\omega) \Gamma(\omega) G^{a}(\omega)
$$

where $\Gamma(\omega)$ is given by

$$
\Gamma(\omega)=\sum_{\alpha} \Gamma^{\alpha}(\omega)
$$

The contribution due to interactions $\Gamma^{\mathrm{int}}(\omega)$ in Eq. C.5 is missing since the interaction self-energy is Hermitian for elastic processes. Eqs. (C.3), C.4 and (C.5) imply:

$$
\sum_{\alpha=1}^{M} \rho_{p \alpha}(\omega)=\bar{A}(\omega)
$$

From Eq. C.2, we write

$$
\bar{A}(\omega)\left(f_{s}(\omega)-f_{p}(\omega)\right)=\sum_{\alpha=1}^{M} \rho_{p \alpha}(\omega)\left(f_{\alpha}(\omega)-f_{p}(\omega)\right)
$$

and Eq. C.6 implies

$$
\bar{A}(\omega) f_{s}(\omega)=\sum_{\alpha=1}^{M} \rho_{p \alpha}(\omega) f_{\alpha}(\omega)
$$

Finally, $f_{s}(\omega)$ can be written as

$$
\begin{aligned}
& f_{s}(\omega)=\sum_{\alpha=1}^{M} \frac{\rho_{p \alpha}(\omega)}{\bar{A}(\omega)} f_{\alpha}(\omega) . \\
& \therefore \quad 0 \leq f_{s}(\omega) \leq \sum_{\alpha=1}^{M} \frac{\rho_{p \alpha}(\omega)}{\bar{A}(\omega)} \\
& 0 \leq f_{s}(\omega) \leq 1
\end{aligned}
$$

where we used Eq. C.6 and the fact that the Fermi-Dirac distributions satisfy $0 \leq f_{\alpha}(\omega) \leq 1$. The nonequilibrium distribution function $f_{s}(\omega)$ is thus a linear combination of the Fermi-Dirac distributions of the reservoirs. 


\section{C.1 Linear Response Regime}

In cases where the transport occurs within the thermal energy window $k_{B} T_{0}$, the currents can be approximated to linear order in the bias

$$
I_{p}^{(\nu)}=\sum_{\alpha=1}^{M}\left[\mathcal{L}_{p \alpha}^{(\nu)}\left(\mu_{p}-\mu_{\alpha}\right)+\frac{\mathcal{L}_{p \alpha}^{(\nu+1)}}{T_{0}}\left(T_{p}-T_{\alpha}\right)\right]
$$

where the linear response coefficents are given by

$$
\begin{aligned}
\mathcal{L}_{p \alpha}^{(\nu)} & \equiv \mathcal{L}_{p \alpha}^{(\nu)}\left(\mu_{0}, T_{0}\right) \\
& =\frac{1}{h} \int_{-\infty}^{\infty} d \omega\left(\omega-\mu_{0}\right)^{\nu} \mathcal{T}_{p \alpha}(\omega)\left(-\frac{\partial f_{0}}{\partial \omega}\right),
\end{aligned}
$$

are evaluated at the mean chemical potential $\mu_{0}$ and mean temperature $T_{0}$ and $f_{0}$ is the Fermi-Dirac distribution at the mean value. Thermal transport at room temperature is well within the linear response regime and, in many experiments currently performed in labs, the transport is dominated by elastic processes.

The Sommerfeld series for the $\mathcal{L}_{p \alpha}^{(\nu)}$ coefficients are as follows

$$
\mathcal{L}_{p \alpha}^{(0)}=\mathcal{T}_{p \alpha}\left(\mu_{0}\right)+2 \Theta(2) T_{0}^{2} \mathcal{T}_{p \alpha}^{(2)}+2 \Theta(4) T_{0}^{4} \mathcal{T}_{p \alpha}^{(4)}\left(\mu_{0}\right)+\ldots
$$

and

$$
\begin{aligned}
\mathcal{L}_{p \alpha}^{(1)}=4 \Theta(2) T_{0}^{2} \mathcal{T}_{p \alpha}^{(1)}\left(\mu_{0}\right) & +8 \Theta(4) T_{0}^{4} \mathcal{T}_{p \alpha}^{(3)}\left(\mu_{0}\right) \\
& +12 \Theta(6) T_{0}^{6} \mathcal{T}_{p \alpha}^{(5)}\left(\mu_{0}\right)+\ldots
\end{aligned}
$$

and

$$
\begin{aligned}
\mathcal{L}_{p \alpha}^{(2)}=4 \Theta(2) T_{0}^{2} \mathcal{T}_{p \alpha}\left(\mu_{0}\right) & +24 \Theta(4) T_{0}^{4} \mathcal{T}_{p \alpha}^{(2)}\left(\mu_{0}\right) \\
& +60 \Theta(6) T_{0}^{6} \mathcal{T}_{p \alpha}^{(4)}\left(\mu_{0}\right)+\ldots,
\end{aligned}
$$

where $\Theta$ is a numerical factor and relates to the Riemann-Zeta function (here we've used the notation of Ref. [48]):

$$
\Theta(k+1)=\left(1-\frac{1}{2^{k}}\right) \zeta(k+1)
$$


explicitly:

$$
\begin{aligned}
\Theta(2) & =\frac{\pi^{2}}{12} \\
\Theta(4) & =\left(\frac{7}{8}\right) \frac{\pi^{4}}{90} \\
\Theta(6) & =\left(\frac{31}{32}\right) \frac{\pi^{6}}{945} .
\end{aligned}
$$

The transmission function has appreciable changes on an energy scale determined by the system's Hamiltonian and its couplings to the interconnect. We thus define the characteristic energy scale $\Delta$

$$
\mathcal{T}_{p \alpha}=\Delta^{2} \mathcal{T}_{p \alpha}^{(2)}
$$

which is typically much larger than the thermal energy $k_{B} T_{0}$.

The following relation connecting $\mathcal{L}_{p \alpha}^{(2)}$ and $\mathcal{L}_{p \alpha}^{(0)}$ is the Wiedemann-Franz law:

$$
\mathcal{L}_{p \alpha}^{(2)}=\frac{\pi^{2}}{3} k_{B}^{2} T_{0}^{2} \mathcal{L}_{p \alpha}^{(0)}\left(1+\mathcal{O}\left(\left(k_{B} T_{0} / \Delta\right)^{2}\right)\right)
$$




\section{REFERENCES}

[1] J Casas-Vázquez and D Jou. Temperature in non-equilibrium states: a review of open problems and current proposals. Reports on Progress in Physics, 66 (11):1937, 2003. URL http://stacks .iop.org/0034-4885/66/i=11/a=R03.

[2] R. Fowler and E.A. Guggenheim. Statistical Thermodynamics. Cambridge University Press, 1939.

[3] R. Clausius. Ueber eine veränderte form des zweiten hauptsatzes der mechanischen wärmetheorie. Annalen der Physik, 169(12):481-506, 1854. ISSN 1521-3889. doi: 10.1002/andp.18541691202. URL http://dx.doi.org/10. 1002/andp. 18541691202.

[4] W. Thomson. On an absolute thermometric scale founded on carnot's theory of the motive power of heat, and calculated from regnault's observations. Math. and Phys. Papers, pages 100-106, 1848.

[5] F. Reif. Fundamentals of Statistical and Thermal Physics. McGraw-Hill, 1965.

[6] S.R. De Groot and P. Mazur. Non-equilibrium Thermodynamics. NorthHolland, Amsterdam, 1962.

[7] P. Glansdorff and I. Prigogine. Thermodynamic Theory of Structure, Stability and Fluctuations. Wiley, 1971.

[8] G. E. Moore. Cramming more components onto integrated circuits. Electronics, 38(8), 1965.

[9] S. Datta. Electronic Transport in Mesoscopic Systems. Cambridge University Press, Cambridge, UK, 1995.

[10] B. J. van Wees, H. van Houten, C. W. J. Beenakker, J. G. Williamson, L. P. Kouwenhoven, D. van der Marel, and C. T. Foxon. Quantized conductance of point contacts in a two-dimensional electron gas. Phys. Rev. Lett., 60:848-850, Feb 1988. doi: 10.1103/PhysRevLett.60.848. URL https://link.aps.org/ doi/10.1103/PhysRevLett.60.848.

[11] K. v. Klitzing, G. Dorda, and M. Pepper. New method for high-accuracy determination of the fine-structure constant based on quantized hall resistance. Phys. Rev. Lett., 45:494-497, Aug 1980. doi: 10.1103/PhysRevLett.45.494. URL https://link.aps.org/doi/10.1103/PhysRevLett.45.494 
[12] S. Washburn and R. A. Webb. Aharonov-bohm effect in normal metal quantum coherence and transport. Adv. Phys., 35:375-422, 1986.

[13] G. Binnig, H. Rohrer, Ch. Gerber, and E. Weibel. Tunneling through a controllable vacuum gap. Applied Physics Letters, 40(2):178-180, 1982. doi: http://dx.doi.org/10.1063/1.92999. URL http://scitation.aip.org/ content/aip/journal/apl/40/2/10.1063/1.92999.

[14] G. Binnig and H. Rohrer. Scanning tunneling microscopy. Surface Science, 126(1):236 - 244, 1983. ISSN 0039-6028. doi: http://dx.doi. org/10.1016/0039-6028(83)90716-1. URL http://www.sciencedirect.com/ science/article/pii/0039602883907161.

[15] G. Binnig, C. F. Quate, and Ch. Gerber. Atomic force microscope. Phys. Rev. Lett., 56:930-933, Mar 1986. doi: 10.1103/PhysRevLett.56.930. URL http://link.aps.org/doi/10.1103/PhysRevLett.56.930.

[16] C. Julian Chen. Introduction to Scanning Tunneling Microscopy. Oxford University Press, New York, 2nd edition, 1993.

[17] C. C. Williams and H. K. Wickramasinghe. Scanning thermal profiler. Applied Physics Letters, 49(23):1587-1589, 1986. doi: http://dx.doi.org/10.1063/ 1.97288. URL http://scitation.aip.org/content/aip/journal/apl/49/ 23/10.1063/1.97288.

[18] P. Muralt and D. W. Pohl. Scanning tunneling potentiometry. Applied Physics Letters, 48(8):514-516, 1986. doi: http://dx.doi.org/10.1063/1. 96491. URL http://scitation.aip.org/content/aip/journal/apl/48/ 8/10.1063/1.96491.

[19] J. C. Cuevas and Elke Scheer. Molecular Electronics: An Introduction to Theory and Experiment. World Scientific, 2010.

[20] J. K. Gimzewski and R. Möller. Transition from the tunneling regime to point contact studied using scanning tunneling microscopy. Phys. Rev. B, 36:12841287, Jul 1987. doi: 10.1103/PhysRevB.36.1284. URL https://link.aps. org/doi/10.1103/PhysRevB.36.1284.

[21] Jonathan R. Widawsky, Pierre Darancet, Jeffrey B. Neaton, and Latha Venkataraman. Simultaneous determination of conductance and thermopower of single molecule junctions. Nano Letters, 12(1):354-358, 2012. doi: 10.1021/nl203634m. URL http://dx.doi.org/10.1021/nl203634m. PMID: 22128800 . 
[22] Charalambos Evangeli, Manuel Matt, Laura Rincn-Garca, Fabian Pauly, Peter Nielaba, Gabino Rubio-Bollinger, Juan Carlos Cuevas, and Nicols Agrat. Quantum thermopower of metallic atomic-size contacts at room temperature. Nano Letters, 15(2):1006-1011, 2015. doi: 10.1021/nl503853v. URL http://dx.doi.org/10.1021/n1503853v, PMID: 25607343.

[23] N. Agraït, J. G. Rodrigo, and S. Vieira. Conductance steps and quantization in atomic-size contacts. Phys. Rev. B, 47:12345-12348, 1993.

[24] Elke Scheer, Nicolás Agraït, Juan Carlos Cuevas, Alfredo Levy Yeyati, Bas Ludoph, Alvaro Martín-Rodero, Gabino Rubio Bollinger, Jan M. van Ruitenbeek, and Cristián Urbina. The signature of chemical valence in the electrical conduction through a single-atom contact. Nature, 394:154 EP -, Jul 1998. URL http://dx .doi .org/10.1038/28112.

[25] N. Agraït, A. Levy Yeyati, and J. M. van Ruitenbeek. Quantum properties of atomic-sized conductors. Phys. Rep., 377:81-279, 2003, and references therein.

[26] Woochul Lee, Kyeongtae Kim, Wonho Jeong, Linda Angela Zotti, Fabian Pauly, Juan Carlos Cuevas, and Pramod Reddy. Heat dissipation in atomicscale junctions. Nature, 498(7453):209-212, June 2013. ISSN 0028-0836. URL http://dx.doi.org/10.1038/nature12183.

[27] Longji Cui, Wonho Jeong, Sunghoon Hur, Manuel Matt, Jan C. Klöckner, Fabian Pauly, Peter Nielaba, Juan Carlos Cuevas, Edgar Meyhofer, and Pramod Reddy. Quantized thermal transport in single-atom junctions. Science, 355(6330):1192-1195, 2017. ISSN 0036-8075. doi: 10.1126/ science.aam6622. URL http://science.sciencemag.org/content/355/ $6330 / 1192$.

[28] Nico Mosso, Ute Drechsler, Fabian Menges, Peter Nirmalraj, Siegfried Karg, Heike Riel, and Bernd Gotsmann. Heat transport through atomic contacts. Nat Nano, 12(5):430-433, May 2017. ISSN 1748-3387. URL http://dx.doi. org/10.1038/nnano.2016.302. Letter.

[29] J.A. McLennan. Introduction to Non-equilibrium Statistical Mechanics. Prentice Hall, 1989.

[30] J. Meair, J. P. Bergfield, C. A. Stafford, and Ph. Jacquod. Local temperature of out-of-equilibrium quantum electron systems. Phys. Rev. B, 90:035407, Jul 2014. doi: 10.1103/PhysRevB.90.035407. URL http://link.aps.org/doi/ 10.1103/PhysRevB.90.035407. 
[31] Charles A. Stafford. Local temperature of an interacting quantum system far from equilibrium. Phys. Rev. B, 93:245403, Jun 2016. doi: 10.1103/PhysRevB. 93.245403. URL http://link.aps.org/doi/10.1103/PhysRevB.93.245403.

[32] Lars Onsager. Reciprocal relations in irreversible processes. i. Phys. Rev., 37: 405-426, Feb 1931. doi: 10.1103/PhysRev.37.405. URL http://link.aps. org/doi/10.1103/PhysRev.37.405.

[33] Lars Onsager. Reciprocal relations in irreversible processes. ii. Phys. Rev., 38: 2265-2279, Dec 1931. doi: 10.1103/PhysRev.38.2265. URL https://link. aps.org/doi/10.1103/PhysRev.38.2265.

[34] Paul C. Martin and Julian Schwinger. Theory of many-particle systems. i. Phys. Rev., 115:1342-1373, Sep 1959. doi: 10.1103/PhysRev.115.1342. URL https://link.aps.org/doi/10.1103/PhysRev.115.1342.

[35] Julian Schwinger. Brownian motion of a quantum oscillator. Journal of Mathematical Physics, 2(3):407-432, 1961. doi: 10.1063/1.1703727. URL https://doi.org/10.1063/1.1703727.

[36] L. P. Kadanoff and G. Baym. Quantum Statistical Mechanics. Addison Wesley, 1994.

[37] L. V. Keldysh. Diagram technique for nonequilibrium processes. Zh. Eksp. Teor. Fiz., 47:1515-1527, 1964.

[38] Y. Meir and N. S. Wingreen. Landauer formula for the current through an interacting electron region. Phys. Rev. Lett., 68:2512-2515, 1992.

[39] Gianluca Stefanucci and Robert van Leeuwen. Nonequilibrium Many-Body Theory Of Quantum Systems: A Modern Introduction. Cambridge University Press, 2013.

[40] Joshua Barr. Transport in interacting nanostructures, 2013. URL http: //hdl.handle.net/10150/301551. PhD Thesis, University of Arizona.

[41] Abhay Shastry and Charles A. Stafford. Temperature and voltage measurement in quantum systems far from equilibrium. Phys. Rev. B, 94:155433, Oct 2016. doi: 10.1103/PhysRevB.94.155433. URL http://link.aps.org/doi/ 10.1103/PhysRevB.94.155433.

[42] Arthur Stanley Eddington. The nature of the physical world. The University Press, Cambridge, England, 1929. 
[43] David Ruelle. Natural nonequilibrium states in quantum statistical mechanics. Journal of Statistical Physics, 98(1):57-75, 2000. doi: 10.1023/A: 1018618704438. URL http://dx.doi.org/10.1023/A:1018618704438.

[44] Georgy Lebon and David Jou. Understanding Non-equilibrium Thermodynamics. Springer-Verlag Berlin Heidelberg, 2008. doi: 10.1007/978-3-540-74252-4.

[45] Leticia F Cugliandolo. The effective temperature. Journal of Physics A: Mathematical and Theoretical, 44(48):483001, 2011. URL http://stacks. iop.org/1751-8121/44/i=48/a=483001.

[46] Ph. A. Jacquet and C.-A. Pillet. Temperature and voltage probes far from equilibrium. Phys. Rev. B, 85:125120, Mar 2012. doi: 10.1103/PhysRevB.85. 125120. URL http://link.aps.org/doi/10.1103/PhysRevB.85.125120.

[47] Massimiliano Esposito, Maicol A. Ochoa, and Michael Galperin. Quantum thermodynamics: A nonequilibrium green's function approach. Phys. Rev. Lett., 114:080602, Feb 2015. doi: 10.1103/PhysRevLett.114.080602. URL http://link.aps.org/doi/10.1103/PhysRevLett.114.080602.

[48] Abhay Shastry and Charles A. Stafford. Cold spots in quantum systems far from equilibrium: Local entropies and temperatures near absolute zero. Phys. Rev. B, 92:245417, Dec 2015. doi: 10.1103/PhysRevB.92.245417. URL http: //link.aps.org/doi/10.1103/PhysRevB.92.245417.

[49] Taizo Kanagawa, Rei Hobara, Iwao Matsuda, Takehiro Tanikawa, Akiko Natori, and Shuji Hasegawa. Anisotropy in conductance of a quasione-dimensional metallic surface state measured by a square micro-fourpoint probe method. Phys. Rev. Lett., 91:036805, Jul 2003. doi: 10. 1103/PhysRevLett.91.036805. URL http://link.aps.org/doi/10.1103/ PhysRevLett.91.036805.

[50] A. Bannani, C. A. Bobisch, and R. Moeller. Local potentiometry using a multiprobe scanning tunneling microscope. Review Of Scientific Instruments, 79(8), aug 2008. ISSN 0034-6748. doi: $\{10.1063 / 1.2968111\}$.

[51] F. Luepke, S. Korte, V. Cherepanov, and B. Voigtlaender. Scanning tunneling potentiometry implemented into a multi-tip setup by software. Review Of Scientific Instruments, 86(12), dec 2015. ISSN 0034-6748. doi: \{10.1063/1. 4936079\}.

[52] Kyeongtae Kim, Jaehun Chung, Gwangseok Hwang, Ohmyoung Kwon, and Joon Sik Lee. Quantitative measurement with scanning thermal microscope by preventing the distortion due to the heat transfer through the air. $A C S$ 
Nano, 5(11):8700-8709, 2011. doi: 10.1021/nn2026325. URL http://dx.doi. org/10.1021/nn2026325, PMID: 21999681.

[53] Young-Jun Yu, Melinda Y. Han, Stphane Berciaud, Alexandru B. Georgescu, Tony F. Heinz, Louis E. Brus, Kwang S. Kim, and Philip Kim. Highresolution spatial mapping of the temperature distribution of a joule selfheated graphene nanoribbon. Applied Physics Letters, 99(18):183105, 2011. doi: http://dx.doi.org/10.1063/1.3657515. URL http://scitation.aip. org/content/aip/journal/apl/99/18/10.1063/1.3657515.

[54] Kyeongtae Kim, Wonho Jeong, Woochul Lee, and Pramod Reddy. Ultra-high vacuum scanning thermal microscopy for nanometer resolution quantitative thermometry. ACS Nano, 6(5):4248-4257, 2012. doi: 10.1021/nn300774n. URL http://dx.doi .org/10.1021/nn300774n. PMID: 22530657.

[55] Fabian Menges, Heike Riel, Andreas Stemmer, and Bernd Gotsmann. Quantitative thermometry of nanoscale hot spots. Nano Letters, 12(2):596-601, 2012. doi: 10.1021/nl203169t. URL http://dx.doi.org/10.1021/nl203169t. PMID: 22214277.

[56] H.-L. Engquist and P. W. Anderson. Definition and measurement of the electrical and thermal resistances. Phys. Rev. B, 24:1151-1154, Jul 1981. doi: 10.1103/PhysRevB.24.1151. URL http://link .aps.org/doi/10.1103/ PhysRevB.24.1151.

[57] Yonatan Dubi and Massimiliano Di Ventra. Thermoelectric effects in nanoscale junctions. Nano Letters, 9:97-101, 2009.

[58] Philippe A. Jacquet. Thermoelectric transport properties of a chain ofquantum dots with self-consistent reservoirs. Journal of Statistical Physics, 134(4):709748, 2009. ISSN 1572-9613. doi: 10.1007/s10955-009-9697-1. URL http: //dx.doi.org/10.1007/s10955-009-9697-1.

[59] Y. Dubi and M. Di Ventra. Fourier's law: Insight from a simple derivation. Phys. Rev. E, 79:042101, Apr 2009. doi: 10.1103/PhysRevE.79.042101. URL http://link.aps.org/doi/10.1103/PhysRevE.79.042101.

[60] Alvaro Caso, Liliana Arrachea, and Gustavo S. Lozano. Local temperatures and heat flow in quantum driven systems. Phys. Rev. B, 83:165419, Apr 2011. doi: 10.1103/PhysRevB.83.165419. URL http://link.aps.org/doi/ 10.1103/PhysRevB.83.165419.

[61] David Sánchez and Llorenç Serra. Thermoelectric transport of mesoscopic conductors coupled to voltage and thermal probes. Phys. Rev. B, 84:201307, 
Nov 2011. doi: 10.1103/PhysRevB.84.201307. URL http://link.aps.org/ doi/10.1103/PhysRevB.84.201307.

[62] Alvaro Caso, Liliana Arrachea, and Gustavo S. Lozano. Local and effective temperatures of quantum driven systems. Phys. Rev. B, 81(4):041301, Jan 2010. doi: 10.1103/PhysRevB.81.041301.

[63] Justin P. Bergfield, Shauna M. Story, Robert C. Stafford, and Charles A. Stafford. Probing maxwell's demon with a nanoscale thermometer. ACS Nano, 7(5):4429-4440, 2013. doi: 10.1021/nn401027u.

[64] Justin P. Bergfield, Mark A. Ratner, Charles A. Stafford, and Massimiliano Di Ventra. Tunable quantum temperature oscillations in graphene nanostructures. Phys. Rev. B, 91:125407, Mar 2015. doi: 10.1103/PhysRevB.91.125407. URL http://link.aps .org/doi/10.1103/PhysRevB.91.125407.

[65] LvZhou Ye, Dong Hou, Xiao Zheng, YiJing Yan, and Massimiliano Di Ventra. Local temperatures of strongly-correlated quantum dots out of equilibrium. Phys. Rev. B, 91:205106, May 2015. doi: 10.1103/PhysRevB.91.205106. URL http://link.aps.org/doi/10.1103/PhysRevB.91.205106.

[66] Y.C. Chen, M. Zwolak, and M. Di Ventra. Local heating in nanoscale conductors. Nano Lett., 3:1691, 2003.

[67] Yi Ming, Zhe Xian Wang, Ze Jun Ding, and Hui Min Li. Ballistic thermal rectification in asymmetric three-terminal mesoscopic dielectric systems. New Journal of Physics, 12:103041, 2010.

[68] Michael Galperin, Abraham Nitzan, and Mark A. Ratner. Heat conduction in molecular transport junctions. Phys. Rev. B, 75:155312, Apr 2007. doi: 10.1103/PhysRevB.75.155312. URL http://link.aps.org/doi/10.1103/ PhysRevB.75.155312.

[69] Y. de Wilde, F. Formanek, R. Carminati, B. Gralak, P.-A. Lemoine, K. Joulain, J.-P. Mulet, Y. Chen, and J.-J. Greffet. Thermal radiation scanning tunnelling microscopy. Nature, 444:740-743, December 2006. doi: $10.1038 /$ nature05265.

[70] Yanan Yue, Jingchao Zhang, and Xinwei Wang. Micro/nanoscale spatial resolution temperature probing for the interfacial thermal characterization of epitaxial graphene on 4h-sic. Small, 7(23):3324-3333, 2011. ISSN 1613-6829.

[71] Jean-Jacques Greffet and Carsten Henkel. Coherent thermal radiation. Contemp. Phys., 48(4):183-194, 2007. doi: 10.1080/00107510701690380. 
[72] J. P. Bergfield and C. A. Stafford. Thermoelectric signatures of coherent transport in single-molecule heterojunctions. Nano Letters, 9:3072-3076, 2009.

[73] Roger A. Horn and Charles R. Johnson, editors. Matrix Analysis. Cambridge University Press, New York, NY, USA, 1986. ISBN 0-521-30586-1.

[74] Justin P. Bergfield and Charles A. Stafford. Thermoelectric corrections to quantum voltage measurement. Phys. Rev. B, 90:235438, Dec 2014. doi: 10.1103/PhysRevB.90.235438. URL http://link.aps.org/doi/10.1103/ PhysRevB.90.235438.

[75] Sergei V. Kalinin and Alexei Gruverman. Scanning Probe Microscopy: Electrical and Electromechanical Phenomena at the Nanoscale. Springer-Verlag, 2007. ISBN 978-0-387-28667-9. doi: 10.1007/978-0-387-28668-6.

[76] H. Pothier, S. Guéron, Norman O. Birge, D. Esteve, and M. H. Devoret. Energy distribution function of quasiparticles in mesoscopic wires. Phys. Rev. Lett., 79:3490-3493, Nov 1997. doi: 10.1103/PhysRevLett.79.3490. URL https://link.aps.org/doi/10.1103/PhysRevLett.79.3490.

[77] U. Sivan and Y. Imry. Multichannel landauer formula for thermoelectric transport with application to thermopower near the mobility edge. Phys. Rev. B, 33:551-558, Jan 1986. doi: 10.1103/PhysRevB.33.551. URL http: //link.aps.org/doi/10.1103/PhysRevB.33.551.

[78] M. Büttiker. Coherent and sequential tunneling in series barriers. IBM J. Res. Dev., 32:63, 1988.

[79] M. Büttiker. Chemical potential oscillations near a barrier in the presence of transport. Phys. Rev. B, 40(5):3409-3412, Aug 1989. doi: 10.1103/PhysRevB. 40.3409 .

[80] A. D. Benoit, S. Washburn, C. P. Umbach, R. B. Laibowitz, and R. A. Webb. Asymmetry in the magnetoconductance of metal wires and loops. Phys. Rev. Lett., 57:1765-1768, Oct 1986. doi: 10.1103/PhysRevLett.57.1765. URL http: //link.aps.org/doi/10.1103/PhysRevLett.57.1765.

[81] K. L. Shepard, M. L. Roukes, and B. P. van der Gaag. Experimental measurement of scattering coefficients in mesoscopic conductors. Phys. Rev. B, 46:9648-9666, Oct 1992. doi: 10.1103/PhysRevB.46.9648. URL http: //link.aps.org/doi/10.1103/PhysRevB.46.9648.

[82] R. de Picciotto, H. L. Stormer, L. N. Pfeiffer, K. W. Baldwin, and K. W. West. Four-terminal resistance of a ballistic quantum wire. Nature, 411(6833):51-54, May 2001. ISSN 0028-0836. URL http://dx.doi.org/10.1038/35075009. 
[83] B. Gao, Y. F. Chen, M. S. Fuhrer, D. C. Glattli, and A. Bachtold. Four-point resistance of individual single-wall carbon nanotubes. Phys. Rev. Lett., 95: 196802, Oct 2005. doi: 10.1103/PhysRevLett.95.196802. URL http://link. aps.org/doi/10.1103/PhysRevLett.95.196802.

[84] M. Büttiker. Small normal-metal loop coupled to an electron reservoir. Phys. Rev. B, 32:1846-1849, Aug 1985. doi: 10.1103/PhysRevB.32.1846. URL http: //link.aps.org/doi/10.1103/PhysRevB.32.1846.

[85] M. Büttiker. Role of quantum coherence in series resistors. Phys. Rev. B, 33: 3020-3026, Mar 1986. doi: 10.1103/PhysRevB.33.3020. URL http://link. aps.org/doi/10.1103/PhysRevB.33.3020.

[86] Jorge L. D'Amato and Horacio M. Pastawski. Conductance of a disordered linear chain including inelastic scattering events. Phys. Rev. B, 41:7411-7420, Apr 1990. doi: 10.1103/PhysRevB.41.7411. URL http://link.aps.org/ doi/10.1103/PhysRevB.41.7411.

[87] Tsuneya Ando. Voltage distribution and phase-breaking scattering in the quantum hall regime. Surface Science, 361362:270 - 273, 1996. ISSN 00396028. doi: http://dx.doi.org/10.1016/0039-6028(96)00400-1. URL http:// wWw.sciencedirect.com/science/article/pii/0039602896004001. Proceedings of the Eleventh International Conference on the Electronic Properties of Two-Dimensional Systems.

[88] Dibyendu Roy and Abhishek Dhar. Electron transport in a one dimensional conductor with inelastic scattering by self-consistent reservoirs. Phys. Rev. B, 75:195110, May 2007. doi: 10.1103/PhysRevB.75.195110. URL http: //link.aps.org/doi/10.1103/PhysRevB.75.195110.

[89] M.J.M. de Jong and C.W.J. Beenakker. Semiclassical theory of shot noise in mesoscopic conductors. Physica A: Statistical Mechanics and its Applications, 230(1-2):219-248, August 1996. ISSN 0378-4371. URL http: //wWw.sciencedirect.com/science/article/pii/0378437196000684.

[90] S. A. van Langen and M. Büttiker. Quantum-statistical current correlations in multilead chaotic cavities. Phys. Rev. B, 56:R1680-R1683, Jul 1997. doi: 10.1103/PhysRevB.56.R1680. URL http://link.aps.org/doi/ 10.1103/PhysRevB.56.R1680.

[91] Heidi Förster, Peter Samuelsson, Sebastian Pilgram, and Markus Büttiker. Voltage and dephasing probes in mesoscopic conductors: A study of fullcounting statistics. Phys. Rev. B, 75:035340, Jan 2007. doi: 10.1103/ PhysRevB.75.035340. URL http://link.aps.org/doi/10.1103/PhysRevB . 75.035340 . 
[92] Keiji Saito, Giuliano Benenti, Giulio Casati, and Toma ž Prosen. Thermopower with broken time-reversal symmetry. Phys. Rev. B, 84:201306, Nov 2011. doi: 10.1103/PhysRevB.84.201306. URL http://link.aps.org/doi/ 10.1103/PhysRevB.84.201306.

[93] Vinitha Balachandran, Giuliano Benenti, and Giulio Casati. Efficiency of three-terminal thermoelectric transport under broken time-reversal symmetry. Phys. Rev. B, 87:165419, Apr 2013. doi: 10.1103/PhysRevB.87.165419. URL http://link.aps.org/doi/10.1103/PhysRevB.87.165419.

[94] Kay Brandner, Keiji Saito, and Udo Seifert. Strong bounds on onsager coefficients and efficiency for three-terminal thermoelectric transport in a magnetic field. Phys. Rev. Lett., 110:070603, Feb 2013. doi: 10.1103/PhysRevLett. 110.070603. URL http://link.aps.org/doi/10.1103/PhysRevLett.110. 070603.

[95] Malay Bandyopadhyay and Dvira Segal. Quantum heat transfer in harmonic chains with self-consistent reservoirs: Exact numerical simulations. Phys. Rev. E, 84:011151, Jul 2011. doi: 10.1103/PhysRevE.84.011151. URL http:// link.aps.org/doi/10.1103/PhysRevE.84.011151.

[96] Salil Bedkihal, Malay Bandyopadhyay, and Dvira Segal. The probe technique far from equilibrium: Magnetic field symmetries of nonlinear transport. The European Physical Journal B, 86(12), 2013. doi: 10.1140/epjb/e2013-40971-7.

[97] Salil Bedkihal, Malay Bandyopadhyay, and Dvira Segal. Magnetic field symmetries of nonlinear transport with elastic and inelastic scattering. Phys. Rev. B, 88:155407, Oct 2013. doi: 10.1103/PhysRevB.88.155407. URL http://link.aps.org/doi/10.1103/PhysRevB.88.155407.

[98] Justin P. Bergfield, Michelle A. Solis, and Charles A. Stafford. Giant thermoelectric effect from transmission supernodes. ACS Nano, 4(9):5314-5320, 2010 .

[99] RK Pathria and PD Beale. Statistical Mechanics, 3rd Edition. Elsevier Butterworth-Heinemann, 2011. ISBN 978-0-12-382189-8; 978-0-12-382188-1.

[100] Justin P. Bergfield, Mark A. Ratner, Charles A. Stafford, and Massimiliano Di Ventra. Tunable quantum temperature oscillations in graphene nanostructures. Phys. Rev. B, 91:125407, Mar 2015. doi: 10.1103/PhysRevB.91.125407. URL http://link .aps .org/doi/10.1103/PhysRevB.91.125407.

[101] J. K. Viljas, J. C. Cuevas, F. Pauly, and M. Häfner. Electron-vibration interaction in transport through atomic gold wires. Phys. Rev. B, 72(24), 2005. 
[102] M. Büttiker. 4-Terminal phase-coherent conductance. Phys. Rev. Lett., 57: $1761,1986$.

[103] J. Heurich, J. C. Cuevas, W. Wenzel, and G. Schön. Electrical transport through single-molecule junctions: From molecular orbitals to conduction channels. Phys. Rev. Lett., 88:256803, 2002.

[104] J. P. Bergfield and C. A. Stafford. Many-body theory of electronic transport in single-molecule heterojunctions. Phys. Rev. B, 79(24):245125, 2009.

[105] Yonatan Dubi and Massimiliano Di Ventra. Thermoelectric effects in nanoscale junctions. Nano Lett., 9(1):97-101, 2009.

[106] Hyunwook Song, Youngsang Kim, Yun Hee Jang, Heejun Jeong, Mark A. Reed, and Takhee Lee. Observation of molecular orbital gating. Nature, 462 (7276):1039-1043, December 2009. ISSN 0028-0836. URL http://dx.doi. org/10.1038/nature08639.

[107] Luis G. C. Rego and George Kirczenow. Quantized thermal conductance of dielectric quantum wires. prl, 81(1):232-235, Jul 1998.

[108] Luis G. C. Rego and George Kirczenow. Fractional exclusion statistics and the universal quantum of thermal conductance: A unifying approach. Phys. Rev. B, 59(20):13080-13086, 1999.

[109] G. Kucsko, P. C. Maurer, N. Y. Yao, M. Kubo, H. J. Noh, P. K. Lo, H. Park, and M. D. Lukin. Nanometre-scale thermometry in a living cell. Nature, 500(7460):54-58, Aug 2013. ISSN 0028-0836. URL http://dx.doi.org/10. 1038/nature12373. Letter.

[110] C. Yan Jin, Zhiyong Li, R. Stanley Williams, K.-Cheol Lee, and Inkyu Park. Localized temperature and chemical reaction control in nanoscale space by nanowire array. Nano Letters, 11(11):4818-4825, 2011. doi: 10.1021/nl2026585. URL http://dx.doi.org/10.1021/nl2026585. PMID: 21967343.

[111] Matthew Mecklenburg, William A. Hubbard, E. R. White, Rohan Dhall, Stephen B. Cronin, Shaul Aloni, and B. C. Regan. Nanoscale temperature mapping in operating microelectronic devices. Science, 347(6222):629632, 2015. ISSN 0036-8075. doi: 10.1126/science.aaa2433. URL http: //science.sciencemag.org/content/347/6222/629.

[112] J. S. Reparaz, E. Chavez-Angel, M. R. Wagner, B. Graczykowski, J. GomisBresco, F. Alzina, and C. M. Sotomayor Torres. A novel contactless technique for thermal field mapping and thermal conductivity determination: Two-laser 
raman thermometry. Review of Scientific Instruments, 85(3):034901, 2014. doi: 10.1063/1.4867166. URL http://dx.doi.org/10.1063/1.4867166.

[113] P. Neumann, I. Jakobi, F. Dolde, C. Burk, R. Reuter, G. Waldherr, J. Honert, T. Wolf, A. Brunner, J. H. Shim, D. Suter, H. Sumiya, J. Isoya, and J. Wrachtrup. High-precision nanoscale temperature sensing using single defects in diamond. Nano Letters, 13(6):2738-2742, 2013. doi: 10.1021/ nl401216y. URL http://dx.doi.org/10.1021/nl401216y. PMID: 23721106.

[114] D. Teyssieux, L. Thiery, and B. Cretin. Near-infrared thermography using a charge-coupled device camera: Application to microsystems. Review of Scientific Instruments, 78(3):034902, 2007. doi: 10.1063/1.2714040. URL http://dx.doi.org/10.1063/1.2714040.

[115] A Majumdar. Scanning thermal microscopy. Annual Review of Materials Science, 29:505-585, 1999. ISSN 0084-6600. doi: 10.1146/annurev.matsci.29. 1.505 .

[116] Wonho Jeong, Sunghoon Hur, Edgar Meyhofer, and Pramod Reddy. Scanning probe microscopy for thermal transport measurements. Nanoscale and Microscale Thermophysical Engineering, 19(4):279-302, 2015. doi: 10.1080/ 15567265.2015.1109740. URL http://dx.doi.org/10.1080/15567265. 2015.1109740.

[117] Fabian Menges, Philipp Mensch, Heinz Schmid, Heike Riel, Andreas Stemmer, and Bernd Gotsmann. Temperature mapping of operating nanoscale devices by scanning probe thermometry. Nature Communications, 7:10874 EP -, Mar 2016. URL http://dx.doi.org/10.1038/ncomms10874. Article.

[118] B. G. Briner, R. M. Feenstra, T. P. Chin, and J. M. Woodall. Local transport properties of thin bismuth films studied by scanning tunneling potentiometry. Phys. Rev. B, 54:R5283-R5286, Aug 1996. doi: 10.1103/PhysRevB.54.R5283. URL https://link.aps .org/doi/10.1103/PhysRevB.54.R5283.

[119] Geetha Ramaswamy and A. K. Raychaudhuri. Field and potential around local scatterers in thin metal films studied by scanning tunneling potentiometry. Applied Physics Letters, 75(13):1982-1984, 1999. doi: 10.1063/1.124892. URL http://dx.doi.org/10.1063/1.124892.

[120] Weigang Wang, Ko Munakata, Michael Rozler, and Malcolm R. Beasley. Local Transport Measurements at Mesoscopic Length Scales Using Scanning Tunneling Potentiometry. Physical Review Letters, 110(23), jun 2013. ISSN 00319007. doi: \{10.1103/PhysRevLett.110.236802\}. 
[121] Kendal W. Clark, X.-G. Zhang, Gong Gu, Jewook Park, Guowei He, R. M. Feenstra, and An-Ping Li. Energy gap induced by friedel oscillations manifested as transport asymmetry at monolayer-bilayer graphene boundaries. Phys. Rev. X, 4:011021, Feb 2014. doi: 10.1103/PhysRevX.4.011021. URL http://link.aps.org/doi/10.1103/PhysRevX.4.011021.

[122] Philip Willke, Thomas Druga, Rainer G. Ulbrich, M. Alexander Schneider, and Martin Wenderoth. Spatial extent of a landauer residual-resistivity dipole in graphene quantified by scanning tunnelling potentiometry. Nature Communications, 6:-, March 2015. URL http://dx.doi.org/10.1038/ncomms7399.

[123] R. Landauer. Spatial variation of currents and fields due to localized scatterers in metallic conduction. IBM Journal of Research and Development, 1(3):223231, July 1957. ISSN 0018-8646. doi: 10.1147/rd.13.0223.

[124] Séverine Gomès, Ali Assy, and Pierre-Olivier Chapuis. Scanning thermal microscopy: A review. physica status solidi (a), 212(3):477-494, 2015. ISSN 18626319. doi: 10.1002/pssa.201400360. URL http://dx.doi.org/10.1002/ pssa.201400360.

[125] Makusu Tsutsui, Tomoji Kawai, and Masateru Taniguchi. Unsymmetrical hot electron heating in quasi-ballistic nanocontacts. Scientific Reports, 2, Jan 2012. URL http://dx.doi.org/10.1038/srep00217.

[126] Charles A. Stafford and Abhay Shastry. Local entropy of a nonequilibrium fermion system. The Journal of Chemical Physics, 146(9):092324, 2017. doi: 10.1063/1.4975810. URL http://dx.doi.org/10.1063/1.4975810.

[127] John von Neumann. Mathematical Foundations of Quantum Mechanics. Princeton Landmarks in Mathematics and Physics. Princeton University Press, Princeton, New Jersey, 1996. Translation from German edition (October 28, 1996).

[128] L. D. Landau and E. M. Lifshitz. Statistical Physics, pages 160-161. Butterworth-Heinemann, 3rd edition, 1980.

[129] Michael A. Nielsen and Isaac L. Chuang. Quantum Computation and Quantum Information: 10th Anniversary Edition. Cambridge University Press, New York, NY, USA, 10th edition, 2011. ISBN 1107002176, 9781107002173.

[130] V. Gasparian, T. Christen, and M. Büttiker. Partial densities of states, scattering matrices, and greens functions. Phys. Rev. A, 54:4022, 1996.

[131] H. Ness. Nonequilibrium distribution functions for quantum transport: Universality and approximation for the steady state regime. Phys. Rev. B, 89: 
045409, Jan 2014. doi: 10.1103/PhysRevB.89.045409. URL http://link. aps.org/doi/10.1103/PhysRevB.89.045409.

[132] J. L. W. V. Jensen. Sur les fonctions convexes et les inégalités entre les valeurs moyennes. Acta Mathematica, 30(1):175-193, 1906. ISSN 1871-2509. doi: 10.1007/BF02418571. URL http://dx.doi.org/10.1007/BF02418571.

[133] H. P. Breuer and F. Petruccione. The theory of open quantum systems. Oxford University Press, 2002.

[134] J. Gemmer, M. Michel, and G. Mahler. Quantum Thermodynamics: Emergence of Thermodynamic Behavior Within Composite Quantum Systems. Springer, 2009. doi: 10.1007/978-3-540-70510-9.

[135] Gilad Gour, Markus P. Mller, Varun Narasimhachar, Robert W. Spekkens, and Nicole Yunger Halpern. The resource theory of informational nonequilibrium in thermodynamics. Physics Reports, 583:1 - 58, 2015. ISSN 03701573. doi: http://dx.doi.org/10.1016/j.physrep.2015.04.003. URL http: //wWw.sciencedirect.com/science/article/pii/S037015731500229X.

[136] K. Micadei, J.P.S. Peterson, A.M. Souza, R.S. Sarthour, I.S. Oliveria, G.T. Landi, T.B. Batalhao, R.M. Serra, and E. Lutz. Reversing the thermodynamic arrow of time using quantum correlations. arXiv eprints, Nov 2017. doi: arXiv:1711.0332. URL https://arxiv.org/abs/1711.03323.

[137] Udo Seifert. Stochastic thermodynamics, fluctuation theorems and molecular machines. Reports on Progress in Physics, 75(12):126001, 2012. URL http: //stacks . iop.org/0034-4885/75/i=12/a=126001.

[138] Cyril Elouard, David A. Herrera-Martí, Maxime Clusel, and Alexia Auffèves. The role of quantum measurement in stochastic thermodynamics. $n p j$ Quantum Information, 3(1):9, 2017. ISSN 2056-6387. doi: 10.1038/ s41534-017-0008-4. URL https://doi .org/10.1038/s41534-017-0008-4. 